\title{
Power Minimization in Wireless Network Virtualization with Massive MIMO
}

by

Mohammadmoein Soltanizadeh

A thesis submitted in conformity with the requirements for the degree of Master of Applied Science Graduate Department of Electrical and Computer Engineering University of Toronto

(C) Copyright 2017 by Mohammadmoein Soltanizadeh 


\author{
Abstract \\ Power Minimization in Wireless Network Virtualization with Massive MIMO \\ Mohammadmoein Soltanizadeh \\ Master of Applied Science \\ Graduate Department of Electrical and Computer Engineering \\ University of Toronto \\ 2017
}

This thesis aims to study wireless network virtualization and particularly its challenges when it is applied to massive MIMO technology. Virtualization has been well studied in the wired networks and computational resources. However, when it comes to wireless networks new challenges arise. We consider virtualization in the context of massive MIMO technology and develop a novel precoding schemes to abstract and share a massive MIMO base station with the objective of minimizing power. This problem is investigated when perfect channel information is available and when is not. Furthermore, we show that our formulation is general enough to be easily extended to multi-cell and address some concerns of the service provider. We obtain optimal precoding when perfect channel information is available and a nearly optimal solution when the channel information is corrupted. 


\section{Acknowledgements}

First and foremost, thank you Professor Ben Liang for the mentorship, support and your kindness as my supervisor. I will never forget the time I was struggling with a problem but you kept me motivated to push my project forward. Your supervisory style allowed me to drive the research direction and grow as an independent person. Thanks for your endless help.

I would like to thank Dr. Gary Boudreau and Dr. Hossein Seyedmehdi for their advice and guidance. The first idea of this project inspired by a question you raised, and your valuable comments dramatically improved the quality of this work.

I would like to thank members of my committee, Professor A. Leon-Garcia, Professor A. Khisti and Professor A. Veneris for their valuable comments and interest in my work.

My wonderful friends dramatically improved this two years experience. They were nothing but fun. Their names list goes very long and I do not know in what order I should put the names. I thank you all, pals.

Finally, I would like to thank my parents, brother, and sister. You were always there for me and did the bests to me. 


\section{Contents}

$\begin{array}{ll}\text { Acknowledgements } & \text { iii }\end{array}$

Table of Contents $\quad$ iv

List of Tables $\quad$ vi

List of Figures $\quad$ vii

List of Algorithms $\quad$ ix

1 Introduction $\quad 1$

1.1 Wireless Network Virtualization: Promises and Challenges . . . . . . . . 1

1.2 Massive MIMO . . . . . . . . . . . . . . . . . . . 8

1.3 Literature Review . . . . . . . . . . . . . . . . . . . . 10

1.4 Thesis Outline . . . . . . . . . . . . . . . . . 13

2 System Model and Problem Statement 14

2.1 System Model . . . . . . . . . . . . . . . . . . . . . . 14

2.2 Power Minimization with Perfect CSI . . . . . . . . . . . . . . 17

2.2.1 Problem Statement . . . . . . . . . . . . . . . . . . . 17

2.2.2 Proposed Solution for Perfect CSI . . . . . . . . . . . . . . . . . 19

2.3 Power Minimization for Non-perfect CSI . . . . . . . . . . . . . . . . . . 21

2.3.1 Problem Statement . . . . . . . . . . . . . . . . . . 21

2.3.2 Proposed Solution for Non-Perfect CSI . . . . . . . . . . . . . . . 22

2.4 Three Extensions . . . . . . . . . . . . . . . . . . . . . . 26

2.4.1 Adding SINR constraints . . . . . . . . . . . 26

2.4 .2 Mitigating Inter-cell Interference . . . . . . . . . . . . . . . . . . . 27

2.4.3 Per User Constraint . . . . . . . . . . . . . . . . 27

2.5 Antenna Selection . . . . . . . . . . . . . . . . . 28 
2.5.1 Antenna Selection in Step $1 \ldots \ldots$. . . . . . . . . . 28

2.5 .2 Antenna Selection in Step $3 \ldots \ldots$. . . . . . . . . . 29

3 Simulations and Performance Evaluation $\quad 30$

3.1 Simulation Settings . . . . . . . . . . . . . . . . . . . . 30

3.2 Numerical Results for Precoding with Perfect CSI . . . . . . . . . . . 32

3.3 Numerical Results for Precoding with Imperfect CSI Precoding . . . . . . 33

3.3.1 Channel Estimation through Uplink Training . . . . . . . . . 33

3.3 .2 Results for Imperfect CSI _ . . . . . . . . . . . . . . . 37

3.4 Three Extensions . . . . . . . . . . . . . . . . . . . . . . . . 40

3.5 Antenna Selection Algorithms . . . . . . . . . . . . . . . . 50

4 Conclusion $\quad 55$

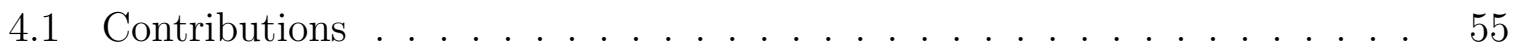

4.2 Future Research Direction . . . . . . . . . . . . . . . . . . 56

$\begin{array}{ll}\text { A Proofs } & 57\end{array}$

A.1 Proof of Lemma $2.2 .1 \ldots \ldots \ldots \ldots \ldots \ldots$

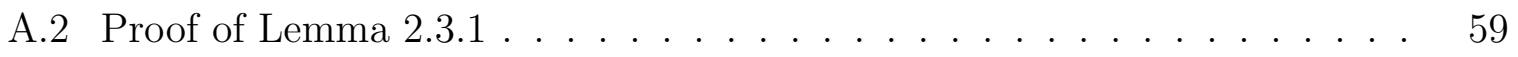

A.3 Transforming (2.11) to sum of random variables . . . . . . . . . 62

A.4 A Property of normally distributed random variable . . . . . . . . . 63

A.5 Derivation of the expected value given in $(2.56) \ldots \ldots$. . . . . . . 64

$\begin{array}{ll}\text { Bibliography } & 65\end{array}$ 


\section{List of Tables}

2.1 Precoding steps $\ldots \ldots \ldots \ldots \ldots \ldots$

3.1 Initial simulation parameters . . . . . . . . . . . . . . . . . 31

3.2 Default simulation parameters for perfect CSI . . . . . . . . . . 32

3.3 Default simulation parameters for imperfect CSI . . . . . . . . . . . 39

3.4 Default simulation parameters for multicell simulation . . . . . . . . . 43

3.5 Default simulation parameters for per-user problem simulation . . . . . . 44

3.6 Default simulation parameters for antenna selection algorithms in Step 152

3.7 Default simulation parameters for antenna selection algorithms in Step $3 \quad 52$ 


\section{List of Figures}

1.1 Business Model of Wireless Network Virtualization . . . . . . . . . . . 4

1.2 An example for Limited intra-infrastructure Paradigm . . . . . . . . . . 5

1.3 An example for Cross-infrastructure Paradigm . . . . . . . . . . 6

1.4 A Framework for Wireless Network Virtualization . . . . . . . . . . . 9

2.1 Block diagram of the network . . . . . . . . . . . . . . . 15

2.2 A picture of feasible sets of the problems . . . . . . . . . . 25

3.1 A realization of a cell with 4 SPs that each has 15 users. Different colours represent users of different SPs. . . . . . . . . . . . . . . 32

3.2 Users' received SINR for different values of $\rho$ and SPs precoding . . . . 34

3.3 Norm of $\mathbf{V}^{*}$ vs $\rho$ for different number of antennas . . . . . . . . 35

3.4 Norm of $\mathbf{V}^{*}$ vs $N$ for different values of $\rho \ldots \ldots \ldots \ldots$

3.5 Histogram of channels' error . . . . . . . . . . . . . . . 38

3.6 Norm of $\mathbf{V}^{*}$ vs $\rho$ for imperfect CSI . . . . . . . . . . . . . 39

3.7 Norm of $\mathbf{V}^{*}$ vs $N$ for imperfect CSI . . . . . . . . . . . . . . 40

3.8 Maximum tolerable channel error for a given $\rho \ldots \ldots \ldots \ldots$. . . . . 41

3.9 CDF of deviation . . . . . . . . . . . . . . . . . . . . . . . . . 42

3.10 A realization of the multi-cell network under consideration. Different colours represent users of different SPs . . . . . . . . . . . . 44

3.11 Received SINR of users in the multi-cell network for different values of $K^{\prime} \quad 45$

3.12 Required transmission power vs $K^{\prime} \ldots \ldots \ldots \ldots \ldots$

3.13 Received SINR of the users for different schemes . . . . . . . . . . 47

3.14 Comparison of solutions with per-user constrain solution . . . . . . . 48

3.15 Comparison of solutions with per-user constrain solution . . . . . . . . 49

3.16 A realization of a cell with a BS with distributed antennas. The black squares represent the radio heads. . . . . . . . . . . . . 50

3.17 Comparison of different strategies of antenna selection . . . . . . . . . 51

3.18 Power saved vs different normalized $P_{\mathrm{RF}} \ldots \ldots \ldots \ldots \ldots$ 
3.19 Optimal number of antennas derived from the antennas selection algorithm 54 


\section{List of Algorithms}

1 Minimizing transmission power subject to constraints for the SPs for Perfect

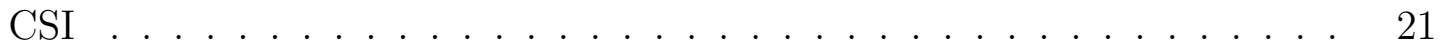

2 Minimizing transmission power subject to constraints for the SPs for im-

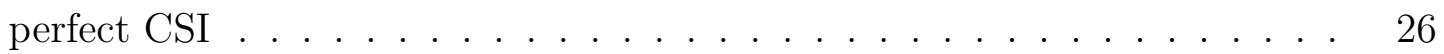

3 Finding a subset of antennas to save power $\ldots \ldots \ldots \ldots$ 


\section{Chapter 1}

\section{Introduction}

In the chapter that follows, we briefly review technologies and definitions required for developing the thesis. First, we introduce Wireless Network Virtualization, and next, we briefly review massive MIMO technology. We then present a summary of the state of the art of wireless network virtualization and highlight inadequacies of previous studies as well as our contributions. Finally, we present the organization of the thesis.

\subsection{Wireless Network Virtualization: Promises and Challenges}

The demand for higher data rate and the emergence of new technologies is increasing the capital expenses (CapEx) and operational expenses (OpEx) of service providers. This growth in expenses and the need for investment has not only demotivated the service providers to deploy modern technologies but also hindered new companies to enter the wireless industry. The concept of virtualization has been proposed to reduce these expenses of network deployment and operation by abstracting and sharing physical resources, and to facilitate the migration to newer products and technologies by decoupling distinct parts of the network.

Virtualization is to create a set of "logical" entities from a given set of "physical" entities in a manner that is transparent to users[1]. By enabling abstraction and sharing of the physical resources, it maximizes utilization of the resources while providing the required quality of service to users and enforcing the isolation and security that users need. In fact, it creates a logical representation of the entire system for users.

Due to intrinsic properties of the wireless environment such as time-varying links, attenuation, mobility and broadcast, virtualization in wireless networks has new chal- 
lenges and significant differences with wired network occur. There are different spectrum bands, many topologies, different access technologies, e.g. 3G, 4G and WiMax, with distinctive characteristics and properties in wireless networks. These properties make virtualization, specifically offering a universal virtualized framework, difficult to achieve in wireless networks.

Generally, a virtualized network is composed of Infrastructure providers (InPs) that create and manage the infrastructure (e.g. base stations, MMEs, service-gateways, access points, spectrum, etc.) and Service Providers (SPs) that utilize the virtual resources and provide services to subscribers. The InP virtualizes the resources that are owned by itself, or possibly other InPs, and splits them into slices. These slices consist of (virtualized) core networks and (virtualized) access networks corresponding to the wired slice and the wireless slice, respectively. After creating the slices, i.e. executing the virtualization of physical resources, the SPs lease these virtual resources, and operate and program them to provide end-to-end services to end-users, without knowing the underlying physical architecture of the InP. Virtualization by the InP makes physical resources behind the slices hidden to the SPs and creates a logical representation of the entire system.

Wireless network virtualization can have a very broad scope ranging from spectrum sharing, infrastructure virtualization, to air interface virtualization. Our main focus in this thesis will be on the air interface virtualization. However in the following sections we will review all paradigms and aspects of wireless network virtualization in order to provide a better understanding which will help in developing the next chapters.

\section{Motivations for Virtualizing Wireless Networks}

Successes of wired network virtualization and its benefits motivated virtualizing wireless networks. By enabling abstraction and sharing of the resources in wireless networks among different entities, network virtualization similarly reduces the overall expenses of network deployment and operation. These expenses include the capital expenses and operation expenses of radio access networks (RANs), as well as core networks (CNs). Also by decoupling different parts of a network, wireless network virtualization helps facilitate emergence and migration of new technologies. Furthermore, it provides a substrate for evaluating new ideas and research coming from the academia in a real wireless network with affordable expenses.

Moreover, SPs, who may provide some specific services (e.g., VoIP, video call, overthe-top services), become decoupled from InPs. This makes them help each other in the manner that SPs focus on attracting more users, while InPs produce more revenue by leasing the isolated virtualized networks to them and offering new services [2]. Further, 
wireless network virtualization creates a competition environment, which results in better overall system performance and spectrum utilization which from that all the entities benefit. In such systems, users will simply subscribe to services or applications they desire and the SPs only deliver the access service.

\section{Business Model of WNV}

Generally, in network virtualization, irrespective of whether it is in wired or wireless environments, some entities own and operate physical resources, including the licensed spectrum, radio access networks (RANs), backhaul, transmission networks (TNs), and core networks $(\mathrm{CNs})$, and some parties utilize the virtual resources. So, it is logically possible to view the network as being composed of Infrastructure providers or InPs that create and manage the infrastructure (e.g., base stations, MMEs, S-Gateways, APs, spectrum, etc.) and Service Providers or SPs, which actually provide various services to subscribers [1]. The InPs thus virtualized the resources that are own by itself or some other InP's and split them into slices. The physical resources behind these slices are transparent to SPs and a slice creates an illusion that it is an entire system by itself. This slice consists of its own (virtualized) core network and (virtualized) access network corresponding to the wired slice and the wireless slice, respectively. After creating the slices, i.e. executing the virtualization of physical resources, SPs lease these virtual resources, operate and program them to provide end-to-end services to end-users, without actually knowing the underlying physical architecture of the InPs.

The terminology used in wireless network virtualization is not yet unified. For instance, in [2], the authors refer to mobile network operators (MNO) as entities that execute the virtualization while some papers refer InPs as entities which are only responsible for owing and leasing wireless network resources. In [3], the authors suggested to further decouple the roles into more specialized roles, including InP, mobile virtual network provider (MVNP), mobile virtual network operator (MVNO) and SP [4],[5],[6] and suggested the following functions as described below.

1. InP: It owns the infrastructure and wireless network resources. However, the spectrum resources may or may not be owned by $\mathrm{InP}$.

2. MVNP: It leases the network resources and execute the virtualization. They may also have some licensed spectrum such that they do not need to request spectrum resources from InP. In some papers, MVNP is called mobile virtual network enabler $(\mathrm{MVNE})$. 


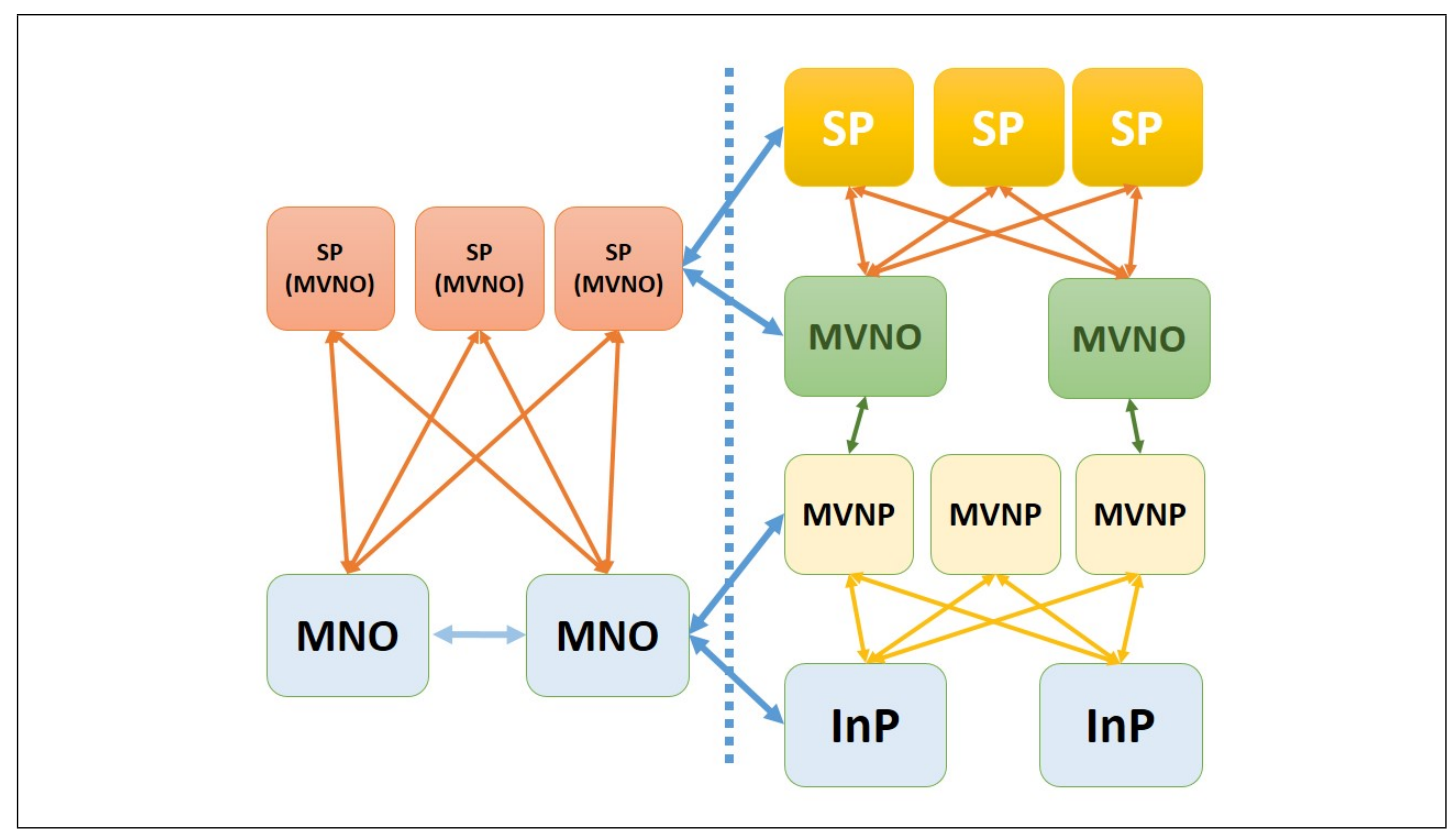

Figure 1.1: Business Model of Wireless Network Virtualization

3. MVNO: It operates and assigns the virtual resources to SPs. Meanwhile, in some approaches, MVNOs consists of the roles of both MVNOs and MVNPs.

4. SP: It leases resources from MVNO and concentrates on providing services to its subscribers based on the virtual resources.

This four level model provides more opportunities in the market and simplifies the design by decoupling the roles of parties in wireless network virtualization. However, more coordination and interface mechanism is required which increases system's complexity [3].

In the rest of the thesis and particularly in Chapter 2, we refer to InP as a party whose role consists of InP and MVNP's roles as expressed in the above four-level model and refer to SP as a party whose role consists of MVNO and SP mentioned above.

\section{Requirement of Wireless Network Virtualization}

Wireless network virtualization, to a certain extent, can be considered as natural extension of wired network virtualization and virtualization of end systems such as servers and cloud computing systems, which is currently widespread. Similar to them, it necessitates the implementation of the some basic requirements. These requirements are listed as follows [3].

- Coexistence: Wireless network virtualization should provide the creation of several virtual resources to coexist on a common physical infrastructure. As such, it is 


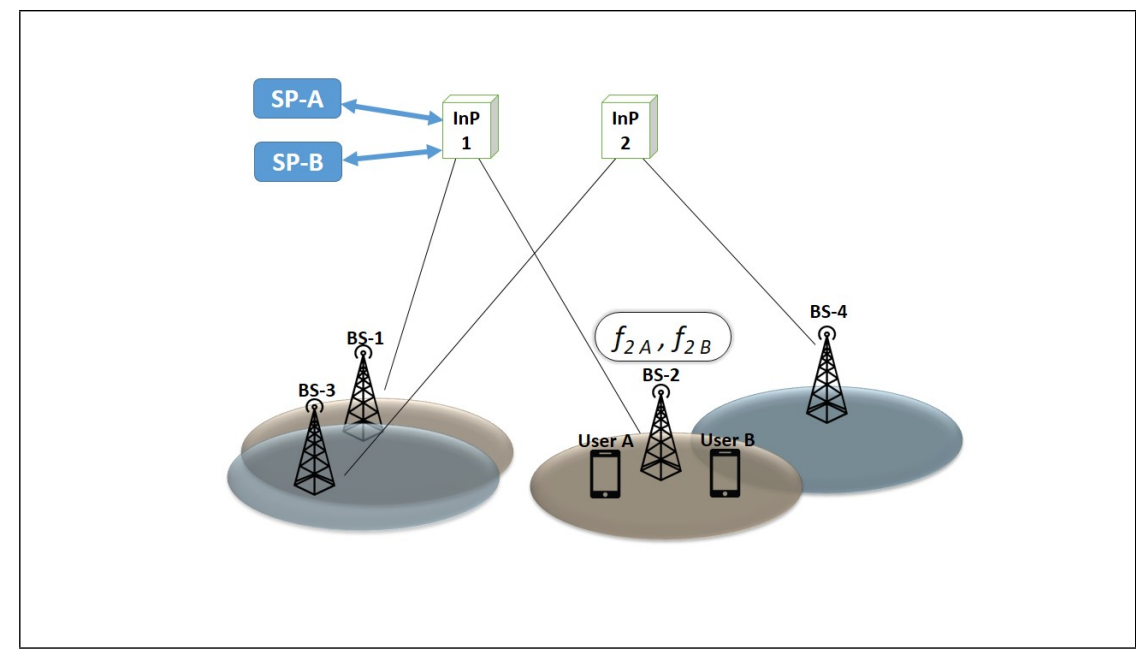

Figure 1.2: An example for Limited intra-infrastructure Paradigm

the purpose of virtualization to allow multiple systems to run on the same physical resources.

- Flexibility, manageability and programmability: wireless network virtualization should provide different services with independent networking through decoupling the components of the network. Virtualization will also allow SPs to customize their services and provide an opportunity to use new products in the network.

- Isolation: Isolation ensures that any changes in others slices, system configuration or network topology do not impact the slice or the service offered to a given SP. Isolation is the primary requirement that enable the perception that a slice is the entire network.

- Efficiency: Wireless network virtualization enables a network to be utilized better in terms of physical resources and spectrum.

\section{Paradigms for Wireless Network Virtualization}

In [1], the author proposed three paradigms for wireless network virtualization. We briefly describe these paradigms here for better understanding of wireless network virtualization and as an approach toward virtualizing wireless networks.

- Limited Intra-infrastructure: In this paradigm, virtualization is only considered within each InP. Here, InP is assumed to be capable of sharing its resources and spectrum among different SPs and across different radio access technologies (RAT). A simplified example of this paradigm is depicted in Fig 1.2. In this example, two 


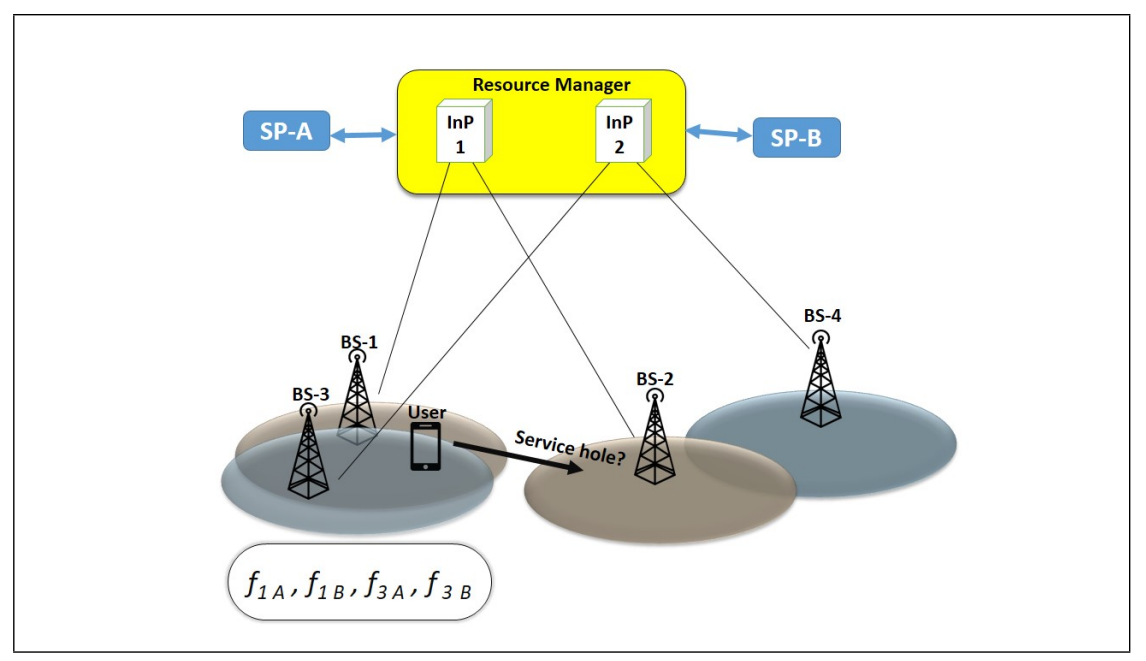

Figure 1.3: An example for Cross-infrastructure Paradigm

SPs A and B lease a certain amount of resources from InP 1 at each cell. Obviously, the multiplexing gain of this paradigm is likely to be low but it can be viewed as the first step toward virtualization. (This is the paradigm that we adopted to consider massive MIMO in wireless network virtualization, but this approach does not limit our framework, which can be extended to the other paradigms).

- Cross-infrastructure: In this paradigm they assumed that the resources of InPs can be possibly aggregated across InPs to be shared among SPs. A simplified example of this paradigm is depicted in Fig 1.3. In this example BS1 and BS2 belong to InP 1 while BS3 and BS4 belong to InP2. A central unit is required, which can be considered as the role MVNP, to ensure cooperation and isolation among SPs.

- Universal Virtualization: In this paradigm, the virtualization is pervasive and the wireless network virtualization does not need to make any assumptions about SPs and InPs. SPs select the services they want and program it based on their requirements and preferences. It can be on demand and be specified in whatsoever details that SPs desire. This cloud like virtualization is the ultimate goal of virtualization and due to its complicated management and economic issues many challenges yet to be studied in future work.

\section{Challenges of Wireless Network Virtualization}

There are several challenges that wireless network virtualization encounters regardless of the paradigm adopted or the scope of virtualization. Some of these challenges are summarized here and mostly inspired from [1] and [3]. 
1. Interfacing: A common and well-defined interface is required for controlling and managing the coordinating of the network.

2. Resource Allocation: It is one of the primary challenge of wireless network virtualization which directly effect efficient, ensure isolation and maintain quality of services of SPs.

3. Resource Discovery: This is essential particularly when cross infrastructure or universal infrastructure paradigms is adopted. In order to utilize all resources efficiently, MVNP or InP should be aware of all available resources.

4. Isolation: Isolation is that any changes in the configuration of the network should not impact the service associated to a slice.

5. Mobility Management: Coordinating and managing mobility in wireless network virtualization can be easier than traditional network. Wireless network virtualization facilitates this mobility management through spectrum/infrastructure sharing and protocols exist between SPs and InPs.

6. Security: Users privacy and avoiding leaking users' information from an InP to an SP should by accurately addressed.

7. Governance regulations: The problems including the ownership of the spectrum, physical infrastructure should be argued and justified economically. For instance, it is unlikely that legacy operator will be willing to easily share their resources unless strong economic and regulatory reasons enforce it.

\section{Wireless Network Virtualization Framework}

According to [3] and based on the studies conducted by the authors, wireless network virtualization can be composed of four main components: radio spectrum resource, wireless network infrastructure, wireless virtual resource, and wireless virtualization controller. In the next paragraphs we briefly describe each component.

1. Radio spectrum resource: Radio spectrum resource is one of the most important resources in wireless communications which usually gives an operator competency. Generally, Radio spectrum resource refers to the licensed spectrum or some dedicated free spectrum. Sharing all or part of the licensed spectra owned by operators and allocating it to other SPs is one of the main challenge of wireless network virtualization, either from technical aspects or from regulatory aspects. 
2. Wireless network infrastructure: It refers to the whole wireless physical substrate network, including sites (towers and antennas), base stations (macrocell,smallcell, relay, $\mathrm{RF}$, baseband processors, radio resource controllers, etc. in cellular networks), access points (in wireless local area networks (WLANs)), core network elements (gateway, switch, routers, etc.), transmission networks (backhaul and links between RANs and $\mathrm{CN}$ ). These infrastructure are the foundation of wireless network and occupy the majority of the investment of the operators.

3. Wireless virtual resources: These are the resources created by slicing wireless network infrastructure and spectrum into multiple virtual slices. The authors in [3] suggested three levels of virtualization which we present here.

(a) Spectrum-level slicing: Spectrum-level slicing in fact extends dynamic spectrum access and spectrum sharing [3]. Spectra can be sliced in time, frequency, space or code.

(b) Infrastructure-level slicing: In infrastructure-level slicing, the physical network elements, e.g., antennas, BSs, processor hardware and routers, are virtualized to support sharing by multiple operators. It is the case when an MVNO owns a part of network or spectra, or aims to extend its coverage, and thus lease infrastructure and hardware from an InP in a certain area. Therefore, the InP has to virtualize this physical resources into slices of virtual infrastructure and virtual machines.

(c) Network-level slicing: Network-level slicing is the ideal ultimate goal and is similar to the universal paradigm mentioned before.

4. Wireless virtualization controller: It is used for realizing customability, manageability and programmability of virtual slices available to SPs. Through wireless virtualization controller, the control plane is decoupled from data plane and SPS can customize the virtual resource within their own virtual slices.

\subsection{Massive MIMO}

The technology that this thesis aimed to virtualize is massive MIMO. Massive MIMO wireless communications refers to equipping cellular base stations (BSs) with a large number of antennas, typically on the order of 100 or more. The number of degrees of freedom that this provides allows effective concentration of power as well as interference suppression over multiple mobile devices. 


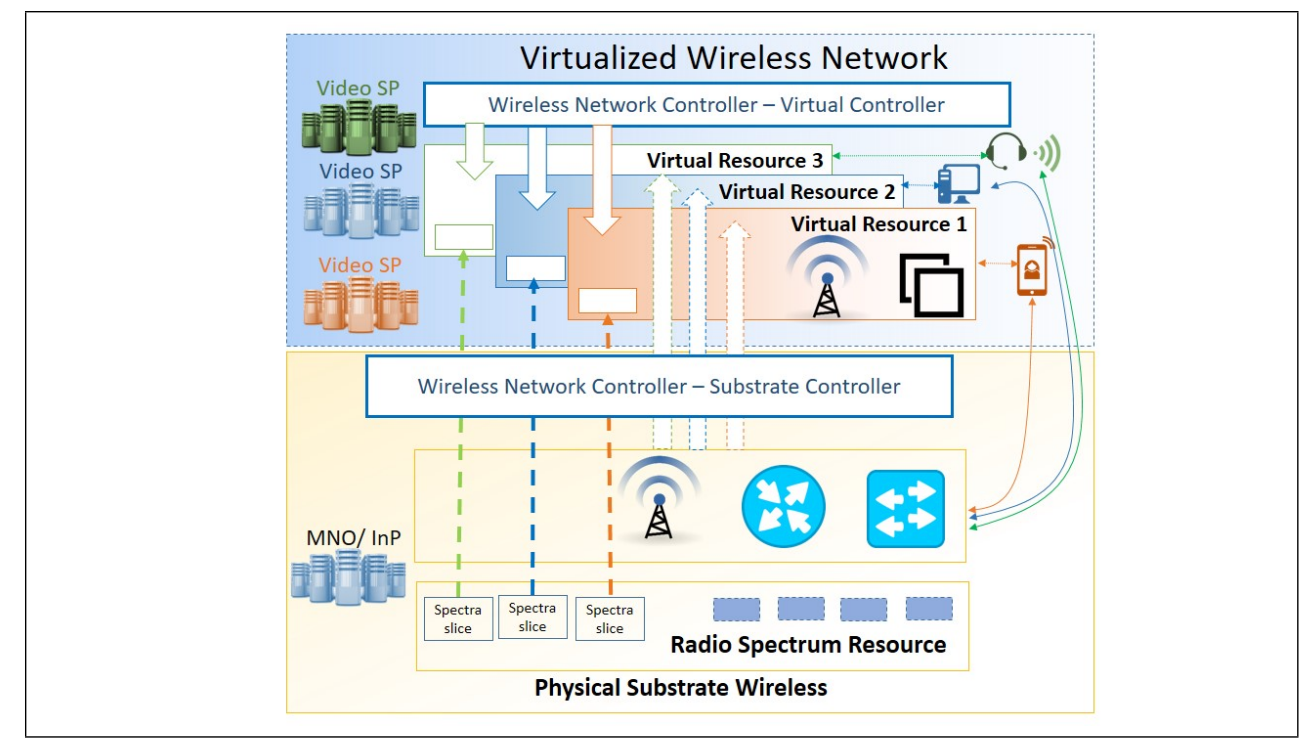

Figure 1.4: A Framework for Wireless Network Virtualization

Massive MIMO first proposed in [7] and the authors argued that as the number of antennas grows larger, the effects of uncorrelated noise and small-scale fading are eliminated and the required transmitted energy per bit vanishes. Further, They showed that close to optimal performance can be achieved by using simple linear signal processing approaches, such as matched filter(MF) precoding/detection. In [8], the authors showed that each single-antenna user in a massive MIMO system can scale down its transmit power proportional to the number of antennas at the BS with perfect channel state information (CSI) or to the square root of the number of BS antennas with imperfect CSI, to get the same performance as a corresponding single-input single-output (SISO) system.

While massive MIMO bring about many advantageous to wireless networks, many challenges remain to be addressed. For instance, since the maximum number of orthogonal pilot sequences that can exist is upper-bounded by the duration of the coherence interval divided by the channel delay spread, pilots should be re-used across the cells. This causes negative consequences which is referred to as pilot contamination. Downlink beamforming based on the contaminated channel estimate results in interference directed at those terminals that share the same pilot sequence. It is argued for instance in [7] that pilot contamination constitutes an ultimate limit on performance when the number of antennas is increased without bound, at least with receivers that rely on pilot based channel estimation. Other challenges including low cost hardware, channel reciprocity assumption, etc. have been mentioned in the literature as well. 


\section{Precoding in Massive MIMO}

As we later investigate the problem of precoding in wireless network virtualization and massive MIMO, we briefly review the basic linear precoding techniques used in wireless networks.

Two types of precoding have been studied in the MIMO literature: linear and nonlinear precoding. Compared with linear precoding methods, non-linear methods, such as dirty-paper-coding (DPC) [9], vector perturbation(VP) [10], and lattice-aided methods [11] have better performance albeit with higher implementation complexity. However, with an increase in the number of antennas at the BS, linear precoders, such as MF(Match Filter) and ZF(Zero-Forcing), are shown to be near-optimal [12].

When MF is used, the transmit signal from the BS can be expressed as

$$
\mathbf{x}^{\mathrm{MF}}=\frac{1}{\sqrt{\alpha}} \mathbf{H}^{H} \mathbf{s}
$$

where $\alpha$ is the normalization factor. When ZF is used, the transmit signal from the BS can be expressed as

$$
\mathbf{x}^{\mathrm{MF}}=\frac{1}{\sqrt{\alpha}} \mathbf{H}^{*}\left(\mathbf{H}^{T} \mathbf{H}^{*}\right)^{-1} \mathbf{s}
$$

For Regular Zero Forcing, a regularized term is added prior to inversion and the transmit signal at the BS is expressed as

$$
\mathbf{x}^{\mathrm{MF}}=\frac{1}{\sqrt{\alpha}} \mathbf{H}^{*}\left(\mathbf{H}^{T} \mathbf{H}^{*}+\delta \mathbf{I}\right)^{-1} \mathbf{s}
$$

The performance of these precoding schemes has been well studied, particularly when the number of antennas at the BS is much larger than the number of users and tends to infinity. The ZF precoder outperforms MF in the region of high spectral efficiency while $\mathrm{MF}$ is better in the region of low spectral efficiency.

\subsection{Literature Review}

Wireless network virtualization has been studied under two categories in the literature. The first category focuses on resource allocation and spectrum partitioning and enforcing fairness among users, while the second category studies how virtualization can be applied to the technologies in wireless networks. This work belongs to the second category and studies the virtualization of massive MIMO technology. 
In the first category, work focuses on spectrum and resource management, business model of wireless network virtualization and market profit views of it [13], [14], [15]. In such cases, two types of interactions are studied between users and SPs or between SPs and InPs. Such interactions usually are modelled as stochastic games and the existence of the Nash equilibrium can result in an optimum price for spectrum.

In [13], the authors modelled the bandwidth allocation problem as a non-cooperative game model and proposed an iterative algorithm that is based on finding the Nash equilibrium. However, since bandwidth is directly used to represent the radio resource in [13], the relationship between capacity and radio resource is not considered.

In [16],the authors modelled the interaction among the SPs and the InP, which they called network operator, as a stochastic game. The SPs play on behalf of their users and the game is regulated under network operator through the Vickrey-Clarke-Groves mechanism. In order to deal with the strong coupling between the SPs decisions and to avoid congestion, they introduced conjectural prices. The strategy for the SPs is to select price and announce their preferred rates. They proved that there is a Nash equilibrium and the SPs have to truthfully announce their value functions. Despite the interesting results of the work and the depth of technical parts, they do not guarantee the isolation among the SPs and do not consider other requirements of WNV such as customization.

In [17], which is one of the primary work in wireless network virtualization and is more a descriptive paper, the authors modelled the interaction between the spectrum owner and the SPs as an auction in a way that each SP bids for a part of band and a duration and the spectrum with price for the bid. The spectrum owner maximizes the profit taking into account the availability of the spectrum and allocation of paired and unpaired bands.

In [15], the authors focused on wireless network virtualization as a testbed in a manner that there are some experiments waiting to be conducted, and the objective is to schedule these experiments by taking advantage of time and space. Their goal is to maximize resource utilization in the premise of ensuring link quality.

In [14], the authors proposed an online embedding algorithm, assigning wireless resources to SPs, which includes online scheduling. They modelled the problem in a way that the SPs request a rectangular area in time-frequency grid, substrate resource, and specify the lifetime of their requests. The InP aims to maximize the occupied units in the substrate resource. The main weakness of this work is that they failed to justify why they model the requests as a rectangular area. In addition, they ignore the question how the requirements of a virtualized network such as isolation are addressed in their model.

In the second category, research focuses on the implementation of wireless virtualiza- 
tion on a particular platform e.g.LTE, Wi-Fi or WiMax ( [18],[19], [20],[21],[22], and [23]). Such studies consider case studies and use simulations to evaluate the technical benefits of virtualization. Compared to the work that looks at spectrum pools, the related work on the technical implementation is limited.

In [19],[18] and [20], the author considered a Hypervisor on top of the physical layer in the eNodeB in order to virtualize the base station. This entity is responsible for scheduling the physical resources and air interface resources among different virtual instances. The Hypervisor collects information from virtual eNodeBs to schedule them by splitting the spectrum. This scheduling can be based on different criteria such as bandwidth, data rate, power, predefined contract, etc. In this work they concentrated on a contract based splitting by maintaining the average bandwidth allocated to each SP. The approach presented in this work is a practical mechanism to realize virtualization

The virtualization of WLAN access points has been considered in [21],[22],[23]. For instance in [22], the authors proposed an architecture called "SplitAp" to provide fairness in resource partitioning for group of WLAN users.

Although these studies show the importance and potential of wireless network virtualization, there is little published on the relation between massive MIMO and wireless network virtualization. The authors of [24] studied resource provisioning in wireless networks via massive MIMO. However, they failed to fully explain how their model ensures the requirements of virtualization. For instance, it is unspecified how the SPs can program their services, e.g. design precoding matrix or perform scheduling. The other weakness is with some of the assumptions they made in these studies. They assumed that different SPs use a disjoint set of antennas without fully explaining the reason. They also assumed the resource blocks are orthogonal. These weaknesses are addressed in this thesis by proposing a framework for the network and introducing a novel precoding algorithm.

Although the precoding problem has been well studied in wireless communication, and different schemes of precoding have been proposed and studied such as in [25],[10], new challenges arise when it comes to wireless network virtualization. Since each SP does not have access to the channel information of the users of other SPs, handling the interference between the users of different SPs can potentially be challenging. For instance, if the SPs use typical schemes of precoding and have the InP use their precoding matrices without considering the other SPs, the system will likely incur a large amount of interference. We therefore propose that the InP should manage this interference, and it should be in a way that the users of the SP receive nearly the same signal that the SPs have designed for them. This problem has not been treated in traditional precoding 
schemed in a wireless network.

In this thesis we study this problem in detail and consider the challenges arise when wireless network virtualization applies on massive MIMO base station. We first propose a framework that addresses the requirements of wireless network virtualization. We then mathematically formulate the problems associated to this framework and propose a solution for each. We summarize our main contributions below:

- We formally formulate the precoding problem in wireless network virtualization when perfect CSI is available. We propose a simple iterative algorithm to find the optimal precoding with respect to minimum transmission power.

- We extend this precoding problem to non-perfect CSI and formulate it with chance constraints. Despite the intractability of problems with chance constraints, we propose a simple and effective solution, and show that the solution promises very close to optimal performance by providing a lower bound for the optimal solution.

- We show our formulation can address other concerns such as inter-cell interference cancellation.

- We develop methods to select transmission antennas, and by numerical simulations we show the effectiveness of the proposed antennas selection algorithms.

\subsection{Thesis Outline}

The rest of the thesis is composed of three chapters. It continues with Chapter 2 in which we state the challenges of employing massive MIMO in wireless network virtualization. We define our framework and mathematically formulate the precoding problem. We consider both perfect channel information and imperfect channel. Simulation performance and discussion are presented in Chapter 3 in which we evaluate the performance of the solutions we proposed and also investigate the solutions for different set of parameters. Finally, Chapter 4 concludes the thesis and summarizes the impacts and contributions of this work and suggests potential directions to extend this work. 


\section{Chapter 2}

\section{System Model and Problem Statement}

\subsection{System Model}

For convenience and without loss of generality we assume that all the components in the virtualized wireless network are encompassed into two entities: Service Providers (SPs) and one Infrastructure Provider (InP). The SPs are responsible for serving the subscribers and programming their services, and the InP owns the infrastructure, execute virtualization and manage the services. We also assume that other parts of the network including the core network and computational resources are already virtualized and can be utilized by the SPs and the InPs.

We initially consider a one-cell cellular network but later generalize it to multi-cell networks. This one-cell network is comprised of an InP that owns a base station (BS) with $N$ antennas, and $M$ SPs with their own schedulers and precoders. The SPs are able to design the desirable precoding matrix for its own users. Denote $K_{m}$ as the number of users of SP $m, \mathbf{H}_{m} \in \mathbb{C}^{K_{m} \times N}$ as the flat fading channel matrix of the users of SP $m$ from the base station, and $\mathbf{W}_{m}$ as the precoding matrix designed by SP $m$ (Figure 2.1). The InP is responsible for virtualizing the base station that is assumed to be executed in a manner transparent to the users. For our purpose, it means that the users of a SP should receive signals with a defined throughout and quality of service by the SP through the precoding matrix. It is worth noting that the InP cannot simply transmit using a simple summation of the precoding matrices designed by the SPs since that would lead severe inter-SPs interference. The InP indeed should design a new precoding matrix, V, such that the users of each SP receive the desired signals designed for them (by each SP 


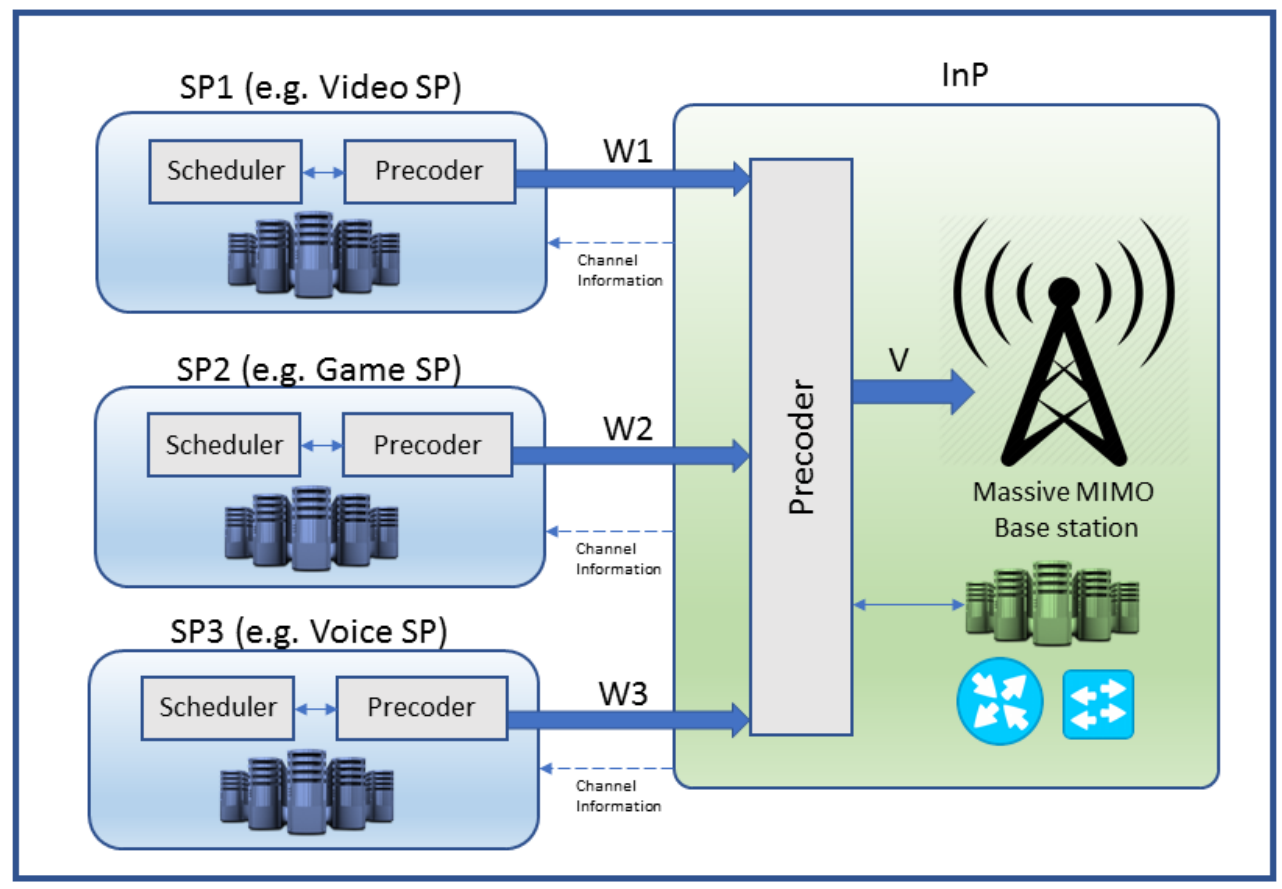

Figure 2.1: Block diagram of the network

through $\mathbf{W}_{m}$ ) with low level of interference from the signal intended for the other SPs.

In addition to executing virtualization, and satisfying constraints that come from the requirement of it, the $\mathrm{InP}$ is concerned with additional internal constraints such as total transmit power. This work take power minimization as the objective of the InP and formulate the problem with this objective.

For the sake of generality, we assume that the InP provides the channel information of a subset of antennas to each SP, e.g. channel information of $N_{m}$ of antennas to SP $m$. This may be preferable for the SPs and the InP as the overhead of channel information exchange would be less. We will try to justify it further in Chapter 3. Assume $N_{m}$ (besides other physical parameters) are predetermined based on a long term agreement between the SPs and the InP.

According to the contract and the requirements of wireless network virtualization, three necessary steps should be performed by the InP and the SPs. These steps are expressed as follows.

First, for each SP $m$, the InP selects $N_{m}$ antennas from all the available antennas and communicate the corresponding channel information $\mathbf{G}_{m}$ to the SP $m$.

Second, the SPs design the precoding matrices $\mathbf{W}_{m}$ for their users and communicate 


\begin{tabular}{|c|l|l|}
\hline Step & InP & SP \\
\hline 1 & $\begin{array}{l}\text { Chooses subsets of antennas }\left(N_{m} \text { an- }\right. \\
\text { tennas for the SPs from the available } \\
\text { set of antennas and provide the SPs } \\
\text { with the corresponding channel infor- } \\
\text { mation. }\end{array}$ & \\
\hline 2 & $\begin{array}{l}\text { Design the final precoding matrix using } \\
\text { the indicated algorithms: }\end{array}$ & $\begin{array}{l}\text { Design precoding matrix by using the } \\
\text { channel information and the current } \\
\text { state of the system. }\end{array}$ \\
\hline $\begin{array}{l}\text { In Section } 2.2 \text { if the CSI is per- } \\
\text { fectly known } \\
\text { In Section } 2.3 \text { if the CSI is not } \\
\text { perfectly known }\end{array}$ & \\
\hline
\end{tabular}

Table 2.1: Precoding steps

them to the InP.

Third, the InP collects all $\mathbf{W}_{m}$ and designs precoding matrix $\mathbf{V}$.

For better reference in future, we also mention these steps in Table 2.1.

We assume that the InP has an algorithm to choose a subset of antennas for each SP. For the sake of completeness, we will suggest such an algorithm in Section 2.5.1. We also assume that the SPs have their own scheduling and precoding algorithms to design their favourable precoding matrix. These algorithms can be viewed as software running at the base station. In Step 3, the InP can use a subset of antennas for the transmission and switch of the remaining antennas. In this case, the InP jointly finds a subset of antennas for transmission and design the final precoding matrix. We propose a greedy algorithm given in Section 2.5.2 which finds the optimal subset of antennas.

Finally and as a reference, We list the notations introduced in this section and will be used in the rest of this work.

- Matrices are set in bold-face upper-case letters whereas vectors are set in bold-face lower-case letters.

- In vectors and matrix, comma is used for horizontal concatenation and semicolon for vertical concatenation. 
- The superscript ${ }^{H}$ stands for Hermitian transpose, ${ }^{T}$ stands for transpose and.$^{+}$ stands for pseudo inverse.

- $\mathbf{I}$ is the identity matrix.

- $\|$.$\| denote Frobenius norm.$

- $\otimes$ is used for Kronecker product.

\subsection{Power Minimization with Perfect CSI}

\subsubsection{Problem Statement}

Suppose that the InP has communicated to each SP their channel information, $\mathbf{G}_{m}$, for the transmission period under consideration. The transmission period can be permanent, semi-permanent, or dynamic on a frame or subframe basis. Let $\mathbf{x}_{m} \in \mathbb{C}^{K_{m}}$ represent the symbols (messages) for the users of SP $m$ and define $\mathbf{x}$ as

$$
\mathbf{x}=\left[\mathbf{x}_{1} ; \ldots ; \mathbf{x}_{M}\right] .
$$

Without loss of generality, assume that the messages of the SPs are zero-mean, uncorrelated, and normalized to 1, i.e.,

$$
\begin{gathered}
\mathbb{E} \mathbf{x}_{m}=0 \\
\mathbb{E} \mathbf{x}_{m}^{H} \mathbf{x}_{n}= \begin{cases}0 & \text { if } m \neq n \\
1 & \text { if } m=n .\end{cases}
\end{gathered}
$$

Let $\mathbf{V}=\left[\mathbf{V}_{1}, \ldots, \mathbf{V}_{M}\right]$ be the precoding designed by the InP. Therefore the users of $\mathrm{SP} m$ receive

$$
\mathbf{y}_{m}=\mathbf{H}_{m} \mathbf{V}_{m} \mathbf{x}_{m}+\sum_{i \neq m} \mathbf{H}_{m} \mathbf{V}_{i} \mathbf{x}_{i}
$$

The precoding matrix $\mathbf{V}$ should be designed in a way that the received signal in (2.3) does not deviate significantly from the signal that SP $m$ expects that its users will receive, i.e.

$$
\mathbf{y}_{m}^{\prime}=\mathbf{G}_{m} \mathbf{W}_{m} \mathbf{x}_{m}
$$


Mathematically, for each SP $m$, the InP should satisfy the following inequality

$$
\mathbb{E}_{\mathbf{x}}\left\|\mathbf{y}_{m}-\mathbf{y}_{m}^{\prime}\right\|^{2} \leq \mathcal{I}_{m}^{2}
$$

where $\mathcal{I}_{m}$ is a predefined threshold, defined in the agreement between the SP and the InP. By substituting (2.3) and (2.4), this can be re-written as

$$
\mathbb{E}_{\mathbf{x}}\left\|\mathbf{H}_{m} \mathbf{V} \mathbf{x}-\mathbf{G}_{m} \mathbf{W}_{m} \mathbf{x}_{m}\right\|^{2} \leq \mathcal{I}_{m}^{2} \quad \forall m
$$

and by taking the expected value, the left-hand side is given by

$$
\begin{aligned}
& \mathbb{E}_{\mathbf{x}}\left\|\mathbf{H}_{m} \mathbf{V} \mathbf{x}-\mathbf{G}_{m} \mathbf{W}_{m} \mathbf{x}_{m}\right\|^{2} \\
= & \mathbb{E}_{\mathbf{x}}\left\|\mathbf{H}_{m} \sum_{i} \mathbf{V}_{i} \mathbf{x}_{i}-\mathbf{G}_{m} \mathbf{W}_{m} \mathbf{x}_{m}\right\|^{2} \\
= & \mathbb{E}_{\mathbf{x}} \operatorname{Trace}\left(\mathbf{H}_{m} \sum_{i} \mathbf{V}_{i} \mathbf{x}_{i}-\mathbf{G}_{m} \mathbf{W}_{m} \mathbf{x}_{m}\right)\left(\mathbf{H}_{m} \sum_{i} \mathbf{V}_{i} \mathbf{x}_{i}-\mathbf{G}_{m} \mathbf{W}_{m} \mathbf{x}_{m}\right)^{H} \\
= & \operatorname{Trace}\left(\mathbf{H}_{m} \mathbf{V}_{m}-\mathbf{G}_{m} \mathbf{W}_{m}\right)\left(\mathbf{H}_{m} \mathbf{V}_{m}-\mathbf{G}_{m} \mathbf{W}_{m}\right)^{H}+\sum_{i \neq m} \operatorname{Trace}\left(\mathbf{H}_{m} \mathbf{V}_{i}\right)\left(\mathbf{H}_{m} \mathbf{V}_{i}\right)^{H} \\
= & \left\|\mathbf{H}_{m} \mathbf{V}_{m}-\mathbf{G}_{m} \mathbf{W}_{m}\right\|_{F}^{2}+\sum_{i \neq m}\left\|\mathbf{H}_{m} \mathbf{V}_{i}\right\|_{F}^{2}
\end{aligned}
$$

where $\|\cdot\|_{F}$ denotes the Frobenius norm, i.e.

$$
\|\mathbf{X}\|_{F}^{2}=\operatorname{Trace}\left(\mathbf{X X}^{H}\right) .
$$

We focus on power minimization as the objective of the InP subject to the SPs performance expectations given in (2.6). The InP thus solves the optimization problem given by

$$
\begin{aligned}
\min _{\mathbf{V}} & \|\mathbf{V}\|^{2} \\
\text { subject to: } & \left\|\mathbf{H}_{m} \mathbf{V}_{m}-\mathbf{G}_{m} \mathbf{W}_{m}\right\|^{2}+\sum_{i \neq m}\left\|\mathbf{H}_{m} \mathbf{V}_{i}\right\|^{2} \leq \mathcal{I}_{m}^{2} \quad \forall m .
\end{aligned}
$$

The following lemma provides a sufficient condition for the feasibility of the problem and also describes a property of the optimal solution.

Lemma 2.2.1. If $K=\sum_{i} K_{i} \leq N$, the problem (2.13-2.14) is always feasible and at the optimal solution all the constraints are active.

We provided the proof in Appendix A.1. 


\subsubsection{Proposed Solution for Perfect CSI}

When perfect channel information is available, the optimization problem given in (2.132.14 ) is a convex program. One can use commercial solvers to find the optimal solution to this problem, or follow the iterative algorithm derived below.

The dual Lagrangian function of the problem given in (2.13-2.14) is defined by

$$
\begin{aligned}
L(\mathbf{V}, \boldsymbol{\lambda})= & \|\mathbf{V}\|^{2}+\sum_{m} \lambda_{m}\left(\left\|\mathbf{H}_{m} \mathbf{V}_{m}-\mathbf{G}_{m} \mathbf{W}_{m}\right\|^{2}+\sum_{i \neq m}\left\|\mathbf{H}_{m} \mathbf{V}_{i}\right\|^{2}-\mathcal{I}_{m}\right) \\
= & \sum_{m} \operatorname{Trace}\left\{\mathbf{V}_{m} \mathbf{V}_{m}^{H}\right\}+\sum_{m} \lambda_{m}\left[\operatorname{Trace}\left\{\mathbf{H}_{m}\left(\sum_{i} \mathbf{V}_{i} \mathbf{V}_{i}^{H}\right) \mathbf{H}_{m}^{H}\right)\right\}-\operatorname{Trace}\left\{\mathbf{H}_{m} \mathbf{V}_{m} \mathbf{W}_{m}^{H} \mathbf{G}_{m}^{H}\right\} \\
& \left.-\operatorname{Trace}\left\{\mathbf{G}_{m} \mathbf{W}_{m} \mathbf{V}_{m}^{H} \mathbf{H}_{m}^{H}\right\}+\operatorname{Trace}\left\{\mathbf{G}_{m} \mathbf{W}_{m} \mathbf{W}_{m}^{H} \mathbf{G}_{m}^{H}\right\}-\mathcal{I}_{m}\right]
\end{aligned}
$$

and the dual function is

$$
g(\boldsymbol{\lambda})=\min _{\mathbf{V}} L(\mathbf{V}, \boldsymbol{\lambda})
$$

By taking derivative with respect to $\mathbf{V}_{m}^{*}$ and setting it to zero we have

$$
\frac{\partial L}{\partial \mathbf{V}_{m}^{*}}=\mathbf{V}_{m}+\sum_{i} \lambda_{i} \mathbf{H}_{i}^{H} \mathbf{H}_{i} \mathbf{V}_{m}-\mathbf{H}_{m}^{H} \mathbf{G}_{m} \mathbf{W}_{m}=0
$$

Therefore,

$$
\begin{aligned}
& \mathbf{V}_{m}^{*}=\left(\mathbf{I}+\lambda_{i} \mathbf{H}_{i}^{H} \mathbf{H}_{i}\right)^{-1} \mathbf{H}_{m}^{H} \mathbf{G}_{m} \mathbf{W}_{m} \\
& \mathbf{V}^{*}(\boldsymbol{\lambda})=\left[\mathbf{V}_{1}^{*}, \ldots, \mathbf{V}_{M}^{*}\right]
\end{aligned}
$$

and

$$
g(\boldsymbol{\lambda})=L\left(\boldsymbol{\lambda}, \mathbf{V}^{*}(\boldsymbol{\lambda})\right)
$$

Since strong duality holds, we simply need to find the solution to the optimization problem defined by

$$
\max _{\boldsymbol{\lambda} \geq 0} g(\boldsymbol{\lambda})
$$

Lemma 2.2.2. For function $g(\boldsymbol{\lambda})$ defined in (2.21), the following choice of $\mathbf{d}$ is a sub- 
gradient for $g(\boldsymbol{\lambda})$ :

$$
\begin{aligned}
\mathbf{d} & =\left(d_{1}, \ldots, d_{M}\right)^{T} \\
d_{i} & =\left(\left\|\mathbf{H}_{m} \mathbf{V}_{m}^{*}-\mathbf{G}_{m} \mathbf{W}_{m}\right\|^{2}+\sum_{i \neq m}\left\|\mathbf{H}_{m} \mathbf{V}_{i}^{*}\right\|^{2}\right),
\end{aligned}
$$

where $\mathbf{V}^{*}$ is the optimizing variable in the minimization problem in the definition of $g(\boldsymbol{\lambda})$.

Proof. We have

$$
\begin{gathered}
g\left(\boldsymbol{\lambda}^{\prime}\right)=\min _{\mathbf{V}}\|\mathbf{V}\|^{2}+\sum_{m} \lambda_{m}^{\prime}\left(\left\|\mathbf{H}_{m} \mathbf{V}_{m}-\mathbf{G}_{m} \mathbf{W}_{m}\right\|^{2}+\sum_{i \neq m}\left\|\mathbf{H}_{m} \mathbf{V}_{i}\right\|^{2}-\mathcal{I}_{m}\right) \\
\leq\left\|\mathbf{V}^{*}\right\|^{2}+\sum_{m} \lambda_{m}^{\prime}\left(\left\|\mathbf{H}_{m} \mathbf{V}_{m}^{*}-\mathbf{G}_{m} \mathbf{W}_{m}\right\|^{2}+\sum_{i \neq m}\left\|\mathbf{H}_{m} \mathbf{V}_{i}^{*}\right\|^{2}-\mathcal{I}_{m}\right) \\
=\left\|\mathbf{V}^{*}\right\|^{2}+\sum_{m} \lambda_{m}\left(\left\|\mathbf{H}_{m} \mathbf{V}_{m}^{*}-\mathbf{G}_{m} \mathbf{W}_{m}\right\|^{2}+\sum_{i \neq m}\left\|\mathbf{H}_{m} \mathbf{V}_{i}^{*}\right\|^{2}-\mathcal{I}_{m}\right) \\
\quad+\sum_{m}\left(\lambda_{m}^{\prime}-\lambda_{m}\right)\left(\left\|\mathbf{H}_{m} \mathbf{V}_{m}^{*}-\mathbf{G}_{m} \mathbf{W}_{m}\right\|^{2}+\sum_{i \neq m}\left\|\mathbf{H}_{m} \mathbf{V}_{i}^{*}\right\|^{2}-\mathcal{I}_{m}\right) \\
=g(\boldsymbol{\lambda})+\sum_{m}\left(\lambda_{m}^{\prime}-\lambda_{m}\right)\left(\left\|\mathbf{H}_{m} \mathbf{V}_{m}^{*}-\mathbf{G}_{m} \mathbf{W}_{m}\right\|^{2}+\sum_{i \neq m}\left\|\mathbf{H}_{m} \mathbf{V}_{i}^{*}\right\|^{2}-\mathcal{I}_{m}\right),
\end{gathered}
$$

where $\mathbf{V}^{*}$ is the solution derived form equation (2.19) for $\boldsymbol{\lambda}$. Now if we consider

$$
\begin{aligned}
\mathbf{d} & =\left(d_{1}, \ldots, d_{M}\right)^{T} \\
d_{i} & =\left(\left\|\mathbf{H}_{m} \mathbf{V}_{m}^{*}-\mathbf{G}_{m} \mathbf{W}_{m}\right\|^{2}+\sum_{i \neq m}\left\|\mathbf{H}_{m} \mathbf{V}_{i}^{*}\right\|^{2}\right),
\end{aligned}
$$

then the inequality

$$
g\left(\boldsymbol{\lambda}^{\prime}\right) \leq g(\boldsymbol{\lambda})+\left(\boldsymbol{\lambda}^{\prime}-\boldsymbol{\lambda}\right)^{T} \mathbf{d}
$$

shows that $\mathbf{d}$ is sub-gradient by definition.

Finally, we invoke the sub-gradient method to solve optimization problem (2.22) by using the following iterations:

$$
\boldsymbol{\lambda}^{j+1}=\boldsymbol{\lambda}^{j}+\alpha_{k} \mathbf{d},
$$

where $\alpha_{k}>0$ is the $k$ th step size.

The proposed algorithm is summarized below. 


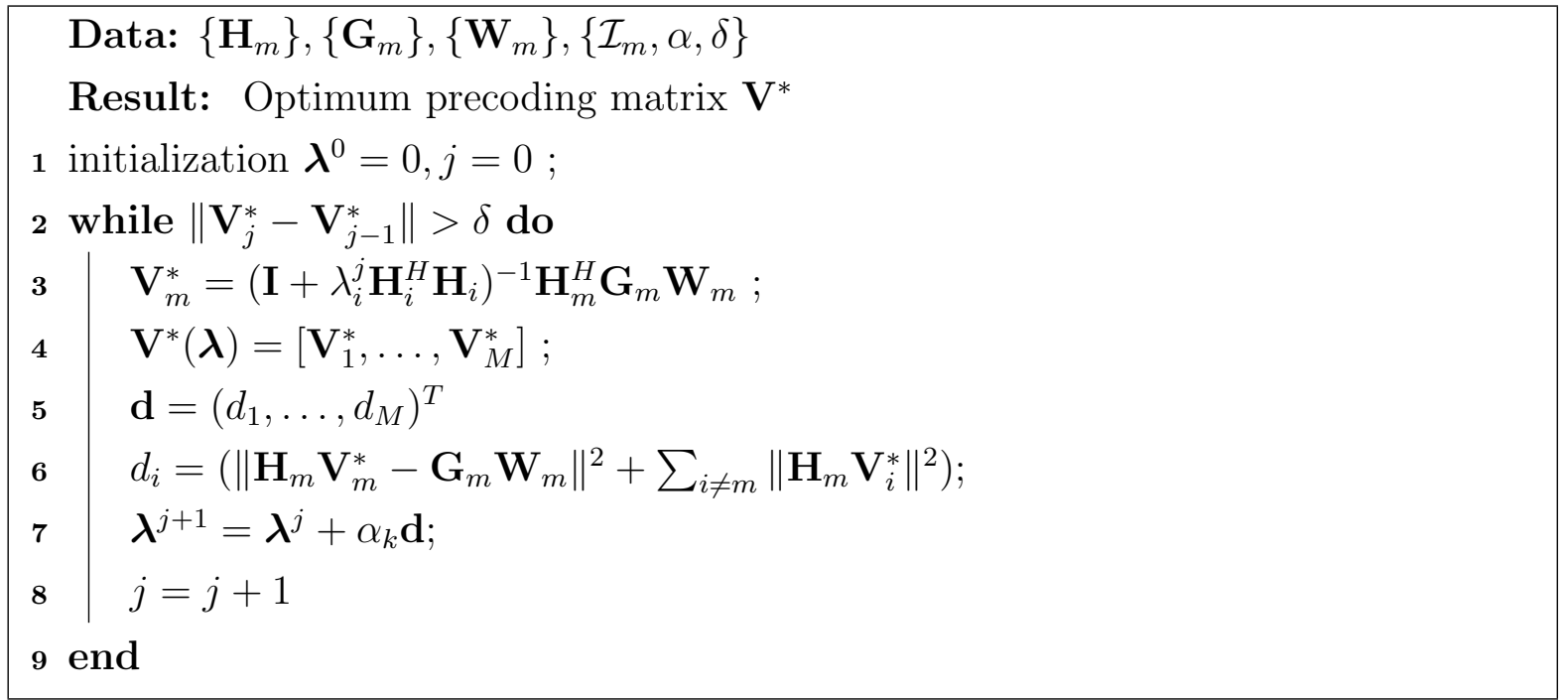

Algorithm 1: Minimizing transmission power subject to constraints for the SPS for Perfect CSI

\subsection{Power Minimization for Non-perfect CSI}

\subsubsection{Problem Statement}

In the previous section, we assume that the channel is perfectly known at the InP. However, this assumption is far from reality due to factors such as channel estimation error and pilot contamination. Since we initially consider a single-cell network in our model, we only take into account the error caused by channel estimation. However, it does not limit our derivations since considering other factors such as pilot contamination only changes the covariance matrix of the error.

We adopt the model

$$
\mathbf{H}_{m}=\hat{\mathbf{H}}_{m}+\mathbf{E}_{m}
$$

for the channel where $\mathbf{H}_{m}$ is the unknown true channel of user of SP $m, \hat{\mathbf{H}}_{m}$ is the estimated channel, and $\mathbf{E}_{m}$ is the channel estimation error such that $\operatorname{vec}\left(\mathbf{E}_{m}\right)=\mathcal{C N}\left(\mathbf{0}, \mathbf{C}_{e}\right)$.

There are two possible approaches to deal with the channel uncertainty:

- The first approach is to formulate the problem in the worst case. For the problem under consideration, it is formulated as

$$
\begin{array}{cl}
\min _{\mathbf{V}} & \|\mathbf{V}\|^{2} \\
\text { subject to: } & f_{m}(\mathbf{V}) \leq \mathcal{I}_{m}^{2} \quad \forall m
\end{array}
$$


where

$$
\begin{aligned}
f_{m}(\mathbf{V}) & =\max _{\mathbf{E}_{m} \in \mathcal{C}_{m}}\left\|\mathbf{H}_{m} \mathbf{V}_{m}-\mathbf{G}_{m} \mathbf{W}_{m}\right\|^{2}+\sum_{i \neq m}\left\|\mathbf{H}_{m} \mathbf{V}_{i}\right\|^{2} \\
& =\max _{\mathbf{E}_{m} \in \mathcal{C}_{m}}\left\|\left(\hat{\mathbf{H}}_{m}+\mathbf{E}_{m}\right) \mathbf{V}_{m}-\mathbf{G}_{m} \mathbf{W}_{m}\right\|^{2}+\sum_{i \neq m}\left\|\mathbf{H}_{m} \mathbf{V}_{i}\right\|^{2},
\end{aligned}
$$

and $\mathcal{C}_{m}$ is the set of values taken by $\mathbf{E}_{m}$. Optimization problem (2.35-2.36) has another optimization problem inside, which is problem (2.38). It can be proved that the former is convex and the latter has a solution occurs at the boundaries and can be found by duality.

- The second approach is to formulate the problem with chance constraints. For our purpose, this formulations yields the following problem:

$$
\begin{aligned}
\min _{\mathbf{V}} & \|\mathbf{V}\|^{2} \\
\text { subject to: } & \mathbb{P}\left\{\left\|\mathbf{H}_{m} \mathbf{V}_{m}-\mathbf{G}_{m} \mathbf{W}_{m}\right\|^{2}+\sum_{i \neq m}\left\|\mathbf{H}_{m} \mathbf{V}_{i}\right\|^{2} \leq \mathcal{I}_{m}^{2}\right\} \geq 1-\epsilon
\end{aligned}
$$

We adopt the second approach due to its popularity and complications.

\subsubsection{Proposed Solution for Non-Perfect CSI}

Generally, optimization problems with chance constraints are hard to solve and simple solution does not usually exist. However, in the following we present a solution to this problem that is not computationally cumbersome and will be numerically shown has close to optimal performance.

To solve (2.39-2.40), we first present a lemma which provides a formula to derive the probability in (2.40). We later use this lemma to check the feasibility of solutions. We then propose a lower bound for problem (2.57-2.58), and finally describe our proposed solution.

Lemma 2.3.1. Given a Hermitian matrix $\mathbf{A}$ and complex vector $\mathbf{b}$, for circular complex Gaussian random vector $\mathbf{e}$ distributed as $\mathcal{C N}\left(\mathbf{0}, \mathbf{C}_{e}\right)$, the $C D F$ of $\|\mathbf{A e}+\mathbf{b}\|^{2}$ is given by

$$
\mathbf{P}\left(\|\mathbf{A e}+\mathbf{b}\|^{2} \leq \tau\right)=\int_{-\infty}^{\infty} \frac{e^{\tau(\beta+j \omega)}}{(\beta+j \omega)} \frac{e^{-c_{0}(\omega)+c(\omega)}}{\prod_{i}\left(1+(\beta+j \omega) D_{i}^{2}\right)} d \omega
$$


where

$$
\begin{aligned}
& c_{0}(\omega)=(\beta+j \omega) \mathbf{b}^{H} \mathbf{b} \\
& c(\omega)=(\beta+j \omega)^{2} \sum_{i} \frac{\left|a_{i}\right|^{2} D_{i}^{2}}{1+(\beta+j \omega) D_{i}^{2}} \\
& \mathbf{a}=\mathbf{U}^{H} \mathbf{b} \\
& \mathbf{U D Q}=\operatorname{svd}\left(\mathbf{A C}_{e}\right)
\end{aligned}
$$

for some $\beta>0$.

Remark. This lemma was inspired by [26] in which the authors provided a closed-form expression for the CDF of $\|\mathbf{e}-\mathbf{b}\|_{\mathbf{Q}}^{2}$ for a Hermitian matrix $\mathbf{Q}$. We followed a slightly different approach to obtain the CDF of $\|\mathbf{A e}+\mathbf{b}\|^{2}$.

Proof. The proof is provided in Appendix A.2.

To invoke this lemma for our purpose, we transform (2.11) to vector form as follows:

$$
\begin{aligned}
& \left\|\mathbf{H}_{m} \mathbf{V}_{m}-\mathbf{G}_{m} \mathbf{W}_{m}\right\|^{2}+\sum_{i \neq m}\left\|\mathbf{H}_{m} \mathbf{V}_{i}\right\|^{2} \\
= & \left\|\mathbf{H}_{m} \mathbf{V}-\mathbf{G}_{m}\left[\mathbf{0}, \ldots, \mathbf{W}_{m}, \ldots, \mathbf{0}\right]\right\|^{2} \\
= & \left\|\left(\hat{\mathbf{H}}_{m}+\mathbf{E}_{m}\right) \mathbf{V}-\mathbf{G}_{m}\left[\mathbf{0}, \ldots, \mathbf{W}_{m}, \ldots, \mathbf{0}\right]\right\|^{2} \\
= & \left\|\mathbf{E}_{m} \mathbf{V}+\hat{\mathbf{H}}_{m} \mathbf{V}-\mathbf{G}_{m}\left[\mathbf{0}, \ldots, \mathbf{W}_{m}, \ldots, \mathbf{0}\right]\right\|^{2} \\
= & \|\mathbf{A} \mathbf{e}+\mathbf{b}\|^{2}
\end{aligned}
$$

where

$$
\begin{aligned}
& \mathbf{A}=\mathbf{V}^{T} \otimes \mathbf{I}_{K_{m}} \\
& \mathbf{e}_{m}=\operatorname{vec}\left(\mathbf{E}_{m}\right) \\
& \mathbf{b}_{m}=\operatorname{vec}\left(\hat{\mathbf{H}}_{m} \mathbf{V}-\mathbf{G}_{m}\left[\mathbf{0}, \ldots, \mathbf{W}_{m}, \ldots, \mathbf{0}\right]\right) .
\end{aligned}
$$

Now we turn to describing the lower bound for problem (2.39-2.40). In Appendix A.3, we showed that (2.11) can be written as the sum of $K N$ independent normal random variables. Assuming this sum converges to a normally distributed random variable and since for a normally distributed random variable the following property holds(Proof in 
Appendix A.4): For $\epsilon \leq 0.5$,

$$
\mathbb{P}\left\{\left\|\mathbf{H}_{m} \mathbf{V}-\mathbf{G}_{m}\left[\mathbf{0}, \ldots, \mathbf{W}_{m}, \ldots, \mathbf{0}\right]^{2}\right\| \leq \mathcal{I}_{m}^{2}\right\} \geq 1-\epsilon
$$

implies

$$
\mathbb{E}\left\|\mathbf{H}_{m} \mathbf{V}-\mathbf{G}_{m}\left[\mathbf{0}, \ldots, \mathbf{W}_{m}, \ldots, \mathbf{0}\right]\right\|^{2} \leq \mathcal{I}_{m}^{2}
$$

we can say the convex optimization problem given by

$$
\begin{aligned}
\min _{\mathbf{V}} & \|\mathbf{V}\| \\
\text { subject to: } & \mathbb{E}\left\|\mathbf{H}_{m} \mathbf{V}-\mathbf{G}_{m}\left[\mathbf{0}, \ldots, \mathbf{W}_{m}, \ldots, \mathbf{0}\right]\right\|^{2} \leq \mathcal{I}_{m}^{2}
\end{aligned}
$$

provides a lower bound to the optimization problem defined in (2.39-2.40). This lower bound will be used to design the proposed algorithm and also to evaluate the performance of the algorithm.

The expected value in (2.56) can be obtained as follows and the proof is provided in Appendix A.5.

$$
\mathbb{E}\left\|\mathbf{H}_{m} \mathbf{V}-\left[\mathbf{0}, \ldots, \mathbf{W}_{m}, \ldots, \mathbf{0}\right]\right\|^{2}=\|\mathbf{D V}\|^{2}+\left\|\hat{\mathbf{H}}_{m} \mathbf{V}-\left[\mathbf{0}, \ldots, \mathbf{W}_{m}, \ldots, \mathbf{0}\right]\right\|^{2}
$$

where $\mathbf{D}$ is a diagonal matrix, $\operatorname{diag}\left(D_{1}, \ldots, D_{N}\right)$, where

$$
D_{i}=\sqrt{\sum_{i} \sigma_{i, j}^{2}}
$$

Finally in what follows, we describe our algorithm for solving optimization problem (2.39-2.40).

The proposed solution is based on the lower bound provided above. We describe the concept behind the algorithm using Figure 2.2. Assume the shaded area, $S$, is the feasibility set of problem (2.39-2.40) and let us define the following optimization problem for each $\theta$ :

$$
\begin{aligned}
& P_{\theta}: \min _{\mathbf{V}}\|\mathbf{V}\| \\
& \text { subject to: } \quad \mathbb{E}\left\|\mathbf{H}_{m} \mathbf{V}-\mathbf{G}_{m} \mathbf{W}_{m}\right\|^{2} \leq(\theta \mathcal{I})_{m}^{2} \text {. }
\end{aligned}
$$

Areas $S_{\theta}$ corresponds to the feasibility set of $P_{\theta}$. As previously mentioned, $S_{1} \subset S$. At 


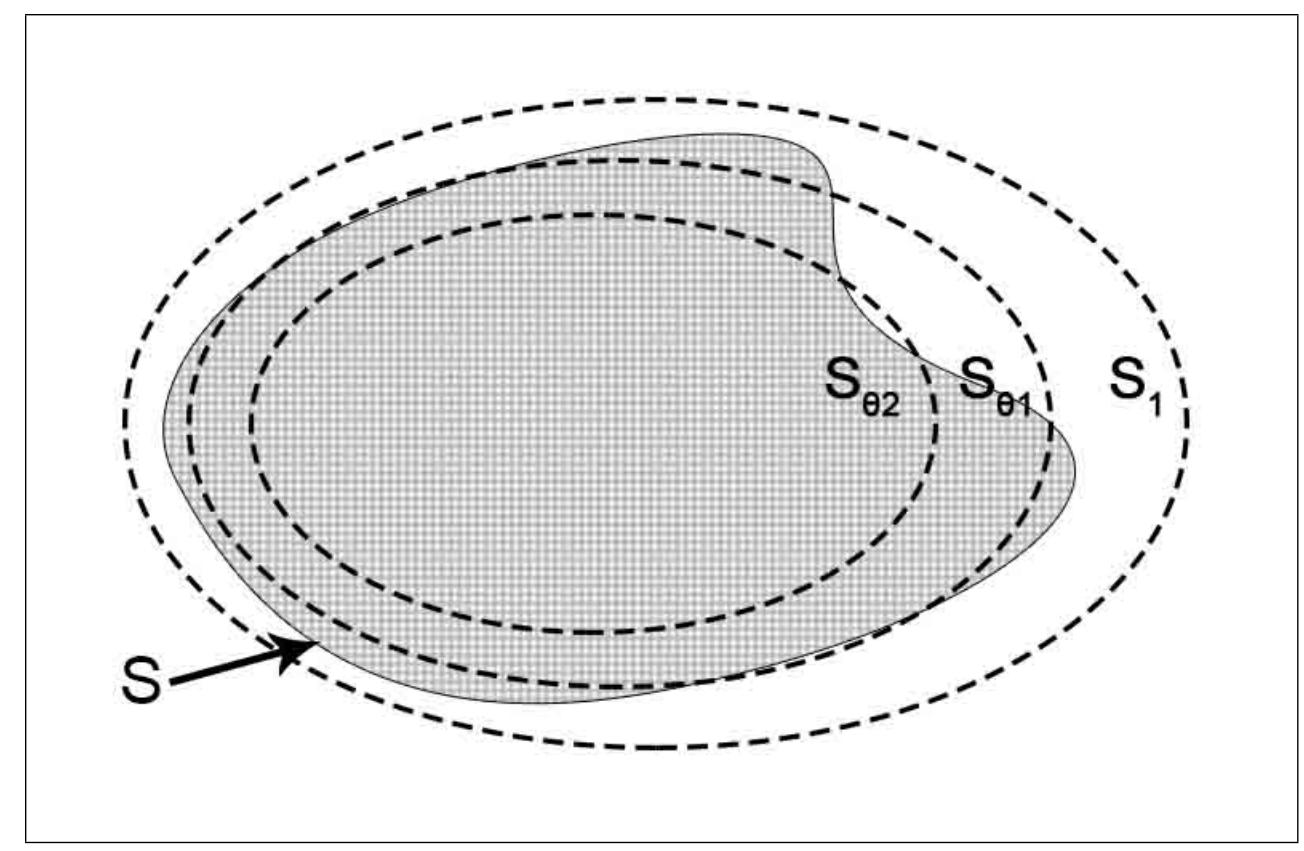

Figure 2.2: A picture of feasible sets of the problems

each iteration, we shrink the feasibility set of $P_{\theta}$ by decreasing $\theta$ and solve $P_{\theta}$. Then the solution to $P_{\theta}$ is checked by using the formula given in (2.41) to know whether it resides in $S$. If so, the algorithm ends, otherwise $S_{\theta}$ is shrunk further to hopefully find a solution residing in $S$. This procedure continues until either a solution is found or $P_{\theta}$ becomes infeasible. It should be noted that $P_{\theta}$ is a convex programming problem and hence can be solved in polynomial time.

The proposed algorithm is summarized below. We used notations $\mathbf{V}_{\theta}$ and $\alpha$ to respectively denote the solution to $P_{\theta}$ and algorithm step size. 


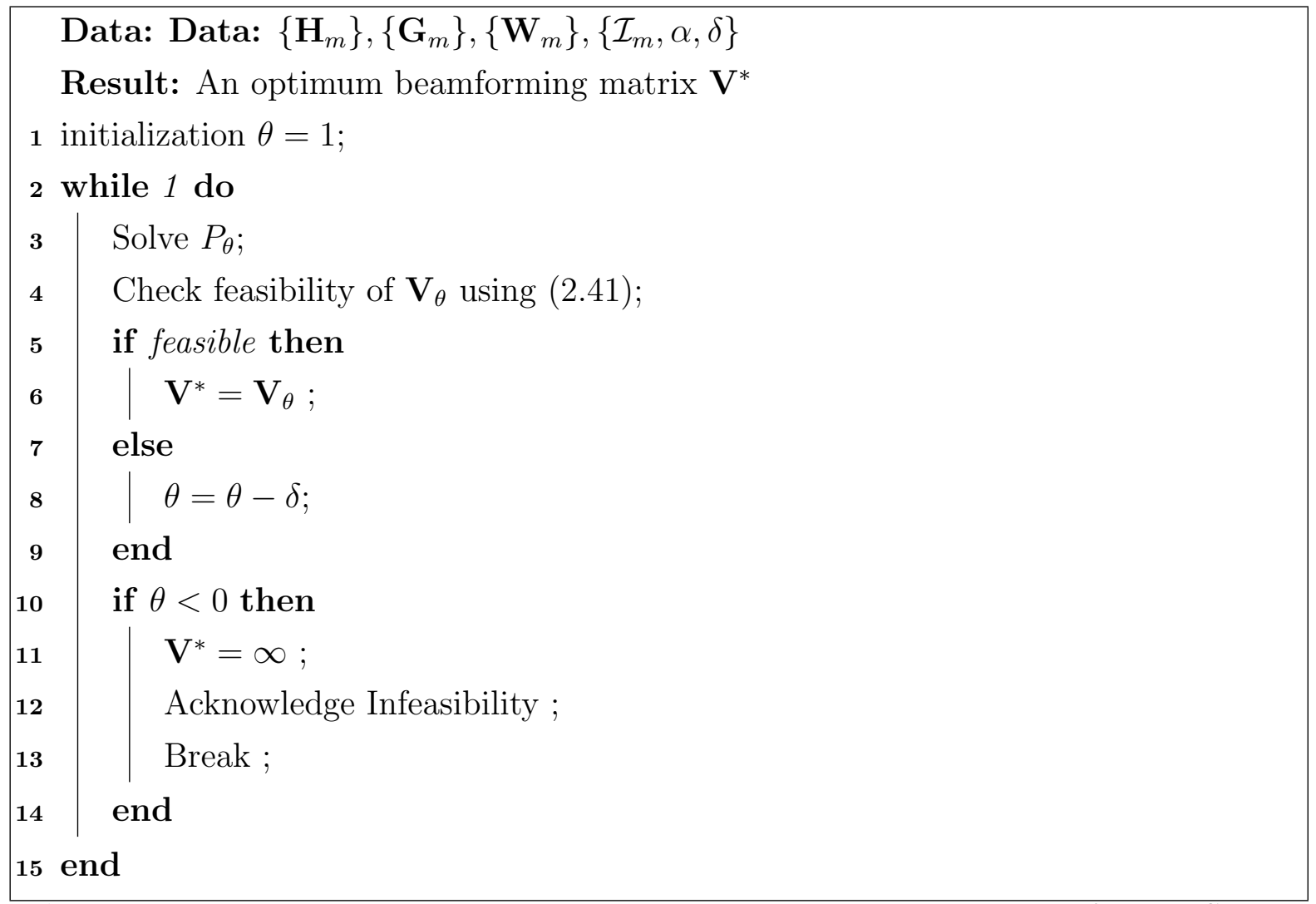

Algorithm 2: Minimizing transmission power subject to constraints for the SPs for imperfect CSI

\subsection{Three Extensions}

In this section, we introduce three extensions to the precoding problem and show that these extensions can be readily resided to our current formulation.

\subsubsection{Adding SINR constraints}

In addition to serving the SPs, the InP may have a set of users that are to be served by the InP. Thus, the InP should consider a set of QoS constraints for these users as well as the performance constraints for the SPs. Adding these QoS constraints to the optimization problems leads to a new optimization problem which is potentially complicated. For instance, if we write the QoS constraints of users in terms of SINR, the problem can be 
formulated as follows.

$$
\begin{aligned}
\min _{\mathbf{V}} & \|\mathbf{V}\|^{2} \\
\text { subject to: } & \left\|\mathbf{H}_{m} \mathbf{V}_{m}-\mathbf{G}_{m} \mathbf{W}_{m}\right\|^{2}+\sum_{i \neq m}\left\|\mathbf{H}_{m} \mathbf{V}_{i}\right\|^{2} \leq \mathcal{I}_{m}^{2} \quad \forall m \\
& \operatorname{SINR}_{k} \geq \gamma_{k} \quad k \in \mathcal{K}_{0}
\end{aligned}
$$

where $\mathcal{K}_{0}$ is the set of users of the InP. This problem is non-convex. One may be able to relax it solve it using SOCP, but it is difficult to evaluate the performance of such solution approach.

We approach this problem by considering the InP itself as a new SP, call it SP0. This SP0 designs a precoding matrix for its users based on traditional precoding schemes to guarantee the QoS of its users. The InP can then easily solve the optimization given in (2.13-2.14) or (2.39-2.40), but with one more SP.

\subsubsection{Mitigating Inter-cell Interference}

We modelled the precoding problem for a single-cell cellular network. In this section we show multi-cell network can also work with our scheme.

In a multicell-network, the BS maintains the interference to the neighbouring cells under a certain threshold. Let $\mathbf{H}_{0}$ be the channel between the BS and the users of neighbouring cells. The interference to these users should be below a certain value, or mathematically,

$$
\left\|\mathbf{H}_{0} \mathbf{V}\right\|_{F} \leq \mathcal{I}_{0}
$$

This constraint has the same form as the constraints for the SP but with $\mathbf{W}_{m}=0$. Therefore, adding constraints to suppress the interference in the neighbouring cells leads to an optimization problem with the same form as (2.13-2.14) or (2.39-2.40), but with more constrains.

\subsubsection{Per User Constraint}

From the constraints in (2.13-2.14) or (2.39-2.40), it can be inferred that the constraints ensure that the total sum of the deviations of all users of each SP is guaranteed to be below a given value. However, it does not necessarily impose a limit on the deviation of a single user. The $\mathrm{InP}$ can customize its service to the SPs by allowing them to also set the 
deviation limit per user, which means the InP should satisfy the following constraints:

$$
\mathbb{E}_{\mathbf{x}}\left\|\mathbf{h}_{m}^{k} \mathbf{V} \mathbf{x}-\mathbf{g}_{m}^{k} \mathbf{W}_{m} \mathbf{x}_{m}\right\|_{F}^{2} \leq \mathcal{I}_{m}^{k} \quad \forall k \in \tilde{K}_{m}
$$

where $\mathbf{h}_{m}^{k}$ is the channel of $k$ th users of SP $m$, and $\tilde{K}_{m}$ is the set of target users of SP $m$.

These constraints have the same form as the constraints in problem (2.13-2.14) or (2.39-2.40). Thus, the same approach can be adopted to solve this optimization problem.

\subsection{Antenna Selection}

Thus far we focused on designing a precoding given the set of antennas to be communicated to the SPs, and to perform the transmission are known. In this section we propose algorithms for each of these selections.

\subsubsection{Antenna Selection in Step 1}

At each precoding time slot and for each SP $m$, the InP selects a subset of antennas and provides the SP with the corresponding channel information. Again, assume that the InP's BS is equipped with $N$ antennas and it aims to select the best $N_{m}$ antennas for SP $m$. Since the InP does not know neither the precoding scheme of the SP nor the their preferences, it resorts to capacity of MIMO system as objective. It is given by

$$
\left.C=\log \operatorname{det}\left(\mathbf{I}+\rho \mathbf{H}_{m} \mathbf{H}_{m}^{H}\right)\right) .
$$

So, the InP solves the optimization problem defined by

$$
\begin{aligned}
\max _{\boldsymbol{\Delta}} & \left.\log \operatorname{det}\left(\mathbf{I}+\rho \mathbf{H}_{m} \boldsymbol{\Delta} \mathbf{H}_{m}^{H}\right)\right) \\
\text { subject to: } & \Delta_{i} \in\{0,1\}
\end{aligned}
$$

where $\boldsymbol{\Delta}$ is a diagonal matrix with diagonal entries being $\left(\Delta_{1}, \ldots, \Delta_{N}\right)$.

This combinatorial optimization problem has been already studied and several approaches have been proposed. One solution is to relax the constraint (2.70) to

$$
\Delta_{i} \in[0,1]
$$

and then the problem is relaxed to a convex programming. After solving the problem, we can extract a solution by choosing the $N_{m}$ largest entries of $\boldsymbol{\Delta}$. 


\subsubsection{Antenna Selection in Step 3}

After the SPs design the precoding matrices, the InP selects a subset of antennas to perform the transmission. One motivation of using a subset of antennas rather than all can be saving power by turning off the RF-chain circuit behind the antennas. So we take power minimization as the objective of the InP and assume that power consumption per RF-chain circuit is $P_{\mathrm{RF}}$. Define the following optimization problem

$$
\begin{array}{cl}
\min _{\mathbf{V}, \boldsymbol{\Delta}} & \sum_{s \in \mathcal{S}}\left\|\mathbf{V}^{s}\right\|^{2}+P_{\mathrm{RF}} \operatorname{Trace}(\boldsymbol{\Delta}) \\
\text { subject to: } & \left\|\mathbf{H}_{m}^{s} \Delta \mathbf{V}_{m}^{s}-\mathbf{G}_{m}^{s} \mathbf{W}_{m}^{s}\right\| \leq \mathcal{I}_{m}^{s} \forall m, s
\end{array}
$$

where superscript $s$ refer to sub-carriers $s$ and $\mathcal{S}$ is the set of all sub-carriers. This is a mixed-combinatorial problem and finding the optimal solution imposes a computational complexity on the InP. Nevertheless, we propose the following greedy algorithm to select a subset of antennas, which is not computationally cumbersome and can potentially help in saving power.

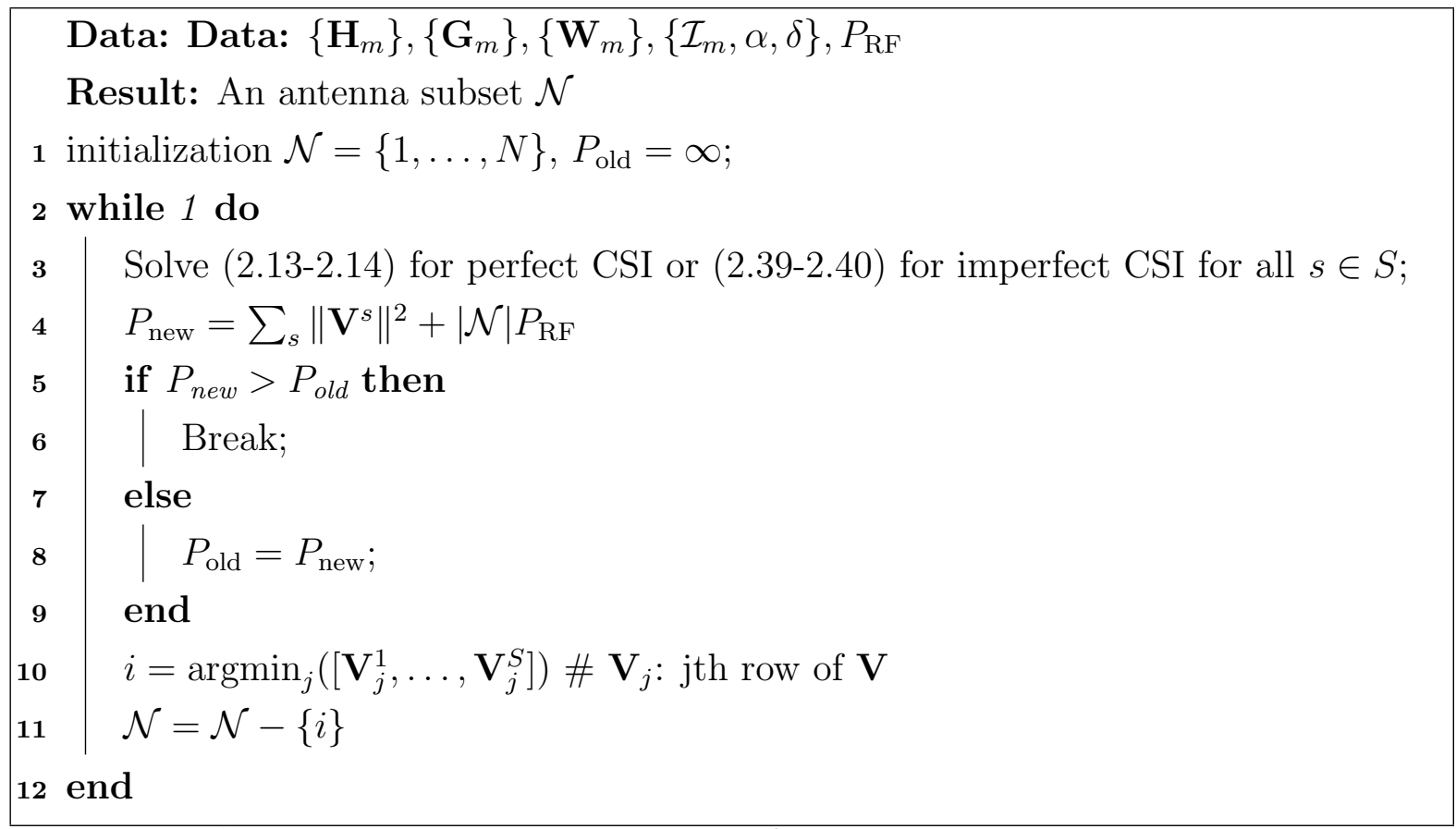

Algorithm 3: Finding a subset of antennas to save power

At each iteration, the algorithm turns off the antenna that corresponds to the row of all $\mathbf{V}$ combined that has the smallest norm, which is the antenna that has the smallest contribution in satisfying the constraint, and continue until turning off an antenna does not decrease total required power. 


\section{Chapter 3}

\section{Simulations and Performance Evaluation}

In this chapter, we present the simulation results for our proposed algorithms and numerically study the performance of precoding when perfect channel information is available and reason why InP may prefer to provide a subset of antennas to the SPs. We also numerically study the performance of precoding when the channel information is corrupted with channel estimation noise. We show that our proposed algorithm performs close to optimal. Finally, we present the simulation results for the proposed antenna selection algorithms.

\subsection{Simulation Settings}

Unless otherwise stated, we consider a hexagon-cell with a base station at its center, as shown in Figure 3.1. The users of the SPs are uniformly distributed across the cell. Different colors in the figure represent users of different SPs.

We model the baseband fading channel that links the base station to the $k$ th user by

$$
\begin{aligned}
& \mathbf{h}_{k}=\beta_{k}^{1 / 2} \mathbf{g}_{k} \quad \forall k \\
& \beta_{k}[\mathrm{~dB}]=-31.54-37.1 \log _{10}\left(d_{k}\right)-8 \psi_{k} \quad \forall k
\end{aligned}
$$

where $\mathbf{g}_{k}$, distributed as $\mathcal{C N}\left(\mathbf{0}, \mathbf{I}_{N}\right)$, model small scale fading, $\beta_{k}$ capture large scale fading including path loss and shadowing effects, $d_{k}$ is the distance of user $k$ from the BS, and $\psi_{k}$ is standard Gaussian random variable which accounts for large-scale shadowing.

As previously mentioned, the virtualization is performed in three steps: 


\begin{tabular}{|l|c|}
\hline Parameter & Value \\
\hline$P$ & $8 \mathrm{dBm}$ \\
\hline$\sigma_{n}^{2}$ & $-174 \mathrm{dBm} / \mathrm{W}$ \\
\hline $\mathrm{BW}$ & $10 \mathrm{KHz}$ \\
\hline$R_{c}$ & $500 \mathrm{~m}$ \\
\hline
\end{tabular}

Table 3.1: Initial simulation parameters

- In Step 1, The InP communicates the channel information of the antennas to the SPs. Unless otherwise stated, we assume the InP provides the channel information of all antennas to the SPs.

- In Step 2, the SPs design precoding matrices $\mathbf{W}_{m}$. We assume that Regularized Zero Forcing (RZF) precoding is employed by the SPs. This precoding is obtained by

$$
\mathbf{W}_{m}=\mathbf{G}_{m}^{H}\left(\mathbf{G}_{m} \mathbf{G}_{m}^{H}+\frac{K_{m}}{\sigma_{n}^{2}} \mathbf{I}\right)^{-1}
$$

where $\sigma_{n}$ is the noise power and is normalized to

$$
\left\|\mathbf{W}_{m}\right\|=\frac{P}{\sqrt{M}}
$$

and $P$ the amount of power that the InP dedicates to all the SPs.

- In Step 3, the InP designs the precoding matrix by the algorithm proposed in Section 2.3.2. We define deviation factors, $\rho_{m}$, and use them to set values of $\mathcal{I}_{m}$,i.e.,

$$
\mathcal{I}_{m}=\rho_{m}\left\|\mathbf{H}_{m} \mathbf{W}_{m}\right\|
$$

The deviation factors can be interpreted as how much relatively the InP's precoding is allowed to deviate from the SP's precoding. To reduce the number of system variables, we assume $\rho_{m}=\rho$ for $m=1, \ldots, M$.

For our simulation, we usually change a system parameter and keep the other system's parameters to their default value. Table 3.1 displays these default values that are used throughout our simulations. 


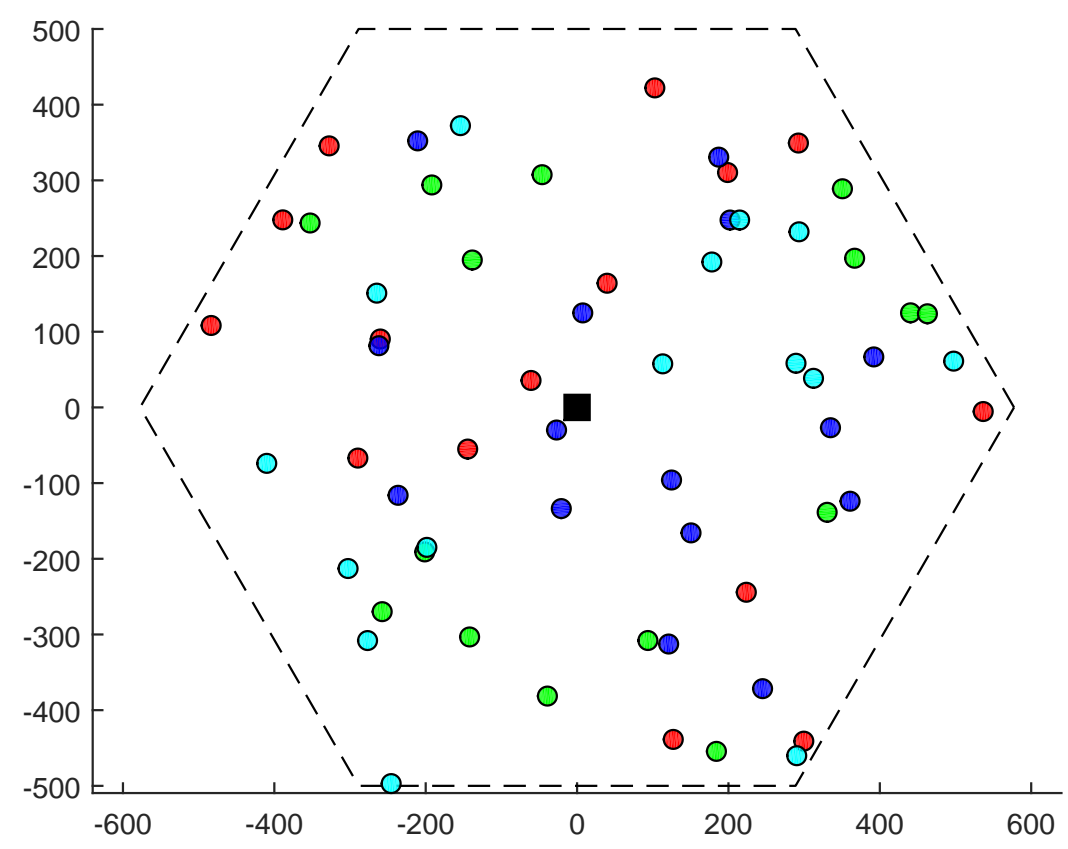

Figure 3.1: A realization of a cell with 4 SPs that each has 15 users. Different colours represent users of different SPs.

\begin{tabular}{|l|c|}
\hline Parameter & Value \\
\hline$M$ & 4 \\
\hline$K_{m}$ & 15 \\
\hline$N$ & 100 \\
\hline$N_{m}$ & 100 \\
\hline$\rho$ & 0.1 \\
\hline
\end{tabular}

Table 3.2: Default simulation parameters for perfect CSI

\subsection{Numerical Results for Precoding with Perfect CSI}

We consider a network consists of 4 SPs, each with 15 users, and an InP with a base station that is equipped with 100 antennas (Table 3.2).

Figure 3.2 plots the received SINR of users of the SPs. Different colours represent the users of different SPs and each line corresponds to a user. The sub-plot at the top displays the SPs desired received signals, which is determined by the given precoding, $\mathbf{W}_{m}$, and the next three figures display the actual received SINR for different values of $\rho$, resulted from the precoding matrix designed by the InP. As can be seen in these plots, 
as $\rho$ decreases, the actual received signals of the users converge to the SPs' designs. The SPs prefer smaller values for $\rho$ as it makes the actual received signals resemble more the SP's precoding. The InP can satisfy whatever smaller value of $\rho$ but at the expense of more transmission power. Figure 3.3 displays the norm of $\mathbf{V}$, or the square root of transmission power, with respect to deviation factor $\rho$ for different values of $N$. As this figure demonstrates, the InP should use more transmission power to satisfy the constraints with smaller $\rho$.

Figure 3.4 displays norm of $\mathbf{V}$ with respect to the number of transmission antennas. Each curve corresponds to a given $\rho$. As can be seen, from a point, increasing the number of antenna does not noticeably impact the transmission power. This motivates the idea to use fewer antennas for transmission in order to save power by turning off the RF-chain circuits behind them.

\subsection{Numerical Results for Precoding with Imperfect CSI Precoding}

In our simulations, we assume the CSI is corrupted due to not perfect channel estimation. We have simulated the channel estimation procedure to obtain the imperfect CSI. This procedure is briefly described in the next subsection.

\subsubsection{Channel Estimation through Uplink Training}

For regular MIMO systems, multi-user precoding in the downlink and detection in the uplink require CSI at the BS. The resource, time or frequency, required for channel estimation in a MIMO system is proportional to the number of the transmit antennas and is independent of the number of users and the receive antennas.

If FDD is used, to get CSI for the downlink channel, a two-stage procedure is required. The BS first transmits pilot symbols to all users, and then all users feed back estimated CSI (partial or complete) for the downlink channels to the BS. The time required to transmit the downlink pilot symbols is proportional to the number of antennas at the BS. As the number of BS's antennas grows large, the traditional downlink channel estimation strategy for FDD systems becomes infeasible. For example, consider a $1 \mathrm{~ms} 100 \mathrm{kHz}$ channel coherence interval, which can support transmission of 100 complex symbols. When there are 100 antennas at the BS, the whole coherence interval will be used for downlink training if orthogonal pilot waveforms are used for channels to each antenna, while there is no symbol left for data transmission. Fortunately, the channel estimation 


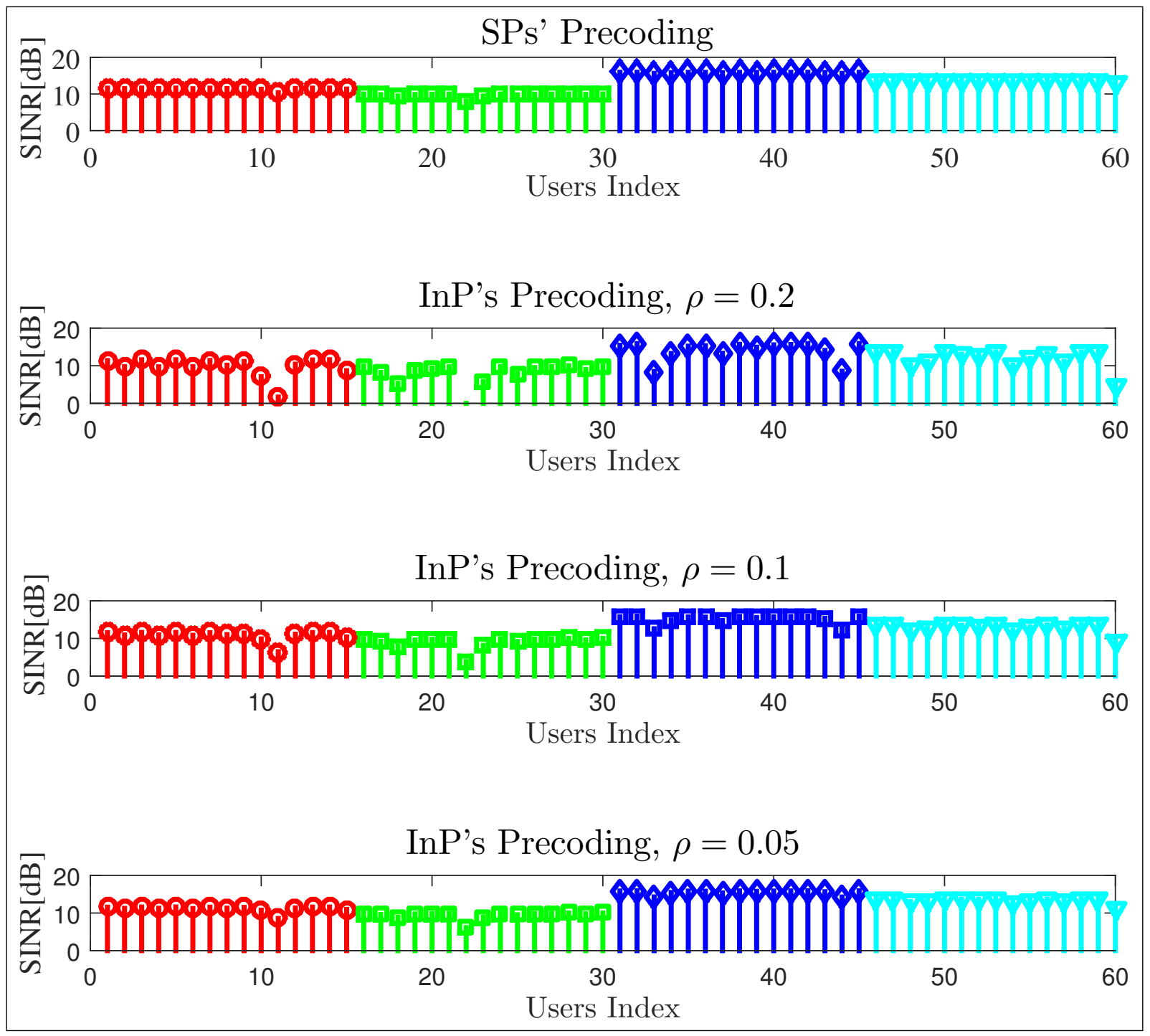

Figure 3.2: Users' received SINR for different values of $\rho$ and SPs precoding 


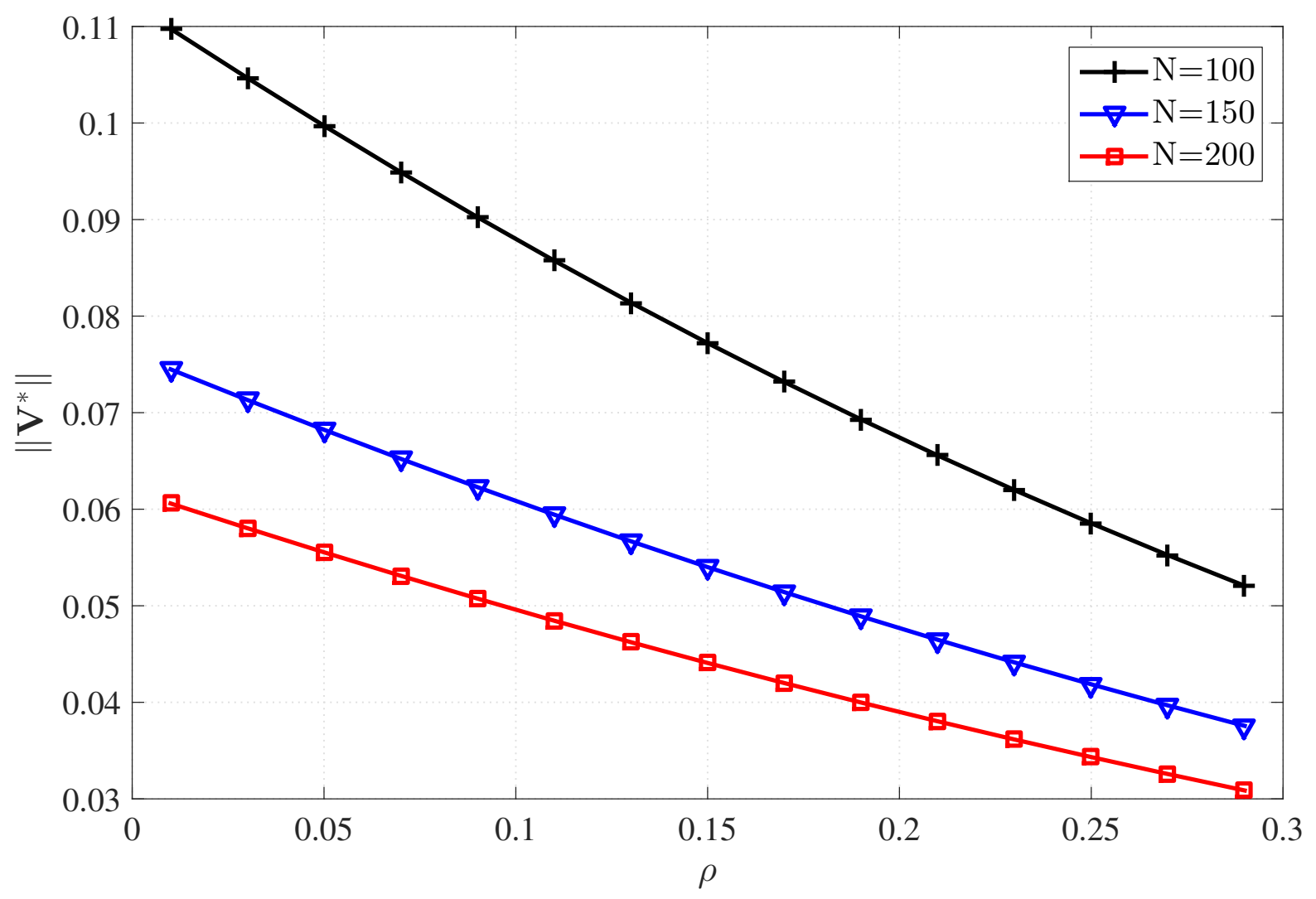

Figure 3.3: Norm of $\mathbf{V}^{*}$ vs $\rho$ for different number of antennas 


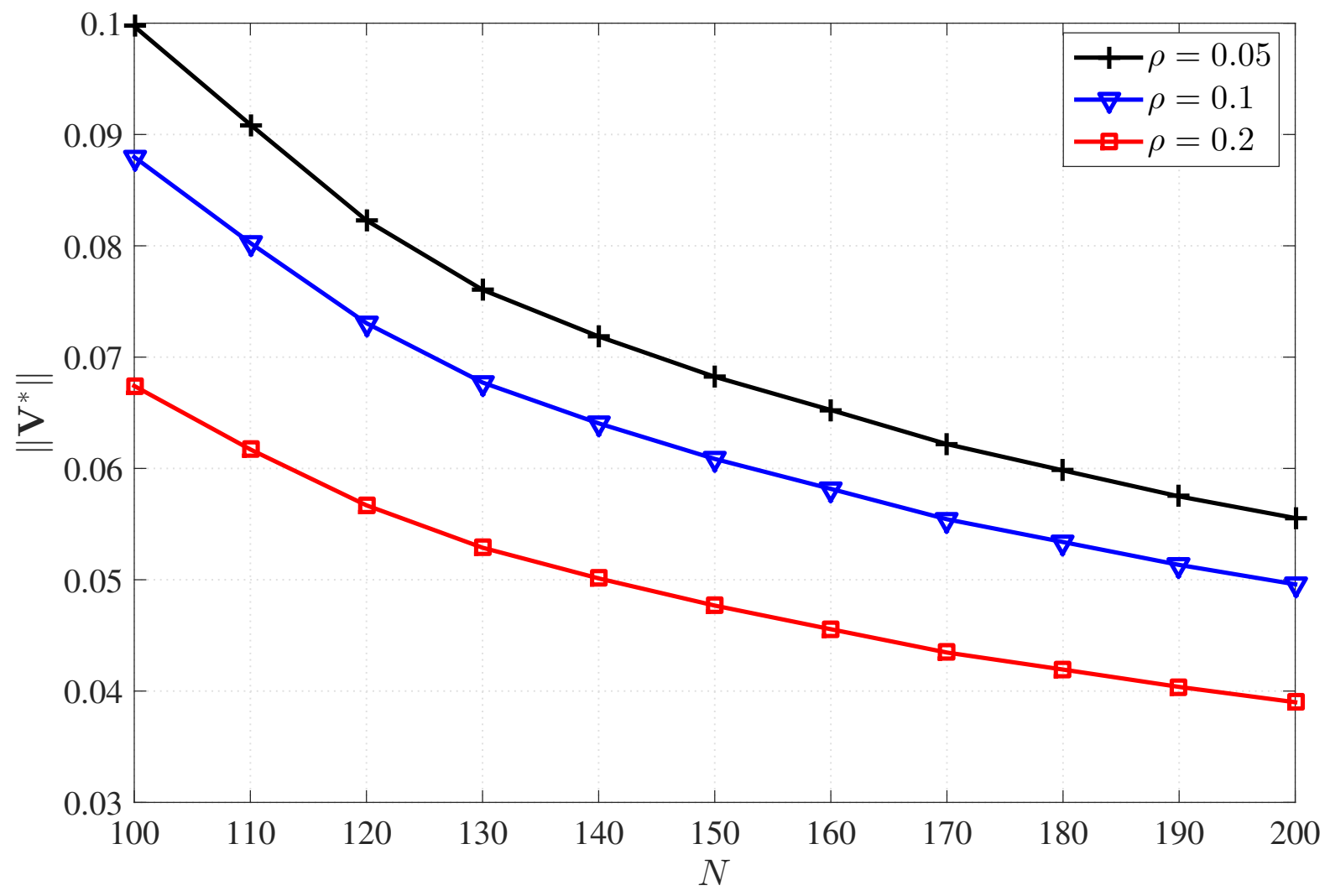

Figure 3.4: Norm of $\mathbf{V}^{*}$ vs $N$ for different values of $\rho$ 
strategy in TDD systems can be utilized to solve the problem. Based on the assumption of channel reciprocity, only CSI for the uplink needs to be estimated.

Suppose a TDD system and assume that channel reciprocity holds. One way to estimate the channel is to have the user sent $L$ pilot symbols one by one with power $P^{\mathrm{ul}}$. Let $\mathbf{y}_{k}^{i}$ be the $i$ th received pilot of user $k$ at the BS which is obtained by

$$
\mathbf{y}_{k}^{i}=\sqrt{P^{\mathrm{ul}}} \mathbf{h}_{k}+\mathbf{n}
$$

where $\mathbf{n} \sim \mathcal{C N}\left(0, \sigma_{n}^{2}\right)$ is the noise at the receiver. The minimum mean square error (MMSE) estimate, $\hat{\mathbf{h}}_{k}$, of the channel $\mathbf{h}_{k}$ is obtained by

$$
\hat{\mathbf{h}}_{k}=\mathbf{C}_{\mathbf{h}_{k}}^{T} \mathbf{T}^{T}\left(\mathbf{T C}_{\mathbf{h}_{k}}^{T} \mathbf{T}^{T}+\sigma_{n}^{2} \mathbf{I}\right)^{-1}\left[\mathbf{y}_{k}^{1} ; \ldots ; \mathbf{y}_{k}^{T}\right]
$$

where $\mathbf{C}_{\mathbf{h}_{k}}=\mathbb{E} \mathbf{h}_{k} \mathbf{h}_{k}^{H}$ and

$$
\mathbf{T}=\sqrt{P^{\mathrm{ul}}}[\mathbf{I}, \ldots, \mathbf{I}]^{T}
$$

[27]. The error of this estimation can be models as

$$
\mathbf{h}_{k}=\hat{\mathbf{h}}_{k}+\mathbf{e}_{k}
$$

where $\mathbf{e}_{k}$ is independent of the estimated channel and is Gaussian distributed with covariance given by

$$
\mathbf{C}_{e}=\mathbf{C}_{\mathbf{h}_{k}}-\mathbf{C}_{\mathbf{h}_{k}} \mathbf{T}^{T}\left(\mathbf{T C}_{\mathbf{h}_{k}} \mathbf{T}+\sigma_{n}^{2} \mathbf{I}\right)^{-1} \mathbf{T} \mathbf{C}_{\mathbf{h}_{k}}
$$

As a special case note that if $\mathbf{C}_{\mathbf{h}_{k}}=\sigma_{k}^{2} \mathbf{I}$, the covariance matrix of error is given by

$$
\mathbf{C}_{e}=\sigma_{e}^{2} \mathbf{I}
$$

where $\sigma_{e}^{2}=\frac{\sigma_{n}^{2}}{\sigma_{n}^{2}+L P^{\mathrm{ul}}}$.

\subsubsection{Results for Imperfect CSI}

We assume that the CSI is obtained through reverse channel estimation in TDD using the strategy described in Section 3.3.1, such that the users transmit the pilots with power $P_{\mathrm{ul}}$ one by one, and the base station estimates the channel by using equation (3.10). Figure 


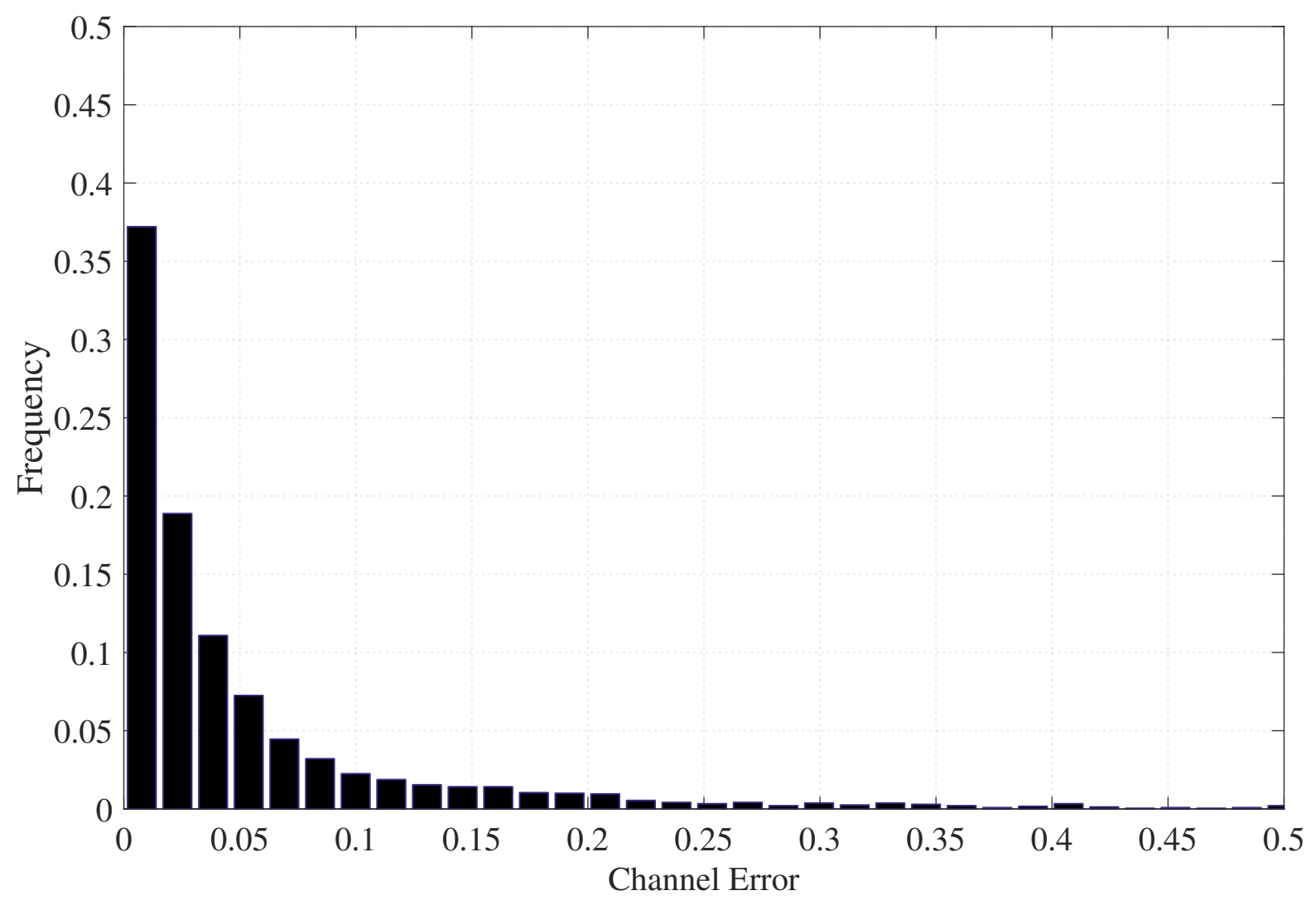

Figure 3.5: Histogram of channels' error

3.5 shows the histogram of

$$
\frac{\left|e_{k}^{n}\right|}{\left|h_{k}^{n}\right|} \quad \forall k, n
$$

where $h_{k}^{n}$ is the channel of user $k$ from antenna $n$ of the base station and $e_{k}^{n}$ is the corresponding estimation error.

Our network is composed of $4 \mathrm{SPs}$, each serving 15 users. The channels are modelled as in (3.1). The InP has a base station equipped with 100 antennas and communicates the CSI of all antennas to the SPs. Other system parameters are presented in Table 3.3.

Figure 3.6 displays the norm of $\mathbf{V}$ with respect to $\rho$ for different values of $N$. Several observations are now in order. The most important one is that the proposed solution is close to the lower bound, described in Section 2.3.2, which indeed means the proposed solution is nearly optimal. We can also observe that as the value of $\rho$ decreases, the norm of $\mathbf{V}$ increases until a point at which the problem becomes infeasible.

Figure 3.7 shows the same curves but with respect to $N$. In this figure, we observe that more antennas can benefit the InP by giving a feasible solution for smaller value of 


\begin{tabular}{|l|c|}
\hline Parameter & Value \\
\hline$M$ & 4 \\
\hline$K_{m}$ & 15 \\
\hline$N$ & 100 \\
\hline$N_{m}$ & 100 \\
\hline$P_{\mathrm{ul}}$ & $20 \mathrm{dBm}$ \\
\hline$\rho$ & 0.1 \\
\hline$\epsilon$ & 0.1 \\
\hline $\mathrm{L}$ & 5 \\
\hline
\end{tabular}

Table 3.3: Default simulation parameters for imperfect CSI

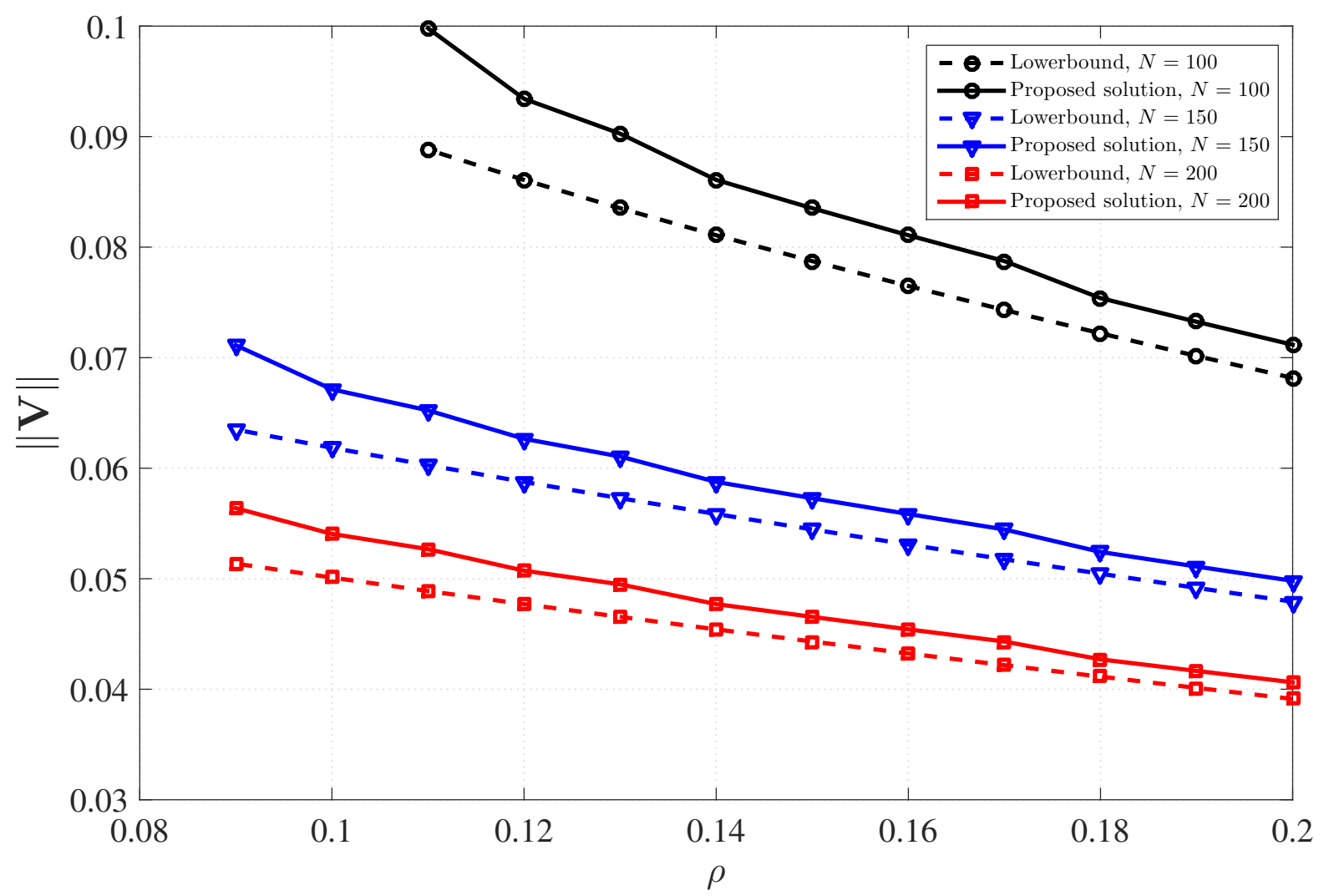

Figure 3.6: Norm of $\mathbf{V}^{*}$ vs $\rho$ for imperfect CSI 


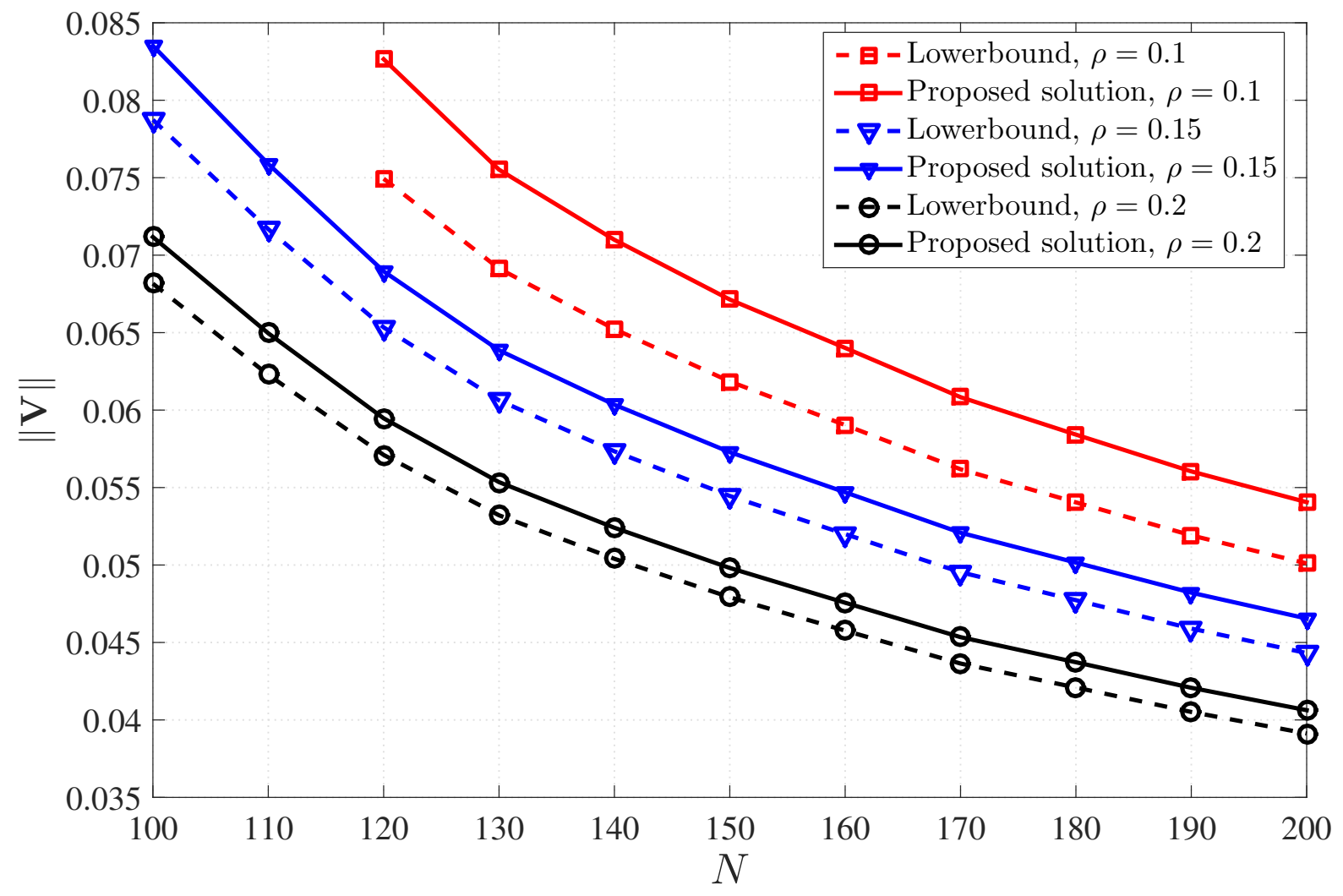

Figure 3.7: Norm of $\mathbf{V}^{*}$ vs $N$ for imperfect CSI

$\rho$. This phenomenon can somewhat encourage the InP to have more antennas than the ones' it provides to the SPs to be able satisfies the SP's expectations and find a feasible solution.

Figure 3.8 demonstrates the maximum channel error that the problem is feasible for different values of $\rho$. It also depicts an upper-bound for the feasible solutions. This figure asserts that our proposed algorithm almost always provides a solution when there is a feasible solution.

Finally in Figure 3.9, we verifies the assumptions we have made to propose the lowerbound. This figure plots the CDF of (2.11) and compares it with the CDF of the normal distribution. As one can see in this figure, the two curves nearly overlap, which shows the the assumption is reasonable.

\subsection{Three Extensions}

In this section, we report simulation results for the extensions described in 2.4. We assume CSI are perfectly known and study the algorithm proposed in Section 2.2 to conduct the simulations. Notice that we could have also simulated for non-perfect CSI 


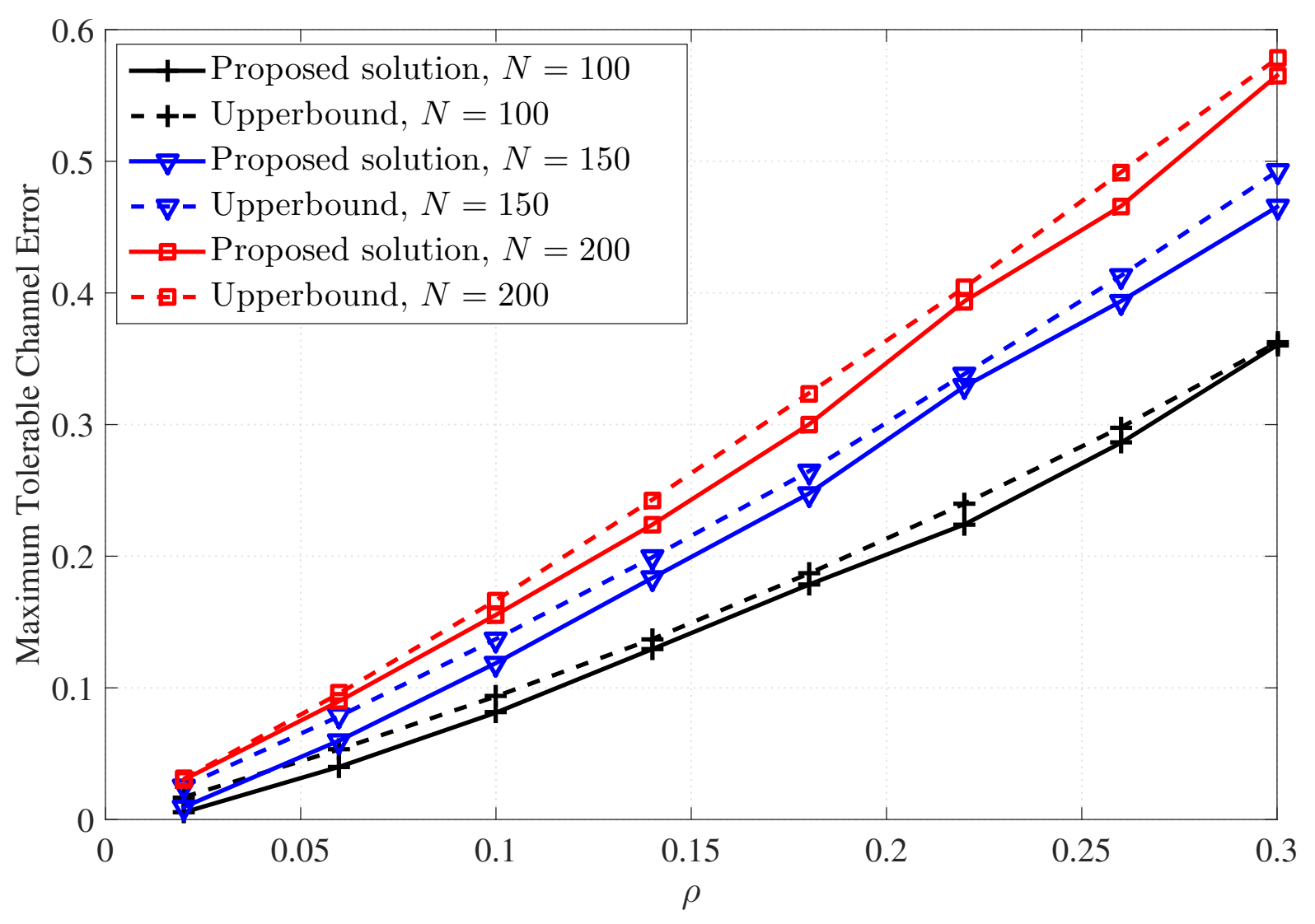

Figure 3.8: Maximum tolerable channel error for a given $\rho$ 


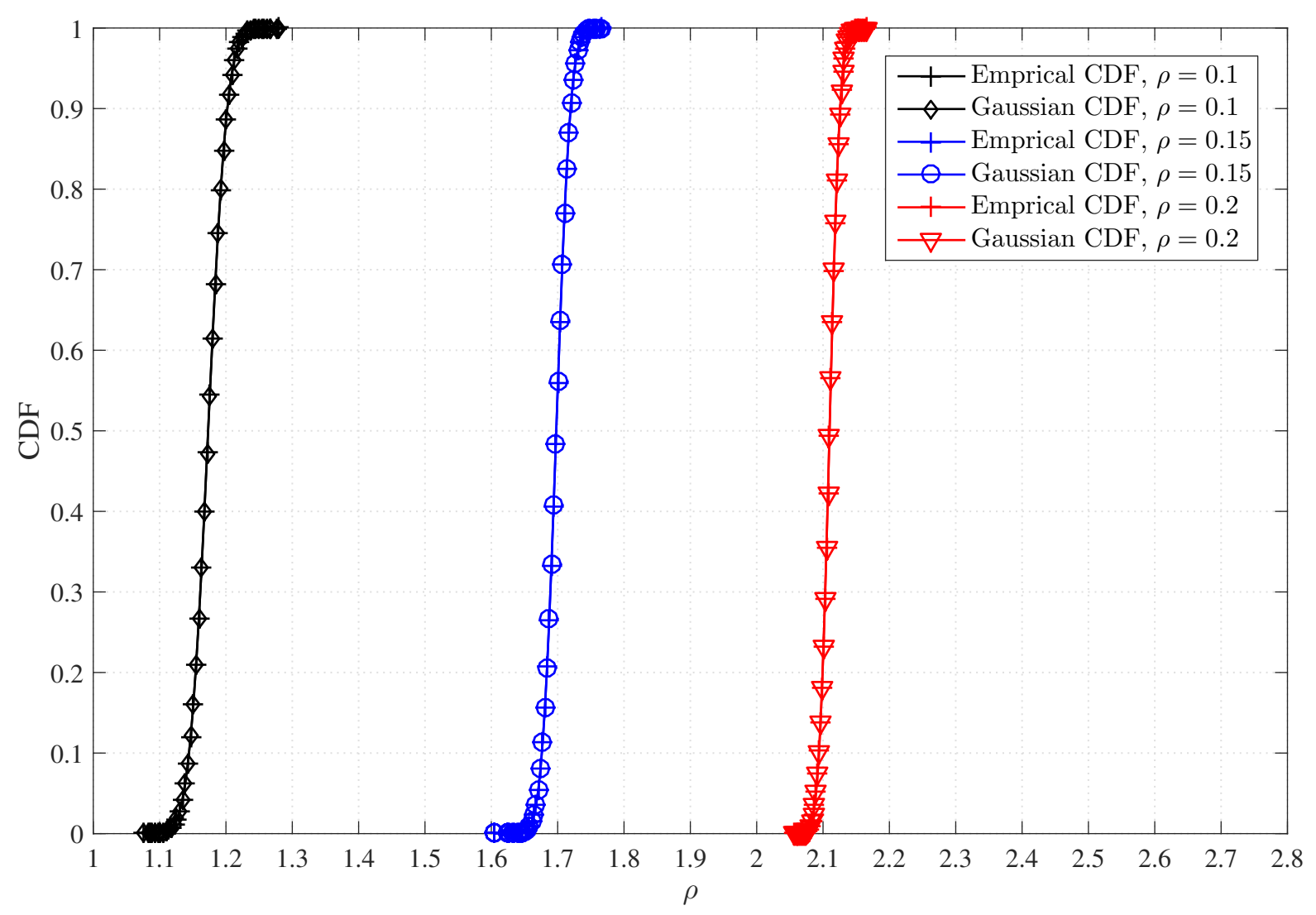

Figure 3.9: CDF of deviation 


\begin{tabular}{|l|c|}
\hline Parameter & Value \\
\hline$M$ & 3 \\
\hline$K_{m}$ & 15 \\
\hline$N$ & 140 \\
\hline$N_{m}$ & 50 \\
\hline
\end{tabular}

Table 3.4: Default simulation parameters for multicell simulation

using the algorithm proposed in Section 2.3.

As mentioned in 2.4.2, we can extend our model to include multi-cell networks by limiting the interference caused in the neighbouring cells. We consider a network composed of 7 cells and 3 SPs, each has 15 users(Figure 3.10 and Table 3.4). In addition to satisfying the SPs performance expectations (constraints 2.11), each base station limits the interference it causes to the closest $K^{\prime}$ users in the neighbouring cells. In Figure 3.11, the received SINR of the users in cell 2 is displayed, for different values of $K^{\prime}$. As can be seen in this figure, mitigating inter-cell interference of the users can favourably impact the users' received signal. However, we observe that when $K^{\prime}$ grows larger, it negatively impacts the received signal. One reason for this observation is that as $K^{\prime}$ increases, more transmission power is required to satisfy the constraints (according to Figure 3.12), and more transmission power means more interference to non-targeted users. The optimal value of $K^{\prime}$ can be potentially a compelling problem to be further considered. Figure 3.12 plots the required transmission power to mitigate the interference to the $K^{\prime}$ closest users in addition to satisfying the SPs constraints.

We now turn to the extension described in 2.4.3 which adds per user constraints to the optimization problem. We use the parameters presented in Table 3.5. To reduce the number of variables, we assume that the SPs request per user constraints for all their users with $\rho_{k}^{m}=\rho$. Figure 3.13.c shows the received SINR of the users of SPs. For the sake of comparison, we also report the received signal designed by the SPs, the receive signal without considering per user constraints in Figures 3.13.a, 3.13.b respectively. As can be seen in this figure, some users' received signals degrade significantly if per user constraints are dismissed. This improvement in the users' received signals is at the price of more transmission power. Figures 3.14 and 3.15 plot the required transmission power for different $\rho$ and $N$ and compare it with the solution without considering per users constraints. 


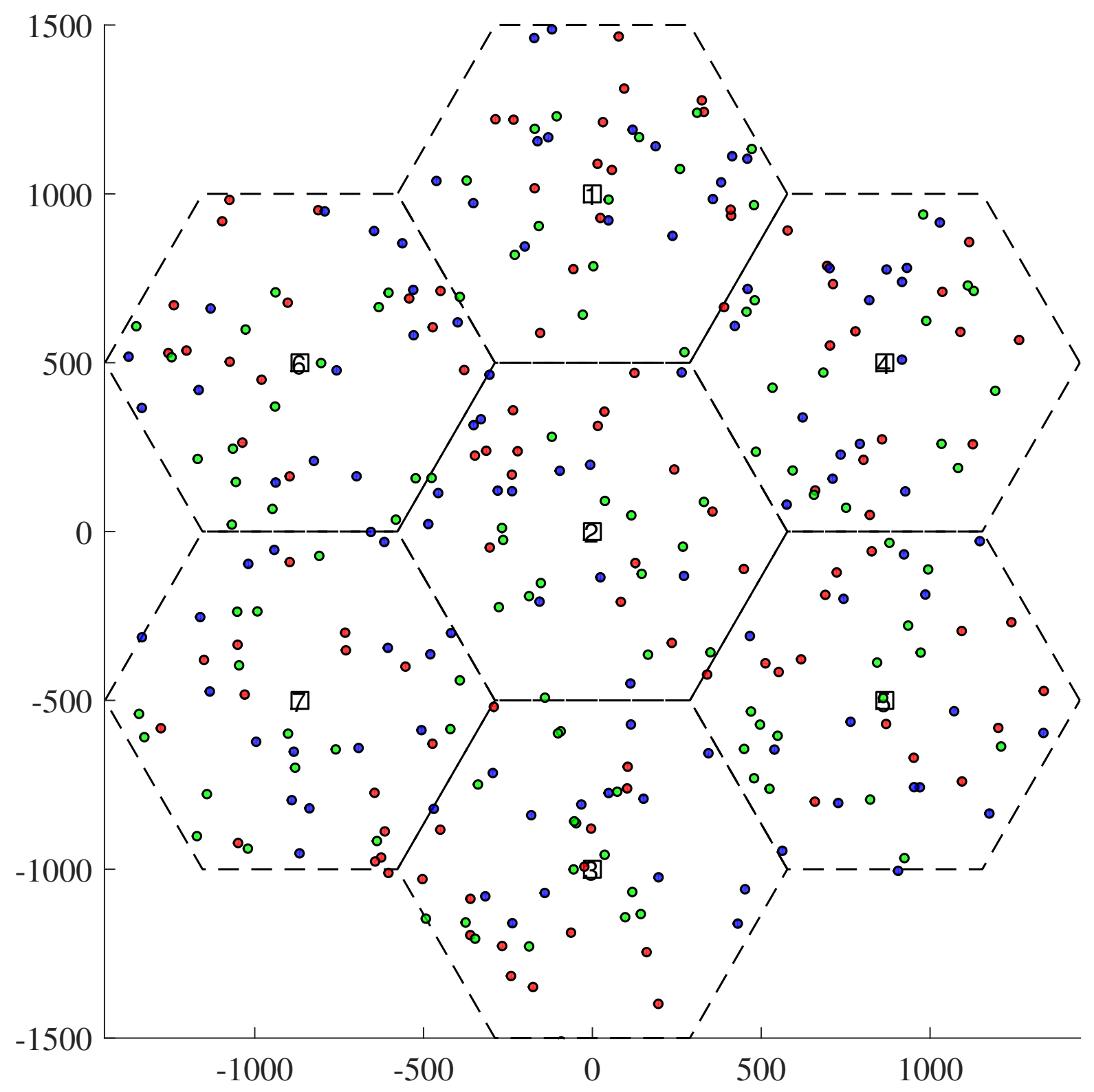

Figure 3.10: A realization of the multi-cell network under consideration. Different colours represent users of different SPS

\begin{tabular}{|l|c|}
\hline Parameter & Value \\
\hline$M$ & 4 \\
\hline$K_{m}$ & 20 \\
\hline$N$ & 140 \\
\hline$N_{m}$ & 40 \\
\hline$P_{\mathrm{ul}}$ & $20 \mathrm{dBm}$ \\
\hline
\end{tabular}

Table 3.5: Default simulation parameters for per-user problem simulation 


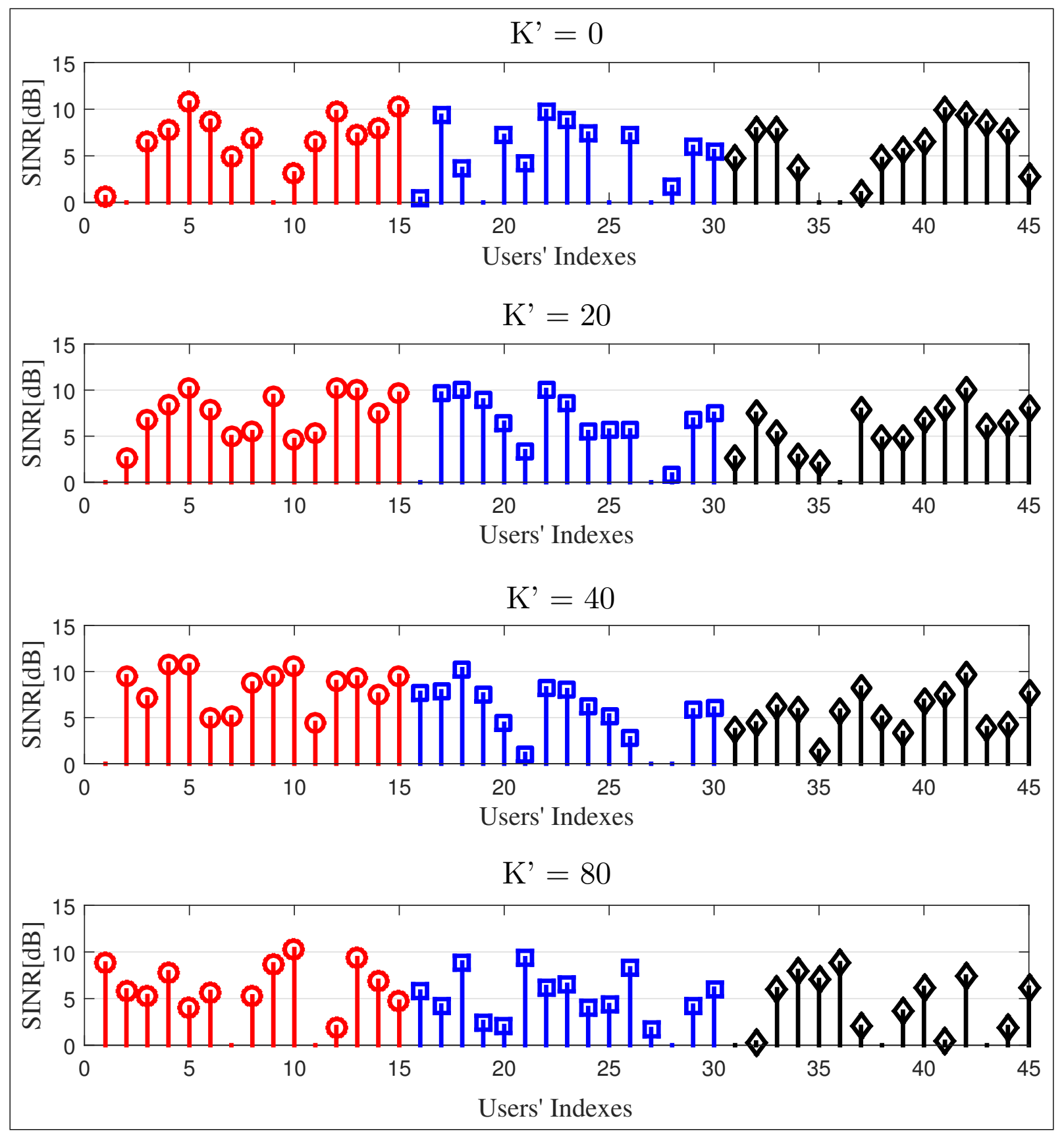

Figure 3.11: Received SINR of users in the multi-cell network for different values of $K^{\prime}$ 


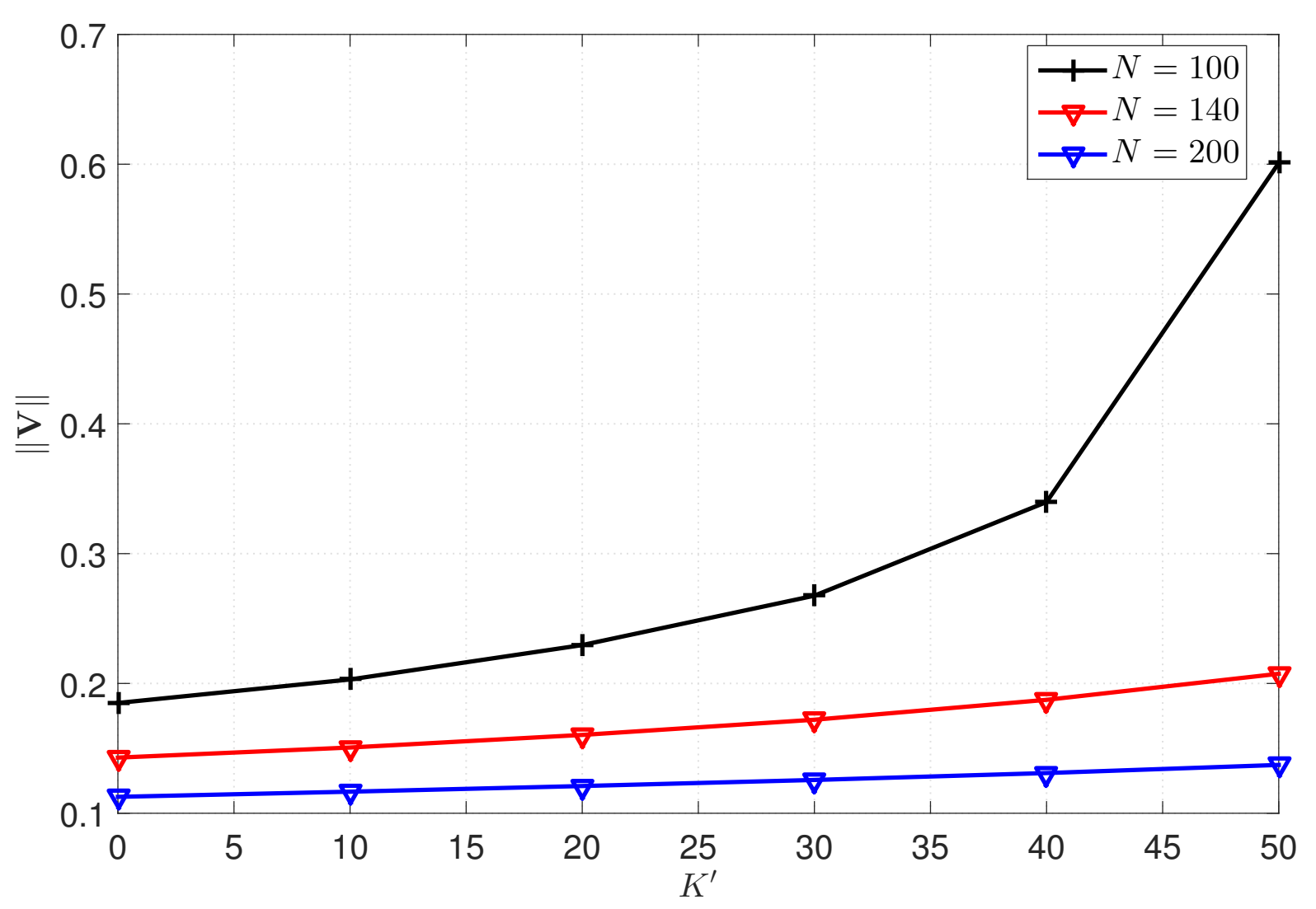

Figure 3.12: Required transmission power vs $K^{\prime}$ 


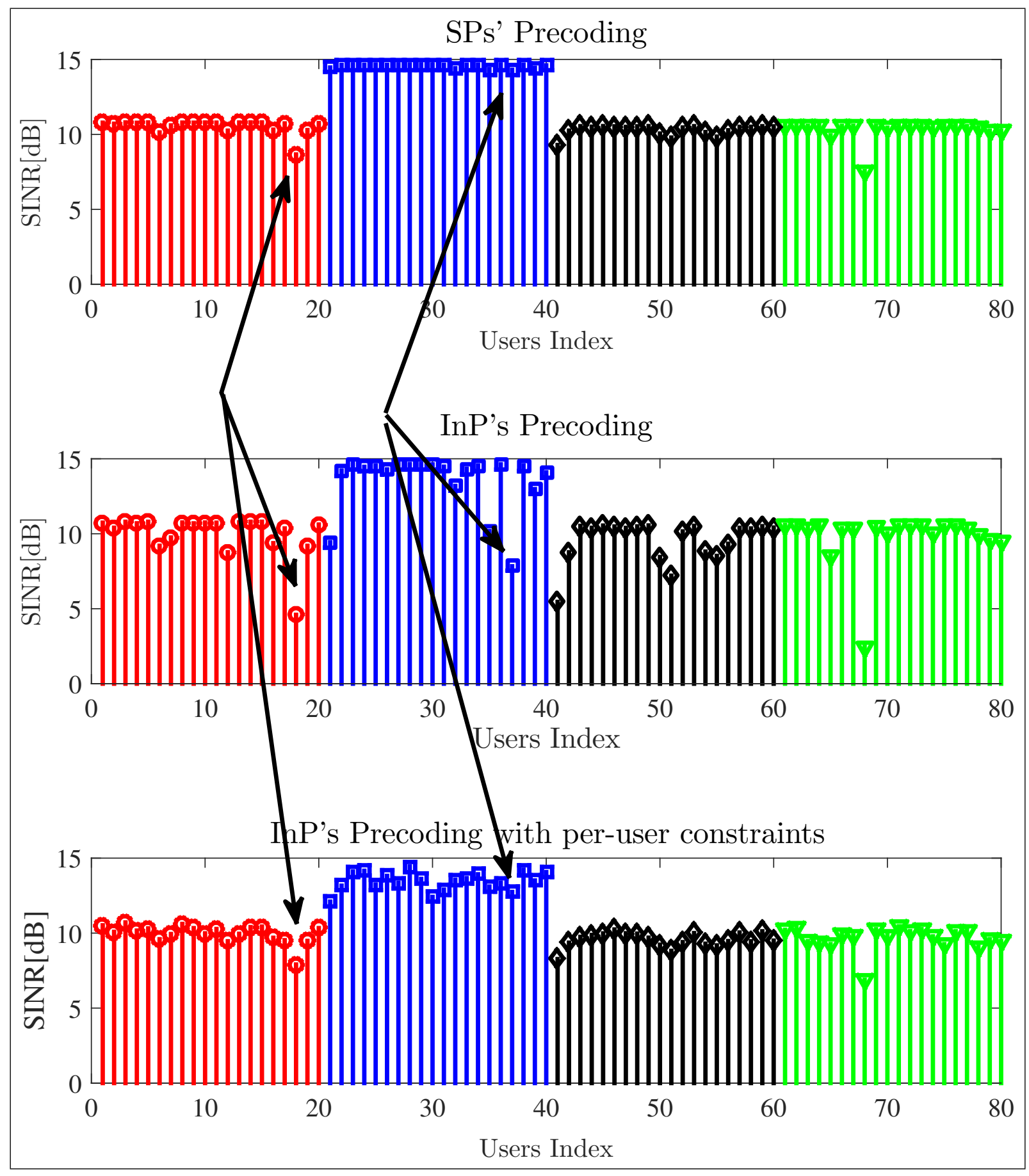

Figure 3.13: Received SINR of the users for different schemes 


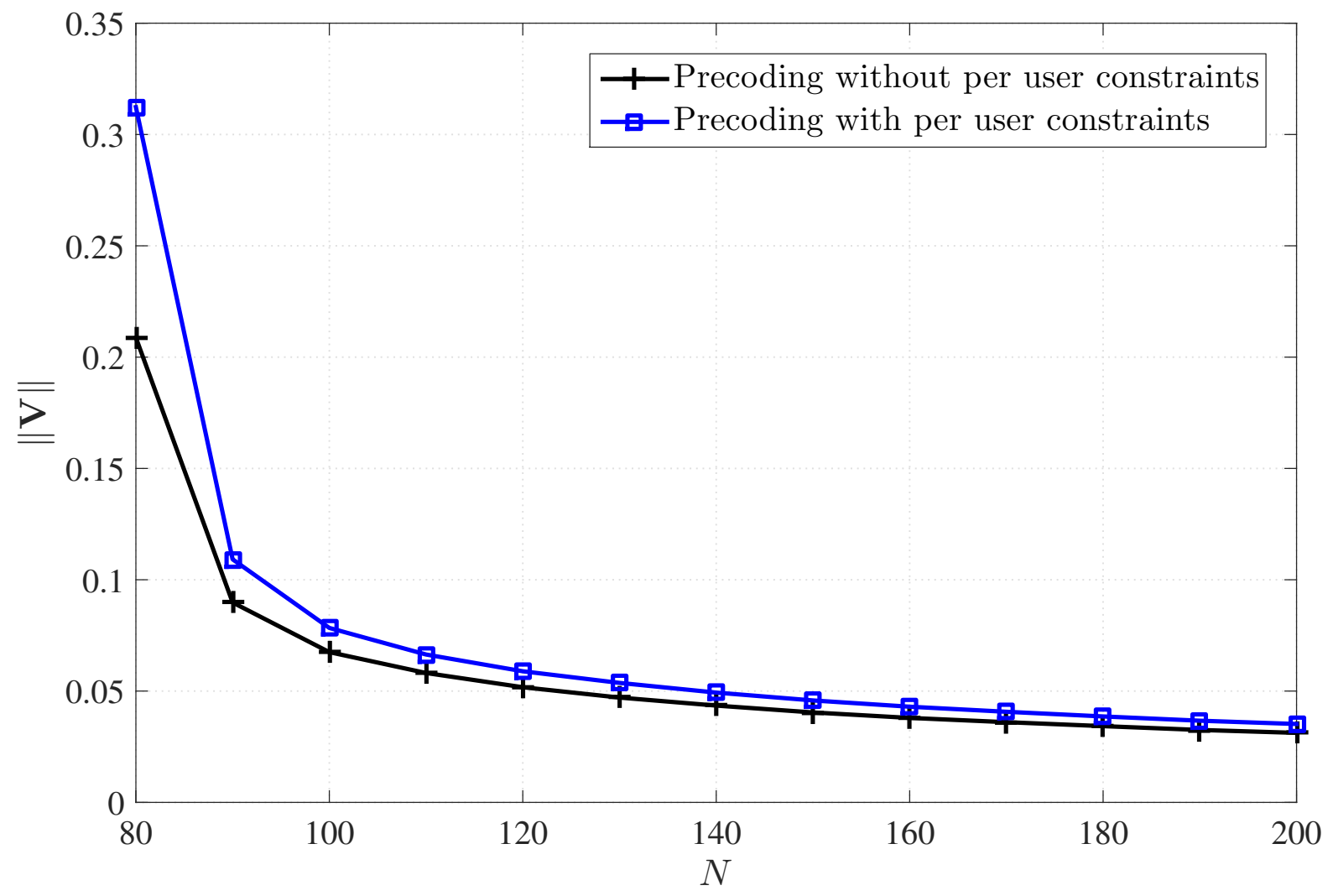

Figure 3.14: Comparison of solutions with per-user constrain solution 


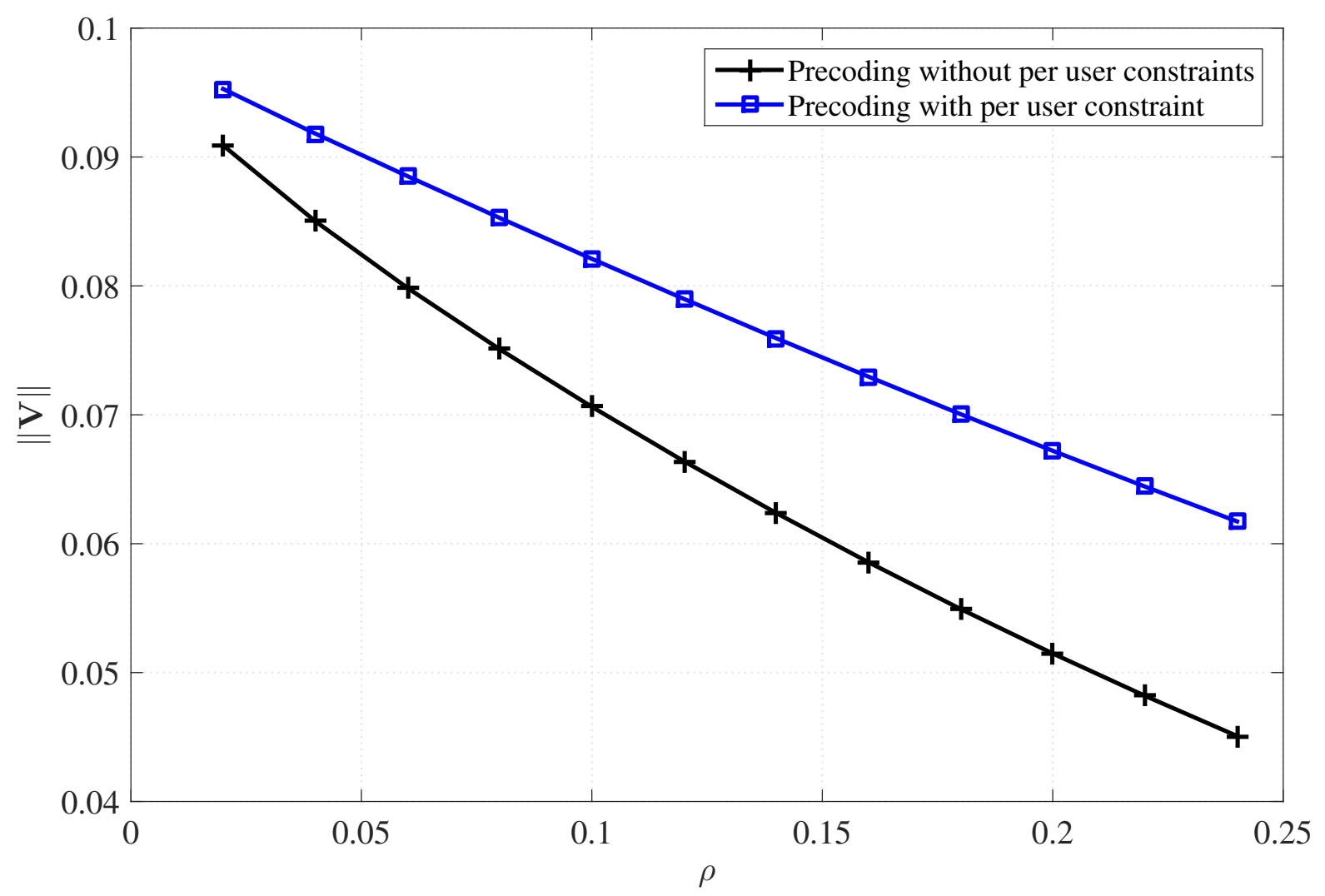

Figure 3.15: Comparison of solutions with per-user constrain solution 


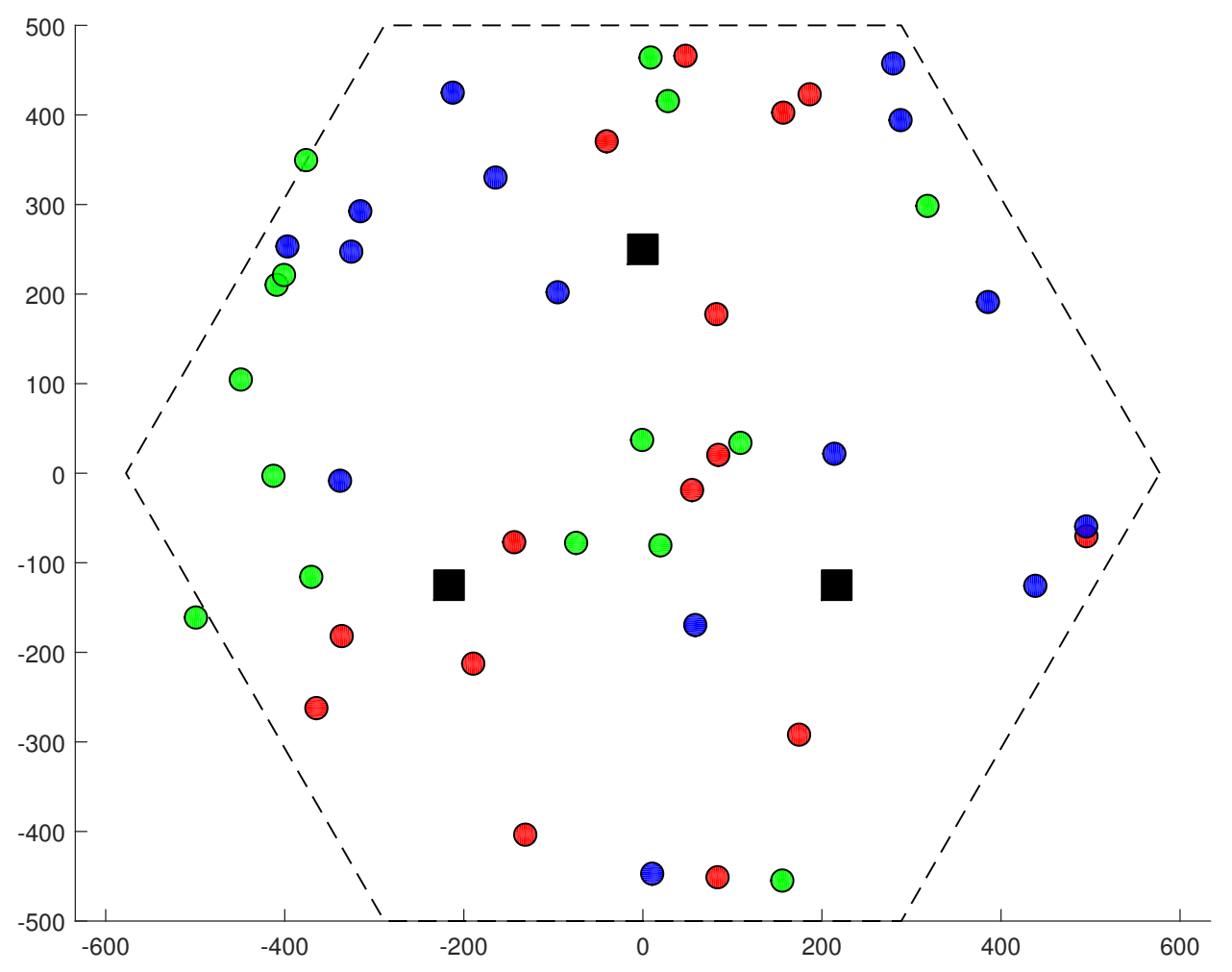

Figure 3.16: A realization of a cell with a BS with distributed antennas. The black squares represent the radio heads.

\subsection{Antenna Selection Algorithms}

We now study the performances of the algorithms proposed for antenna selection associated to Step 1 and Step 3.

First, we conduct simulation for antenna selection in Step 1, i.e. selecting $N_{m}$ antennas for a given SP. In this simulation, we divide the antennas of the BS into three radio heads each having 40 antennas, and they are placed at three different geographical points, as shown in Figure 3.16. This configuration signifies the importance of antenna selection and serves as an example to see the improvement of performance through antenna selection. In Figure 3.17, we plot the total rate of the system when the InP selects antennas based on the following strategies:

- Strategy 1: Scheme proposed in Section 2.5.1

- Strategy 2: Random selection uniformly among all antennas

- Strategy 3: Selecting $N_{m}$ antennas having the maximum average power over the users of the SP $m$. 


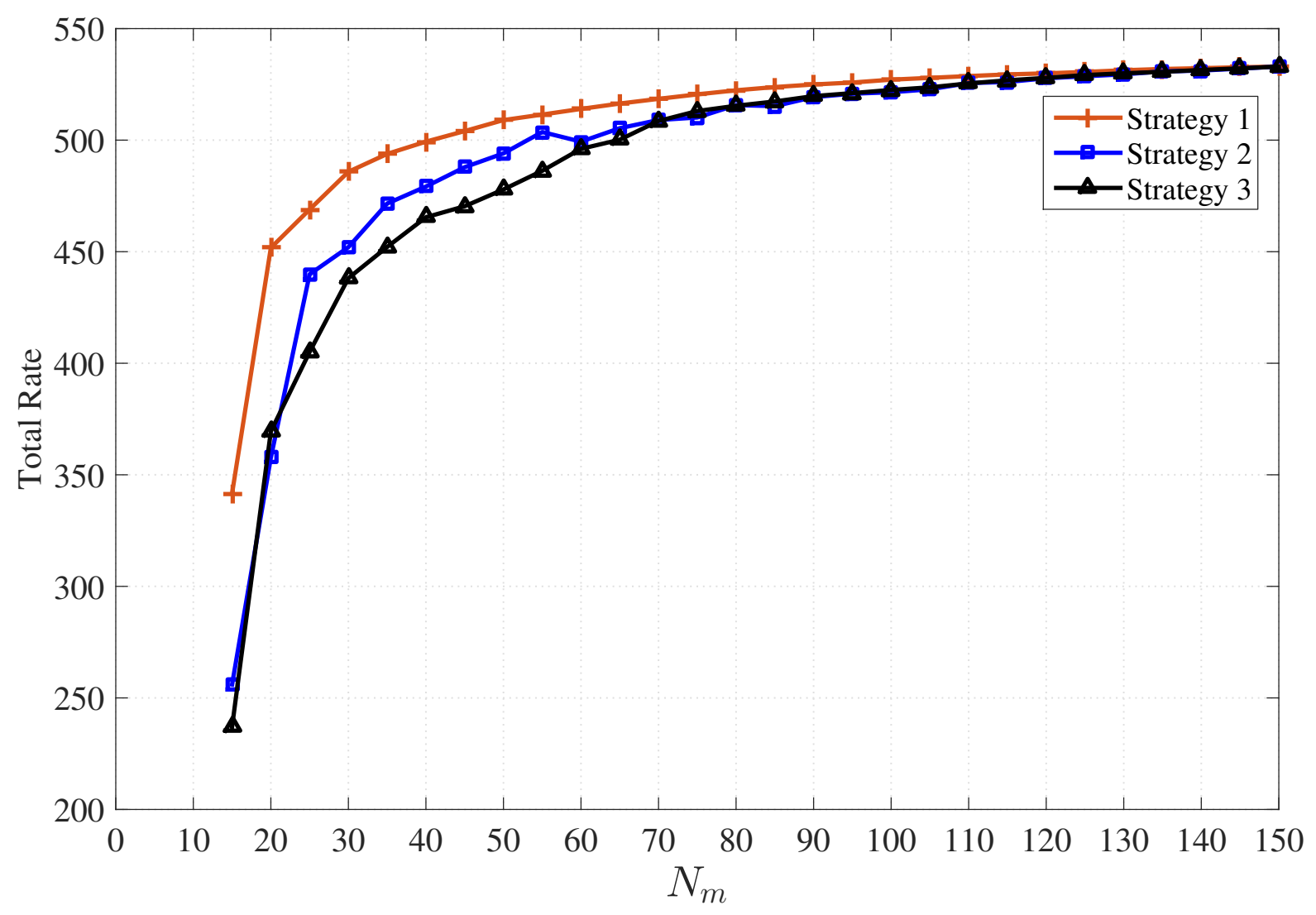

Figure 3.17: Comparison of different strategies of antenna selection 


\begin{tabular}{|l|c|}
\hline Parameter & Value \\
\hline$M$ & 4 \\
\hline$K_{m}$ & 15 \\
\hline$N$ & 120 \\
\hline$N_{m}$ & 40 \\
\hline$\rho$ & 0.05 \\
\hline
\end{tabular}

Table 3.6: Default simulation parameters for antenna selection algorithms in Step 1

\begin{tabular}{|l|c|}
\hline Parameter & Value \\
\hline$M$ & 4 \\
\hline$K_{m}$ & 20 \\
\hline$N$ & 150 \\
\hline$N_{m}$ & 40 \\
\hline$\rho$ & 0.1 \\
\hline Antenna Efficiency & 0.3 \\
\hline$P_{\mathrm{RF}}$ & Power-Ratio $* \mathrm{P}$ \\
\hline
\end{tabular}

Table 3.7: Default simulation parameters for antenna selection algorithms in Step 3

As can be seen in Figure 3.17, for small $N_{m}$, better selection algorithm can considerably improve the overall system performance, particularly when $N_{m} \ll N$.

Second, we conduct simulation for antenna selection in Step 3, i.e. selecting transmission antennas such that total power, including power consumed in RF-circuits, is minimized. The default parameters of the network are presented in Table 3.7. Figure 3.19 reports the optimal number of antennas derived from the algorithm given in 2.5.2, and Figure 3.18 shows the power saved. Performances are parametrized versus power ratio, which is defined as

$$
\text { Power ratio }=\frac{P_{\mathrm{RF}}}{P}
$$

where $P_{\mathrm{RF}}$ is the consumption power per RF-circuit and $P$ is the total power dedicated to the SPs. As this figure shows, the proposed algorithm can save significant amount of the power when the power ratio grows large.

Note that in figure 3.18, parameter $P_{T}$ is defined as follows.

$$
P_{T}=\frac{P\|\mathbf{V}\|^{2}}{\text { Antenna Efficiency }}+P_{\mathrm{RF}} N \text {, }
$$

$P_{N 0}$ is the total power $\left(P_{T}\right)$ when the InP employs all the antennas, and $P_{\text {Nopt }}$ is the total power $\left(P_{T}\right)$ when the InP employs the antennas resulted from the selection algorithm 


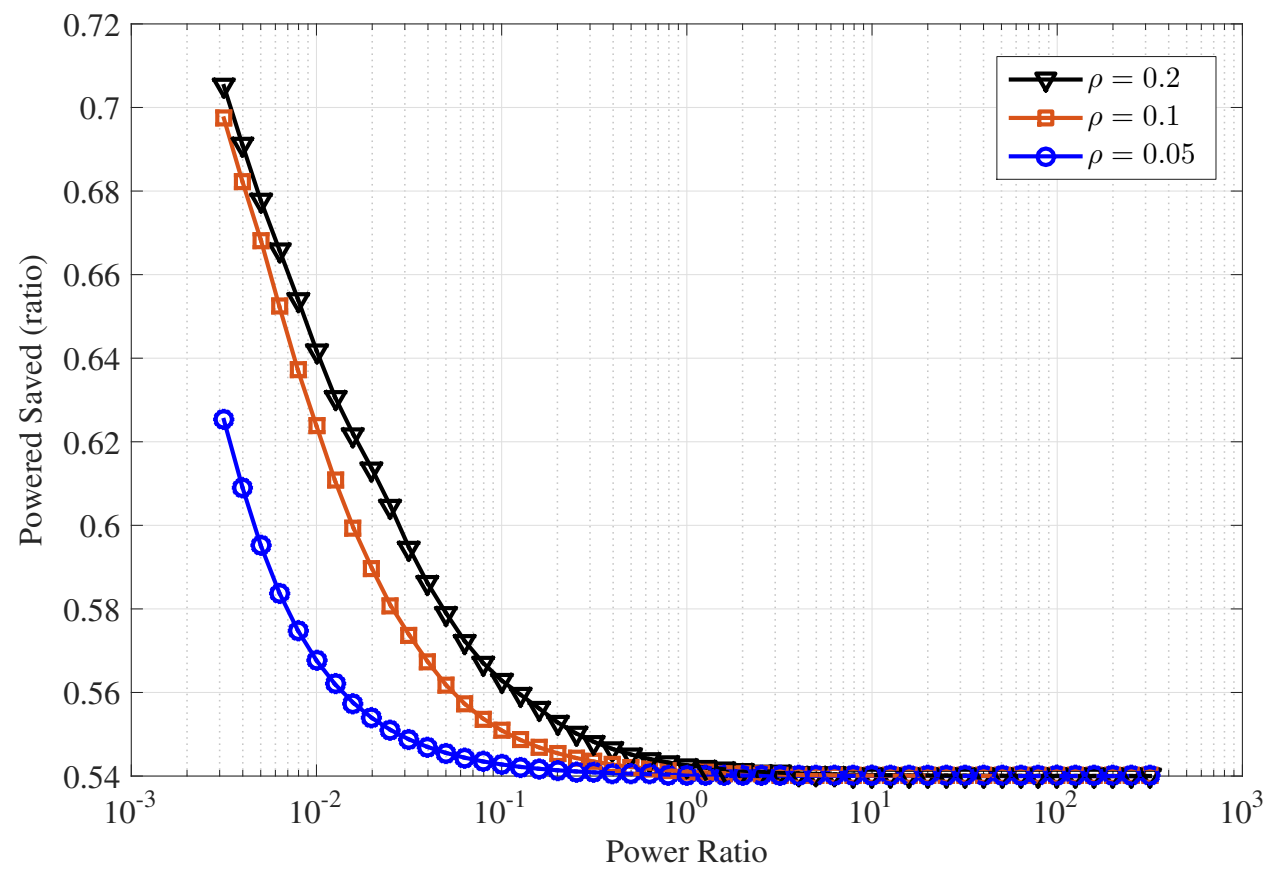

Figure 3.18: Power saved vs different normalized $P_{\mathrm{RF}}$

described above. 


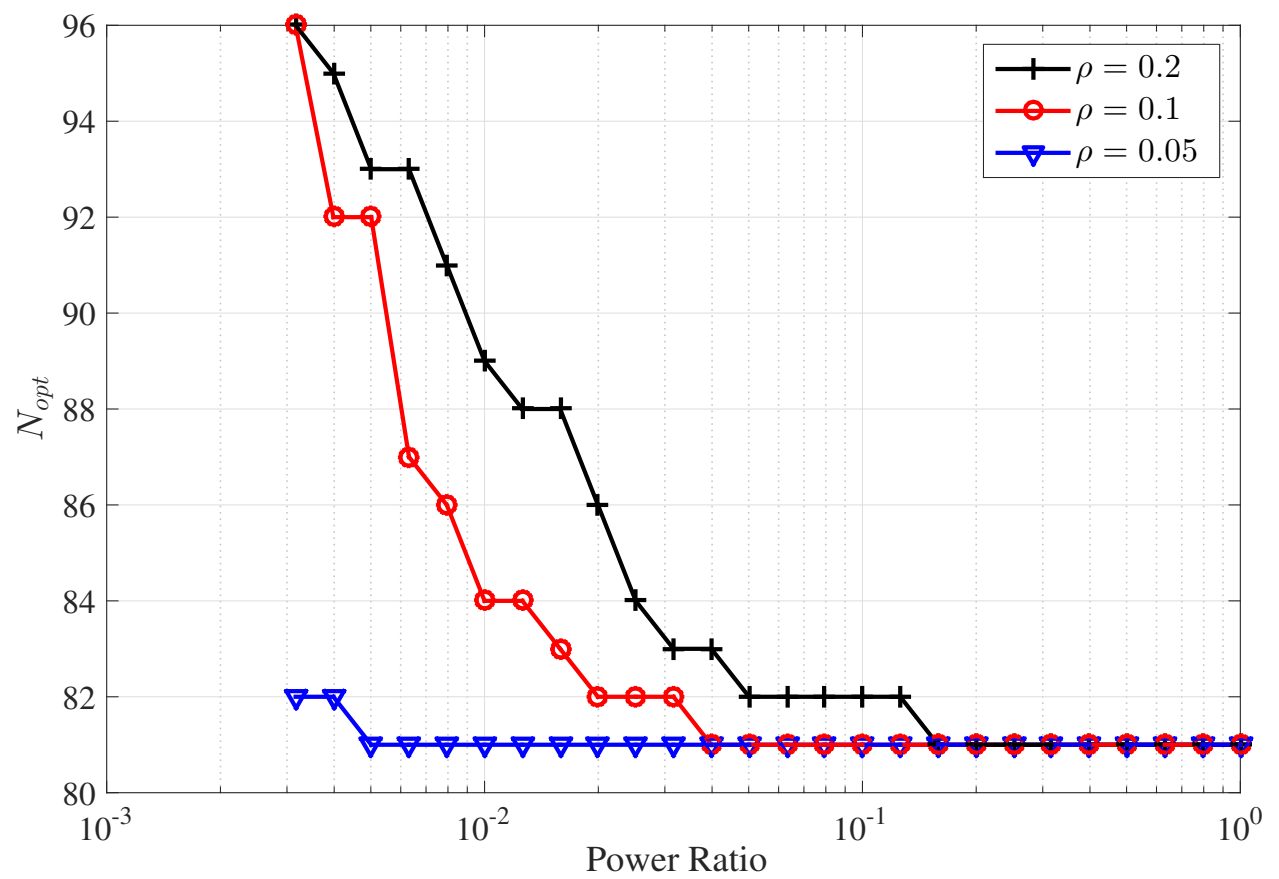

Figure 3.19: Optimal number of antennas derived from the antennas selection algorithm 


\section{Chapter 4}

\section{Conclusion}

\subsection{Contributions}

In this thesis, we investigated virtualization in wireless networks with the massive MIMO technology. We started by reviewing wireless network virtualization, and described progress that has been made to accomplish virtualization in wireless networks. Massive MIMO is considered as a promising technology for $5 \mathrm{G}$ and we proposed a framework for performing virtualization on massive MIMO. To the best our knowledge, this is the first work proposes a formal framework for virtualization of massive MIMO that takes into accounts all the requirements of virtualization.

We formulated the precoding problem in wireless network virtualization when the perfect CSI is available and proposed a simple iterative algorithm to find the optimal precoding with respect to minimum transmission power. We have extended this precoding problem for non-perfect CSI and formulated it with chance constraints. Although the problems with chance constraints are generally intractable, we have proposed a simple and effective solution, and showed that its performance is close to the optimal by providing a lower bound for the optimal solution. We have developed methods to select transmission antennas, and by numerical results showed the effectiveness of the proposed antennas selection algorithms in saving power. We have formulated the virtualization of massive MIMO as general as possible so it also addresses some other important concerns. We showed how this formulation can be extended to incorporate multi-cell networks. Also, we have explained how stricter constraints for the users of the SPs can be included in our formulation. 


\subsection{Future Research Direction}

We have made the assumption that the network composed of only one InP while in reality more then one InPs usually exists in the networks. Studying the problem of multiple InPs can be compelling both from SPs and InPs' perspective. Our model facilitates this extension for multi InPs by providing an approach to limit inter-cell interference. However, other technical issues such as resource allocation, privacy and user scheduling by SPs should be further studied.

We have focused on precoding at the base station with the assumption that the SPs can arbitrarily schedule any number of users. Therefore, there is a possibility that the total number of users exceeds the number of antennas, and as such, the problem becomes infeasible. The InP therefore should limit the number of serving users of the SPs in order to avoid this possibility. Finding a fair and efficient procedure to schedule the SPs is worth studying as it not only should ensure the fairness but also efficient resource allocation. 


\section{Appendix A}

\section{Proofs}

In this appendix we provide the proofs and derivation of the expressions we have referred to throughout the thesis.

\section{A.1 Proof of Lemma 2.2.1}

Proof. To perfectly null inter-SP interference, the equality

$$
\mathbf{H}_{m} \mathbf{V} \mathbf{x}=\mathbf{G}_{m} \mathbf{W}_{m} \mathbf{x}_{m} \quad \forall m
$$

should be satisfied for all SPs. If we re-write these $M$ equations as

$$
\mathbf{H V x}=\mathbf{B x}
$$

where

$$
\begin{aligned}
& \mathbf{H}=\left[\mathbf{H}_{1} ; \ldots, \mathbf{H}_{M}\right] \in \\
& \mathbf{B}=\operatorname{diag}\left(\mathbf{G}_{1} \mathbf{W}_{1}, \ldots, \mathbf{G}_{M} \mathbf{W}_{M}\right)
\end{aligned}
$$

where diag refers to a block diagonal matrix. It suffices that the following equality holds:

$$
\mathrm{HV}=\mathrm{B},
$$

which is an under-determined equation and has infinitely many solutions. Denote $V^{*}$ as the solution to this problem. Then, we have

$$
\left\|\mathbf{H}_{m} \mathbf{V} \mathbf{x}-\mathbf{G}_{m} \mathbf{W}_{m} \mathbf{x}_{m}\right\|^{2}=0 \leq \mathcal{I}_{m} \quad \forall m
$$


Therefore, $\mathbf{V}^{*}$ is a feasible solution to the problem.

Now we turn to the second statement of the lemma and assume that the statement is not true and there is a constraint that is not active, e.g. $m$. Let $\mathbf{V}^{*}=\left[\mathbf{V}_{1}^{*}, \ldots, \mathbf{V}_{M}^{*}\right]$, $\mathbf{V}_{m} \neq \mathbf{0}$ be the optimal solution to the problem. Then the following inequality holds:

$$
\left\|\mathbf{H}_{m} \mathbf{V}_{m}^{*}-\mathbf{G}_{m} \mathbf{W}_{m}\right\|^{2}+\sum_{i \neq m}\left\|\mathbf{H}_{m} \mathbf{V}_{i}^{*}\right\|^{2}<\mathcal{I}_{m}^{2}
$$

Define $f(\alpha)$ as follows:

$$
f(\alpha)=\mathcal{I}_{m}^{2}-\left\|\mathbf{H}_{m}\left(\alpha \mathbf{V}_{m}^{*}\right)-\mathbf{G}_{m} \mathbf{W}_{m}\right\|^{2}-\sum_{i \neq m}\left\|\mathbf{H}_{m} \mathbf{V}_{i}^{*}\right\|^{2},
$$

We have

$$
\begin{aligned}
& f(1)>0 \\
& f(0)<0
\end{aligned}
$$

since $f(\alpha)$ is a continues function. According to the mean value theorem, there is a $0<\alpha^{*}<1$ such that

$$
f\left(\alpha^{*}\right)=0 .
$$

Now, define $\mathbf{V}^{\prime}$ as follows:

$$
\mathbf{V}^{\prime}=\left[\mathbf{V}_{1}, \ldots, \alpha^{*} \mathbf{V}_{m}, \ldots, \mathbf{V}_{M}\right]
$$

We have

$$
\left\|\mathbf{H}_{i} \mathbf{V}_{m}^{\prime}\right\|<\left\|\mathbf{H}_{i} \mathbf{V}^{*}\right\| \quad \forall i \neq m .
$$

Hence

$$
\begin{aligned}
&\left\|\mathbf{H}_{i} \mathbf{V}_{i}^{\prime}-\mathbf{G}_{i} \mathbf{W}_{i}\right\|^{2}+\sum_{j \neq i}\left\|\mathbf{H}_{i} \mathbf{V}_{j}^{\prime}\right\|^{2} \\
&<\left\|\mathbf{H}_{i} \mathbf{V}_{i}^{*}-\mathbf{G}_{i} \mathbf{W}_{i}\right\|^{2}+\sum_{j \neq i}\left\|\mathbf{H}_{i} \mathbf{V}_{j}^{*}\right\|^{2} \leq \mathcal{I}_{m} \quad \forall i \neq m,
\end{aligned}
$$


and also from (A.11)

$$
\left\|\mathbf{H}_{m} \mathbf{V}_{m}^{\prime}-\mathbf{G}_{m} \mathbf{W}_{m}\right\|^{2}+\sum_{i \neq m}\left\|\mathbf{H}_{m} \mathbf{V}_{i}^{\prime}\right\|^{2}=\mathcal{I}_{m}^{2}
$$

Therefore $\mathbf{V}^{\prime}$ is a feasible solution. However, since $\alpha<1$, we have the following inequality

$$
\left\|\mathbf{V}^{\prime}\right\|<\left\|\mathbf{V}^{*}\right\|
$$

which is a contradiction with $\mathbf{V}^{*}$ being the minimum norm feasible solution. Therefore, all the constraint should be active.

\section{A.2 Proof of Lemma 2.3.1}

Proof. We can assume $\mathbf{e} \sim \mathcal{C N}\left(\mathbf{0}, \mathbf{I}_{M}\right)$ by re-defining $\mathbf{A}$ as follows:

$$
\mathbf{A}_{\text {new }}=\mathbf{A}_{\text {old }} \mathbf{C}_{e} .
$$

By definition, the reliability probability is obtained by

$$
\mathbb{P}\left(\|\mathbf{A e}+\mathbf{b}\|^{2} \leq \tau\right)=\int_{-\infty}^{\infty} p(\mathbf{e}) u\left(\tau-\|\mathbf{A} \mathbf{e}+\mathbf{b}\|^{2}\right) d \mathbf{e}
$$

where $p($.$) is the PDF of \mathbf{e}$ and $u($.$) is the unit step function. Therefore$

$$
\mathbb{P}\left(\|\mathbf{A e}+\mathbf{b}\|^{2} \leq \tau\right)=\frac{1}{\pi^{M}} \int_{-\infty}^{\infty} e^{-\|\mathbf{e}\|^{2}} u\left(\tau-\|\mathbf{A} \mathbf{e}-\mathbf{b}\|^{2}\right) d \mathbf{e} .
$$

The Fourier transform of the unit step is obtained by

$$
u(z)=\frac{1}{2 \pi} \int_{-\infty}^{\infty} \frac{e^{z(i \omega+\beta)}}{i \omega+\beta} d \omega
$$


for any $\beta>0$. By substituting this transform into (A.20) and reordering integrals, we have

$$
\begin{aligned}
& \mathbb{P}\left(\|\mathbf{A e}+\mathbf{b}\|^{2} \leq \tau\right) \\
= & \frac{1}{2 \pi^{M+1}} \int_{-\infty}^{\infty} \frac{e^{\tau(i \omega+\beta)}}{i \omega+\beta} \int_{-\infty}^{\infty} e^{-\left(\|\mathbf{e}\|^{2}+(i \omega+\beta)\|\mathbf{A} \mathbf{e}+\mathbf{b}\|^{2}\right)} d \mathbf{e} d \omega \\
= & \frac{1}{2 \pi^{M+1}} \int_{-\infty}^{\infty} \frac{e^{\tau(i \omega+\beta)}}{i \omega+\beta} \int_{-\infty}^{\infty} e^{-f(\mathbf{e})} d \mathbf{e} d \omega
\end{aligned}
$$

where

$$
\begin{aligned}
& f(\mathbf{e})=\|\mathbf{e}\|^{2}+s\|\mathbf{A e}+\mathbf{b}\|^{2} \\
& s=i \omega+\beta .
\end{aligned}
$$

Let $\mathbf{U D Q}=\operatorname{svd}(\mathbf{A})$. We can simplify $f(\mathbf{e})$ as follows:

$$
\begin{aligned}
f(\mathbf{e}) & =\mathbf{e}^{H} \mathbf{e}+s \mathbf{e}^{H} \mathbf{A}^{H} \mathbf{A e}+s \mathbf{e}^{H} \mathbf{A}^{H} \mathbf{b}+s \mathbf{b}^{H} \mathbf{A e}+s \mathbf{b}^{H} \mathbf{b} \\
& =\mathbf{e}^{H} \mathbf{e}+s \mathbf{e}^{H} \mathbf{Q}^{H} \mathbf{D}^{2} \mathbf{Q e}+s \mathbf{e}^{H} \mathbf{Q}^{H} \mathbf{D} \mathbf{U}^{H} \mathbf{b}+s \mathbf{b}^{H} \mathbf{U D Q} \mathbf{e}+s \mathbf{b}^{H} \mathbf{b} \\
& =\mathbf{e}^{H}\left(\mathbf{I}+s \mathbf{Q}^{H} \mathbf{D}^{2} \mathbf{Q}\right) \mathbf{e}+s \mathbf{e}^{H} \mathbf{Q}^{H} \mathbf{D} \mathbf{U}^{H} \mathbf{b}+s \mathbf{b}^{H} \mathbf{U D Q}+s \mathbf{b}^{H} \mathbf{b} \\
& =\mathbf{e}^{H} \mathbf{Q}^{H}\left(\mathbf{I}+s \mathbf{D}^{2}\right) \mathbf{Q e}+s \mathbf{e}^{H} \mathbf{Q}^{H} \mathbf{D} \mathbf{U}^{H} \mathbf{b}+s \mathbf{b}^{H} \mathbf{U D Q}+s \mathbf{b}^{H} \mathbf{b} \\
& =\mathbf{e}_{1}^{H}\left(\mathbf{I}+s \mathbf{D}^{2}\right) \mathbf{e}_{1}+s \mathbf{e}_{1}^{H} \mathbf{D a}+s \mathbf{a}^{H} \mathbf{D} \mathbf{e}_{1}+s \mathbf{b}^{H} \mathbf{b}
\end{aligned}
$$

where

$$
\begin{aligned}
& \mathbf{e}^{\prime}=\mathbf{Q e} \\
& \mathbf{a}=\mathbf{U}^{H} \mathbf{b}
\end{aligned}
$$

Notice that since $\mathbf{Q}$ is a unitary matrix, we have

$$
d \mathbf{e}^{\prime}=|\operatorname{det}(\mathbf{Q})| d \mathbf{e}=d \mathbf{e},
$$

and also $\mathbf{e}^{\prime}$ is distributed as $\mathcal{C N}(\mathbf{0}, \mathbf{I})$.

From (A.31), we have

$$
f(\mathbf{e})=\sum_{m}\left(1+s D_{m}^{2}\right)\left|e_{m}^{\prime}\right|^{2}+s D_{m}\left(e_{m}^{* *} a_{m}+e_{m}^{\prime} a_{m}^{*}\right)+s \mathbf{b}^{H} \mathbf{b}
$$

where $e_{m}^{\prime}$ and $a_{m}$ are the $m$ th elements of vectors $\mathbf{e}^{\prime}$ and a respectively. 
By substituting (A.35) into (A.24), we obtain

$$
\begin{aligned}
& \mathbb{P}\left(\|\mathbf{A} \mathbf{e}+\mathbf{b}\|^{2} \leq \tau\right) \\
& =\frac{1}{2 \pi^{M+1}} \int_{-\infty}^{\infty} \frac{e^{\tau(i \omega+\beta)}}{i \omega+\beta} e^{-s \mathbf{b}^{H} \mathbf{b}} \prod_{m} \int_{-\infty}^{\infty} e^{-\left[\left(1+s D_{m}^{2}\right)\left|e_{m}^{\prime}\right|^{2}+s D_{m}\left(e_{m}^{\prime *} a_{m}+e_{m}^{\prime} a_{m}^{*}\right)\right]} d e_{m}^{\prime} d \omega .
\end{aligned}
$$

We decompose the above integral over $e_{m}^{\prime}$ into two integrals, one over $x_{m}=\operatorname{Re}\left\{e_{m}^{\prime}\right\}$ and one over $y_{m}=\operatorname{Im}\left\{e_{m}^{\prime}\right\}$, and then by completing the squares in the exponent we have

$$
\begin{aligned}
& \int_{-\infty}^{\infty} e^{-\left[\left(1+s D_{m}^{2}\right)\left|e_{m}^{\prime}\right|^{2}+s D_{m}\left(e_{m}^{\prime *} a_{m}+e_{m}^{\prime} a_{m}^{*}\right)\right]} d e_{m}^{\prime} \\
= & e^{\frac{s^{2} D_{m}^{2}\left|a_{m}\right|^{2}}{1+s D_{m}^{2}}} \int_{-\infty}^{\infty} e^{-\left(1+s D_{m}^{2}\right)\left(x_{m}-\frac{s D_{m} \operatorname{Re}\left\{a_{m}\right\}}{1+s D_{m}^{2}}\right)} d x_{m} \int_{-\infty}^{\infty} e^{-\left(1+s D_{m}^{2}\right)\left(y_{m}-\frac{s D_{m} \operatorname{Im}\left\{a_{m}\right\}}{1+s D_{m}^{2}}\right)} d y_{m}
\end{aligned}
$$

If we choose $\operatorname{Re}\{s\}=\beta>0$, this integral can be simplified to

$$
\begin{aligned}
& \int_{-\infty}^{\infty} e^{-\left[\left(1+s D_{m}^{2}\right)\left|e_{m}^{\prime}\right|^{2}+s D_{m}\left(e_{m}^{\prime *} a_{m}+e_{m}^{\prime} a_{m}^{*}\right)\right]} d e_{m}^{\prime} \\
= & e^{c_{m}} \frac{\pi^{1 / 2}}{\left(1+s D_{m}^{2}\right)^{1 / 2}} \frac{\pi^{1 / 2}}{\left(1+s D_{m}^{2}\right)^{1 / 2}} \\
= & e^{c_{m}} \frac{\pi}{\left(1+s D_{m}^{2}\right)}
\end{aligned}
$$

By substituting (A.42) into (A.42), we obtain

$$
\begin{aligned}
& \mathbb{P}\left(\|\mathbf{A e}+\mathbf{b}\|^{2} \leq \tau\right) \\
= & \frac{1}{2 \pi^{M+1}} \int_{-\infty}^{\infty} \frac{e^{\tau(i \omega+\beta)}}{i \omega+\beta} e^{-c_{0}} \prod_{m} e^{c_{m}} \frac{\pi}{\left(1+s D_{m}^{2}\right)} d \omega \\
= & \frac{1}{2 \pi} \int_{-\infty}^{\infty} \frac{e^{\tau(i \omega+\beta)}}{i \omega+\beta} \frac{e^{-c_{0}+\sum_{m} c_{m}}}{\prod_{m}\left(1+(i \omega+\beta) D_{m}^{2}\right)} d \omega
\end{aligned}
$$

where

$$
c_{0}=s \mathbf{b}^{H} \mathbf{b} .
$$




\section{A.3 Transforming (2.11) to sum of random variables}

For notation simplicity, we use $\mathbf{W}_{m}$ for $\left[\mathbf{0}, \ldots, \mathbf{W}_{m}, \ldots, \mathbf{0}\right]$ in the following derivations. Therefore, for each $m$ we can transform deviation into vector form as follows:

$$
\begin{aligned}
& \left\|\mathbf{H}_{m} \mathbf{V}-\mathbf{G}_{m} \mathbf{W}_{m}\right\|^{2} \\
= & \left\|\mathbf{E}_{m} \mathbf{V}+\hat{\mathbf{H}}_{m} \mathbf{V}-\mathbf{G}_{m} \mathbf{W}_{m}\right\|^{2} \\
= & \left\|\mathbf{A e}_{m}+\mathbf{b}\right\|^{2}
\end{aligned}
$$

where

$$
\begin{aligned}
& \mathbf{e}_{m}=\operatorname{vec}\left\{\mathbf{E}_{m}\right\} \\
& \mathbf{A}=\mathbf{V}^{T} \otimes \mathbf{I} \\
& \mathbf{b}=\operatorname{vec}\left\{\hat{\mathbf{H}}_{m} \mathbf{V}-\mathbf{G}_{m} \mathbf{W}_{m}\right\} .
\end{aligned}
$$

We note that random vector $\mathbf{e}_{m}$ is distributed as $\mathcal{C N}\left(\mathbf{0}, \mathbf{C}_{e}\right)$. For notational simplicity, for the rest of this section, we redefine $\mathbf{A}$ as

$$
\mathbf{A}=\left(\mathbf{V}^{T} \otimes \mathbf{I}\right) \mathbf{C}_{e}^{1 / 2}
$$

and $\mathbf{e}_{m} \sim \mathcal{C N}\left(\mathbf{0}, \mathbf{I}_{K N}\right)$ then (A.49) still holds.

Assume that the singular value decomposition of $\mathbf{A}$ is given by

$$
[\mathbf{U}, \mathbf{D}, \mathbf{Q}]=\operatorname{svd}(\mathbf{A})
$$

then from (A.49) we have

$$
\begin{aligned}
& \left\|\mathbf{H}_{m} \mathbf{V}-\mathbf{G}_{m} \mathbf{W}_{m}\right\|^{2} \\
= & \left\|\mathbf{A} \mathbf{e}_{m}+\mathbf{b}\right\|^{2} \\
= & \left\|\mathbf{U} \mathbf{D Q} \mathbf{e}_{m}+\mathbf{b}\right\|^{2} \\
= & \left\|\mathbf{D Q} \mathbf{e}_{m}+\mathbf{U}^{H} \mathbf{b}\right\|^{2} \\
= & \left\|\mathbf{D} \mathbf{e}_{m}^{\prime}+\mathbf{a}\right\|^{2}
\end{aligned}
$$


where

$$
\begin{aligned}
& \mathbf{e}_{m}^{\prime}=\mathbf{Q} \\
& \mathbf{a}=\mathbf{U}^{H} \mathbf{b} .
\end{aligned}
$$

Note that since $\mathbf{Q}$ is unitary matrix, we have $\mathbf{e}_{m} \sim \mathcal{C N}\left(\mathbf{0}, \mathbf{I}_{K N}\right)$. Hence,

$$
\begin{aligned}
& \left\|\mathbf{H}_{m} \mathbf{V}-\mathbf{G}_{m} \mathbf{W}_{m}\right\|^{2} \\
= & \left\|\mathbf{D} \mathbf{e}_{m}^{\prime}+\mathbf{a}\right\|^{2} \\
= & \sum_{i=1}^{K N}\left|D_{i} e_{m}^{i}+a_{i}\right|^{2}
\end{aligned}
$$

where $D_{i}$ is the $i$ th element on the diagonal of $\mathbf{D}$ and $\mathbf{e}_{m}^{i}$ and $a_{i}$ are the $i$ th elements of vectors $e_{m}^{\prime}$ and $\mathbf{a}_{m}$ respectively.

\section{A.4 A Property of normally distributed random vari- able}

For a Gaussian random variable $x \sim \mathcal{C}\left(\mu, \sigma^{2}\right)$ we have

$$
\mathbb{P}(x \geq \alpha) \leq \epsilon \equiv \mu \leq \alpha-\sigma \mathrm{Q}^{-1}(\epsilon)
$$

Since

$$
\mathrm{Q}^{-1}(\epsilon) \geq 0
$$

for $\epsilon<0.5$, it follows that

$$
\mathbb{P}(x \geq \alpha) \leq \epsilon \Rightarrow \mu \leq \alpha
$$




\section{A.5 Derivation of the expected value given in (2.56)}

For convenience in notations, we abuse them and redefine $\mathbf{W}_{m}$ as $\mathbf{W}_{m}$ padded with zero. We have

$$
\begin{aligned}
& \mathbb{E}\left\|\mathbf{H}_{m} \mathbf{V}-\mathbf{G}_{m} \mathbf{W}_{m}\right\|^{2} \\
= & \mathbb{E}\left\|\mathbf{E}_{m} \mathbf{V}+\hat{\mathbf{H}}_{m} \mathbf{V}-\mathbf{G}_{m} \mathbf{W}_{m}\right\|^{2} \\
= & \mathbb{E} \operatorname{Trace}\left\{\mathbf{E}_{m} \mathbf{V} \mathbf{V}^{H} \mathbf{E}_{m}^{H}+\mathbf{E}_{m} \mathbf{V}\left(\hat{\mathbf{H}}_{m} \mathbf{V}-\mathbf{G}_{m} \mathbf{W}_{m}\right)^{H}+\right. \\
& \left.\quad \mathbf{V}^{H} \mathbf{E}_{m}^{H}\left(\hat{\mathbf{H}}_{m} \mathbf{V}-\mathbf{G}_{m} \mathbf{W}_{m}\right)\right\}+\left\|\hat{\mathbf{H}}_{m} \mathbf{V}-\mathbf{G}_{m} \mathbf{W}_{m}\right\|^{2} \\
= & \mathbb{E} \operatorname{Trace}\left\{\mathbf{E}_{m} \mathbf{V} \mathbf{V}^{H} \mathbf{E}_{m}^{H}\right\}+\left\|\hat{\mathbf{H}}_{m} \mathbf{V}-\mathbf{G}_{m} \mathbf{W}_{m}\right\|^{2} \\
= & \mathbb{E}\left\|\mathbf{E}_{m} \mathbf{V}\right\|^{2}+\left\|\hat{\mathbf{H}}_{m} \mathbf{V}-\mathbf{G}_{m} \mathbf{W}_{m}\right\|^{2}
\end{aligned}
$$

The first term in (A.72) can be simplified as follows:

$$
\begin{aligned}
& \mathbb{E}\|\mathbf{E V}\|^{2} \\
= & \mathbb{E} \operatorname{Trace}\left\{\mathbf{E V V} \mathbf{E}_{m}^{H} \mathbf{E}^{H}\right\} \\
= & \mathbb{E} \operatorname{Trace}\left\{\mathbf{E}^{H} \mathbf{E V V} \mathbf{V}^{H}\right\} \\
= & \mathbb{E} \sum_{i, j} \mathbf{e}_{j}^{H} \mathbf{e}_{i} \mathbf{v}_{j} \mathbf{v}_{i}^{H}
\end{aligned}
$$

where $e_{j}$ is the $i$ th column of $\mathbf{E}$ and $\mathbf{v}_{j}$ is the $j$ th row of $\mathbf{V}$.

Since

$$
\mathbb{E} \mathbf{e}_{j}^{H} \mathbf{e}_{i}=0 \quad \forall j \neq i
$$


and from (A.76) we have

$$
\begin{aligned}
& \mathbb{E}\|\mathbf{E V}\|^{2} \\
= & \sum_{i} \mathbb{E} \mathbf{e}_{i}^{H} \mathbf{e}_{i} \mathbf{v}_{i} \mathbf{v}_{i}^{H} \\
= & \sum_{i} \mathbb{E} \mathbf{e}_{i}^{H} \mathbf{e}_{i}\left\|\mathbf{v}_{i}\right\|^{2} \\
= & \sum_{i}\left\|\mathbf{v}_{i}\right\|^{2} \mathbb{E} \mathbf{e}_{i}^{H} \mathbf{e}_{i} \\
= & \sum_{i}\left\|\mathbf{v}_{i}\right\|^{2} \mathbb{E} \sum_{j}\left|e_{i j}\right|^{2} \\
= & \sum_{i}\left\|\mathbf{v}_{i}\right\|^{2} \sum_{j} \mathbb{E}\left|e_{i j}\right|^{2} \\
= & \sum_{i}\left\|\mathbf{v}_{i}\right\|^{2} \sum_{j} \sigma_{i j}^{2} \\
= & \sum_{i}\left\|\mathbf{v}_{i}\right\|^{2} D_{i}^{2} \\
= & \sum_{i}\left\|D_{i} \mathbf{v}_{i}\right\|^{2} \\
= & \|\mathbf{D V}\|^{2} .
\end{aligned}
$$




\section{Bibliography}

[1] X. Wang, P. Krishnamurthy, and D. Tipper, "Wireless Network Virtualization," in Proc. International Conference on Computing, Networking and Communications (ICNC), San Diego, CA, Jan 2013.

[2] R. Kokku, R. Mahindra, H. Zhang, and S. Rangarajan, "NVS: a substrate for virtualizing wireless resources in cellular networks," IEEE/ACM Transactions on Networking, vol. 20, pp. 1333-1346, Oct 2012.

[3] C. Liang and F. R. Yu, "Wireless network virtualization: A survey, some research issues and challenges," IEEE Communications Surveys $\&$ Tutorials, vol. 17, no. 1, pp. 358-380, 2015.

[4] G. Schaffrath, C. Werle, P. Papadimitriou, A. Feldmann, R. Bless, A. Greenhalgh, A. Wundsam, M. Kind, O. Maennel, and L. Mathy, "Network virtualization architecture: proposal and initial prototype," in Proc. ACM workshop on Virtualized Infrastructure Systems 83 Architectures, New York,NY, 2009.

[5] R. Bless and C. Werle, "Network virtualization from a signaling perspective," in Proc. IEEE Int. Conf. Commun. (ICC), pp. 1-6, Dresden, Jun 2009.

[6] A. Belbekkouche, M. M. Hasan, and A. Karmouch, "Resource discovery and allocation in network virtualization," IEEE Communications Surveys $\& 3$ Tutorials, vol. 14, pp. 1114-1128, Feb 2012.

[7] T. L. Marzetta, "Noncooperative cellular wireless with unlimited numbers of base station antennas," IEEE Transactions on Wireless Communications, vol. 9, pp. 3590-3600, Nov 2010.

[8] H. Q. Ngo, E. G. Larsson, and T. L. Marzetta, "Energy and spectral efficiency of very large multiuser mimo systems," IEEE Transactions on Communications, vol. 61, pp. 1436-1449, Apr 2013. 
[9] M. Costa, "Writing on dirty paper," IEEE Transactions on Information theory, vol. 29, pp. 439-441, May 1983.

[10] C. B. Peel, B. M. Hochwald, and A. L. Swindlehurst, "A vector-perturbation technique for near-capacity multiantenna multiuser communication-part i: channel inversion and regularization," IEEE Transactions on Communications, vol. 53, pp. 195202, Jan 2005.

[11] C. Windpassinger, R. F. Fischer, and J. B. Huber, "Lattice-reduction-aided broadcast precoding," IEEE Transactions on Communications, vol. 52, pp. 2057-2060, Dec 2004.

[12] F. Rusek, D. Persson, B. K. Lau, E. G. Larsson, T. L. Marzetta, O. Edfors, and F. Tufvesson, "Scaling up mimo: Opportunities and challenges with very large arrays," IEEE Signal Processing Magazine, vol. 30, pp. 40-60, Jan 2013.

[13] Y. Zhou, Y. Li, G. Sun, D. Jin, L. Su, and L. Zeng, "Game theory based bandwidth allocation scheme for network virtualization," in Proc. IEEE Global Telecommunications Conference (Globecom), pp. 1-5, IEEE, Dec 2010.

[14] M. Yang, Y. Li, L. Zeng, D. Jin, and L. Su, "Karnaugh-map like online embedding algorithm of wireless virtualization," in Proc. International Symposium on Wireless Personal Multimedia Communications, Taipei, Taiwan, Sept 2012.

[15] X. Zhang, Y. Li, D. Jin, L. Su, L. Zeng, and P. Hui, "Efficient resource allocation for wireless virtualization using time-space division," in Proc.International Wireless Communications and Mobile Computing Conference (IWCMC), Aug 2012.

[16] F. Fu and U. C. Kozat, "Wireless network virtualization as a sequential auction game," in Proc. IEEE INFOCOM, San Diego, CA, Mar 2010.

[17] T. K. Forde, I. Macaluso, and L. E. Doyle, "Exclusive sharing \& virtualization of the cellular network," in Proc. IEEE Symp. on New Frontiers in Dynamic Spectrum Access Net. (DySPAN), Aachen, May 2011.

[18] Y. Zaki, L. Zhao, C. Goerg, and A. Timm-Giel, "A novel LTE wireless virtualization framework," in Proc. ICST Conference on Mobile Networks And Management, Santander, Spain, Sep 2010.

[19] Y. Zaki, L. Zhao, C. Goerg, and A. Timm-Giel, "LTE wireless virtualization and spectrum management," in Proc. IFIP Wireless and Mobile Networking Conference $(W M N C)$, Budapest, Hungary, October 2010. 
[20] Y. Zaki, L. Zhao, C. Goerg, and A. Timm-Giel, "LTE Mobile Network Virtualization," Mobile Networks and Applications, vol. 16, no. 4, pp. 424-432, 2011.

[21] A. Banchs, P. Serrano, P. Patras, and M. Natkaniec, "Providing throughput and fairness guarantees in virtualized WLANs through control theory," Mobile Networks and Applications, vol. 17, no. 4, pp. 435-446, 2012.

[22] G. Bhanage, D. Vete, I. Seskar, and D. Raychaudhuri, "SplitAP: leveraging wireless network virtualization for flexible sharing of WLANs," in Proc. IEEE Global Telecommunications Conference (Globecom), Miami, FL, Dec 2010.

[23] D. Yun and Y. Yi, "Virtual network embedding in wireless multihop networks," in Proc. Intl Conf. Future Internet Technologies, Seul, Jun 2011.

[24] V. Jumba, S. Parsaeefard, M. Derakhshani, and T. Le-Ngoc, "Resource provisioning in wireless virtualized networks via massive-mimo," IEEE Wireless Communications Letters, vol. 4, pp. 237-240, June 2015.

[25] H. Yang and T. L. Marzetta, "Performance of conjugate and zero-forcing beamforming in large-scale antenna systems," IEEE Journal on Selected Areas in Communications, vol. 31, no. 2, pp. 172-179, 2013.

[26] T. Y. Al-Naffouri and B. Hassibi, "On the distribution of indefinite quadratic forms in gaussian random variables," in Proc. Int. Symp. Info. Theory, pp. 1744-1748, Seul, 2009.

[27] H. V. Poor, An introduction to signal detection and estimation. Springer Science \& Business Media, 2013. 